\title{
The paleoinclination of the ancient lunar magnetic field from an Apollo 17 basalt
}

Claire Nichols ( $\square$ claire.nichols@earth.ox.ac.uk)

University of Oxford https://orcid.org/0000-0003-2947-5694

\section{Benjamin Weiss}

MIT

Brenna Getzin

MIT

Harrison Schmitt

University of Wisconsin-Madison

Annemarieke Beguin

Utrecht University

Auriol Rae

University of Freiburg

Jay Shah

MIT

\section{Research Article}

Keywords: lunar magnetic field, paleomagnetic studies, Apollo 17 basalt

Posted Date: March 16th, 2021

DOI: https://doi.org/10.21203/rs.3.rs-311626/v1

License: (1) (1) This work is licensed under a Creative Commons Attribution 4.0 International License. Read Full License

Version of Record: A version of this preprint was published at Nature Astronomy on September 27th, 2021. See the published version at https://doi.org/10.1038/s41550-021-01469-y. 


\section{The Paleoinclination of the Ancient Lunar}

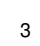
777 Massachusetts Avenue, Cambridge, MA 02139, USA

$8^{b}$ Now at: Department of Earth Sciences, University of Oxford, South Parks Road, Oxford, OX1 3AN,

9 UK

Claire I. O. Nichols ${ }^{a, b *}$, Benjamin P. Weiss ${ }^{a}$, Brenna L. Getzin ${ }^{a}$, Harrison H. Schmitt ${ }^{c}$, Annemarieke Béguin $^{d}$, Auriol S.P. Rae ${ }^{e, f}$, Jay Shah $^{a}$

*Corresponding author: claire.nichols@earth.ox.ac.uk

${ }^{a}$ Department of Earth, Atmospheric and Planetary Sciences, Massachusetts Institute of Technology,

${ }^{c}$ Department of Engineering Physics, University of Wisconsin-Madison, P.O. Box 90730, Albuquerque, NM 87199-0730, USA

${ }^{d}$ Paleomagnetic laboratory Fort Hoofddijk, Department of Earth Sciences, Utrecht University, Budapestlaan 17, 3584 CD Utrecht, The Netherlands

${ }^{e}$ Institute of Earth and Environmental Sciences - Geology, Albert-Ludwigs Universiät Freiburg, Albertstrasse 23b, 79104 Freiburg, Germany

${ }^{f}$ Department of Earth Sciences, University of Cambridge, Downing Street, Cambridge, CB2 3EQ, UK

Paleomagnetic studies of Apollo samples indicate that the Moon generated a core dynamo lasting for at least 2 billion years ${ }^{1,2,3}$. However, the geometry of the lunar magnetic field is still largely unknown because the original orientations of essentially all Apollo samples have not been well-constrained. Determining the direction of the lunar magnetic field over time could elucidate the mechanism by which the lunar dynamo was powered and whether the Moon experienced true polar wander. Here we present measurements of the lunar magnetic field 3.7 billion years $(\mathrm{Ga})$ ago as recorded by Apollo 17 mare basalts 75035 and 75055. These samples formed as part of basalt flows in the Taurus-Littrow valley that make up wall-rock within Camelot crater, now exposed at the rim of the crater ${ }^{4}$. Using apparent layering in the parent boulder for 75055 , we inferred

\section{Magnetic Field from an Apollo 17 Basalt}


its original paleohorizontal orientation on the lunar surface at the time of magnetization. We find that 75035 and 75055 record a mean paleointensity of $\sim 50 \mu \mathrm{T}$. Furthermore, 75055 records a paleoinclination of $34 \pm 11^{\circ}$. This inclination is consistent with, but does not require, a selenocentric axial dipole field geometry (i.e., a dipole in the center of the Moon and aligned along the spin axis). Additionally, although true polar wander is also not required by our data, true polar wander paths inferred from some independent studies of lunar hydrogen deposits and crustal magnetic anomalies ${ }^{5,6,7}$ are consistent with our measured paleoinclination.

Measurements of the paleodirection of the Earth's field have played a fundamental role in our understanding of the geodynamo and the history of global tectonics ${ }^{8}$. These studies have shown that the time-averaged surface magnetic field [over at least several million years (Ma)] of the Earth is largely indistinguishable from that of a geocentric axial dipole. The relationship between magnetic inclination, $I$, and latitude, $\lambda$, of an axial dipolar magnetic field is given by

$$
\tan I=2 \tan \lambda
$$

For any given latitude, Equation (1) predicts the expected mean paleoinclination of an Earth-like, dynamo field. Applications of this relationship to constrain the paleolatitude of rocks laid the foundation for the discovery of plate tectonics. Likewise, lunar paleodirectional data would enable a test of whether the Moon's dynamo was described by an equivalent selenocentric axial dipole relationship and to assess the implications for the dynamo mechanism and the possibility of true polar wander.

However, unambiguous constraints on lunar paleomagnetism have almost exclusivity been limited to paleointensity data. These measurements indicate that the early lunar dynamo generated surface fields possibly reaching $\sim 70 \mu \mathrm{T}$ or more from $4.25-3.56$ Ga ago. The lunar dynamo field subsequently declined to $5 \pm 2 \mu \mathrm{T}$ before apparently ceasing sometime between 1.92 and $0.80 \mathrm{Ga} \mathrm{ago}^{2,3}$. By comparison, only two studies of Apollo samples have attempted to constrain the absolute paleodirection of the lunar magnetic field ${ }^{5,9}$. One study of mare basalts tentatively suggested that the lunar dynamo $3.3-$ $3.8 \mathrm{Ga}$ ago was dipolar and aligned along the present-day spin axis ${ }^{5}$. The other study ${ }^{9}$ was conducted on regolith breccia 15015, but the stable magnetization in this sample has subsequently been shown to have been produced during saw cutting at the Johnson Space Center (JSC) ${ }^{3}$. Both studies attempted 
to infer the field's paleoinclination using magnetic anisotropy measurements, but the uncertainties on the paleohorizontal orientation of the samples from data are difficult to quantify. In particular, our analyses of mare basalts find magnetic anisotropy to be a poor indicator of paleohorizontality (see Supplementary Text S2 and Figure S15).

Numerous studies have attempted to constrain the geometry of the ancient magnetic field using spacecraft measurements of the crustal magnetic field. Although some such studies found support for the presence of a selenocentric axial dipole geometry from the clustering of some paleopoles, the paleopoles as a whole are spread over the entire surface of the Moon ${ }^{6,10}$. The large scatter of the paleopoles is likely in part a consequence of the fact that, even for a uniformly magnetized source with a single component of magnetization, inverted paleopoles depend on assumptions about the source, depth and susceptibility of magnetization, resulting in nonunique magnetization solutions ${ }^{11,12}$. Furthermore, if the crust has multiple components of magnetization acquired at different times throughout lunar history, then even if a paleopole could be uniquely recovered from the net magnetization, it would not correspond to the paleopoles associated with any of the composite magnetization events. Finally, it is difficult to determine the age of paleopoles inferred from orbital data given the uncertainty of using crater counting ages to date deep crustal magnetization sources and the lack of direct radiometric constraints. Therefore, measuring the paleomagnetic directions recorded by lunar samples whose original orientations can be reconstructed, and which can be dated with radiometric techniques, is likely the most unambiguous way to reconstruct lunar paleopoles.

The relative contributions of an axial dipole and multipolar terms to a dynamo field are thought to be influenced by the local Rossby number, $R o_{l}$, which quantifies the ratio of inertial to Coriolis forces for characteristic flow scales within the core ${ }^{13,14}$. Most bodies with active dynamos in the solar system today are estimated to have $R o_{l}$ below a critical value of $\sim 0.12$. Furthermore all bodies other than the ice giants have dipole-dominated instantaneous fields with dipole tilts $<10^{\circ}$, consistent with Coriolis forces strongly influencing their geometries ${ }^{14,15}$. For the Earth's time-averaged field, this is manifested by behavior described by Equation (1).

Synchronous rotation of the Moon was likely established early in lunar history, such that by 3.7 Ga ago the rotation rate was sufficiently slow $\left(\sim 5 \times 10^{-6} \mathrm{rad} \mathrm{s}^{-1}\right)$ that its $R o_{l}$ would have reached a value of $\sim 2$, suggesting that the field in the core was dominantly nondipolar ${ }^{14}$. In particular, simulations of dynamos with $R o_{l}$ exceeding a critical value of $\sim 0.1$ have found that such dynamos have a dipolarity, 
$f_{d i p}=\frac{B_{d}}{B_{n d}}=0.001-0.1$, where $B_{d}$ and $B_{n d}$ are the intensities of the dipole (e.g., spherical harmonic degree $l=1$ ) and non-dipole (e.g., $l>1$ ) components of the field, respectively. On the other hand, the small size of the lunar core $\left(\sim \frac{1}{7}\right.$ of the body's radius $\left.{ }^{16}\right)$ should lead to significant attenuation of the higher order multipolar terms at the lunar surface. Conservatively assuming that at the top of the lunar core the multipolar field was dominated by the quadrupolar term, such that $B_{d}=0.001-0.1$ and the quadrupolar part of the field was $B_{q}=0.9-0.999$, then given that dipole (e.g., degree $l=1$ ) and quadrupole terms $(l=2)$ fall off as $r^{-(l+2)}$ for distance from the top of the core, $r$, the predicted field at the surface would still be significantly multipolar $\left(f_{\text {dip }} \sim 0.007-0.44\right)$. Therefore, we do not expect that the lunar dynamo must have followed Equation (1).

Even so, a high $R o_{l}$ does not by itself preclude a dipolar surface magnetic field geometry. The multipolar state implied by the high $R o_{l}$ only applies to the field-generating region in the core, whereas paleomagnetic observations only constrain the surface field. For example, as has been proposed for Mercury which has a dominantly dipolar field in spite of its high estimated $R o_{l}{ }^{17,18,19}$, a subadiabatic, stably stratified layer may have existed at the top of the lunar core (e.g. ref. 16) that suppressed multipolar field components due to their higher time variability relative to that of the low-degree field. In addition, if the core is stratified, then the thermal and compositional buoyancy forces must be considered separately to account for double-diffusive convection. However, previous estimates of the lunar $R o_{l}$ were calculated using a co-density approach, which may only be applicable to turbulent, well-mixed cores $^{14}$. It has been demonstrated that using a double-diffusion approach instead can drastically change the predicted surface magnetic fields ${ }^{20}$. Additionally, previous estimates of the lunar $R o_{l}$ assumed that dynamo action is convection-driven ${ }^{14}$ but this assumption may not apply for a different mechanism of dynamo generation, such as precession ${ }^{21,22}$.

Although there is strong evidence for the existence of a past lunar dynamo, its physical mechanism and power source are highly uncertain. The small size of the lunar core means that the apparent surface field paleointensities before $3.5 \mathrm{Ga}$ ago imply a very strong core dynamo field $(>24 \mathrm{mT})$ even in the limiting case of a purely dipolar field. As a result, convective core dynamo scaling laws fail by more than an order of magnitude to generate surface intensities $>50 \mu \mathrm{T}$ over time periods lasting $>30 \mathrm{Ma}^{23}$. Precession-driven dynamos, although potentially able to achieve higher paleointensities ${ }^{21}$, struggle to generate magnetic fields in spherical cores for realistic viscosities ${ }^{24}$ although the dissipative heat from precession might instead serve as a heat source for a convective dynamo ${ }^{22}$. Impact-driven 
dynamos that initiate mantle stirring of the core may be able to generate the observed paleointensities, but can only be sustained for thousands of years ${ }^{25}$ and require basin-forming impacts which ceased before $3.7 \mathrm{Ga} \mathrm{ago}^{26}$. It has also been proposed that a basal magma ocean could generate the observed intensity and longevity of the lunar dynamo, but this requires the magma ocean to have an exceptionally high electrical conductivity ${ }^{27}$.

Measurements of the geometry of the ancient lunar magnetic field would provide invaluable constraints for distinguishing between these potential dynamo mechanisms (Figure 1). As discussed above, convective core dynamos in fast rotating bodies $\left(R o_{l}<0.12\right)$ are expected to be predominantly dipolar (Figure 1a), whereas the lunar dynamo is expected to be multipolar (Figure 1b). If the magnetic field was generated by a highly conductive silicate basal mantle magma ocean ${ }^{27}$, even higher degrees of multipolarity may be observable given the shallower source region (Figure 1c,d).

The above theoretical challenges with generating a lunar field by the dynamo process has long motivated a diversity of other proposed non-dynamo mechanisms. Although these alternative mechanism are unlikely to explain the bulk of the observed paleomagnetic record and in many cases are inconsistent with other lunar data, highly speculative, and/or not well-developed theoretically, they may conceivably have accounted for some lunar paleomagnetism. As such, some of these non-dynamo models make first-order predictions for the lunar paleofield geometry that could be assessed with paleodirectional measurements under the assumption of no subsequent true polar wander.

If the lunar surface was magnetized by Earth's time-averaged geocentric axial dipole ${ }^{28}$ a uniform inclination equal to the lunar latitude would be observed (Figure 1e). Another possibility is that the lunar surface was magnetized by the interplanetary magnetic field (IMF), which is expected, on average, to have been instantaneously aligned along the plane of the lunar equator (Figure 1f) ${ }^{29}$. Magnetic fields amplified by basin-forming impacts in the solar wind, although recently shown to be unable to explain lunar paleointensities ${ }^{30}$, were predicted to have inclinations close to horizontal $\left(0^{\circ}\right)$ over much of the lunar surface and close to vertical $\left(90^{\circ}\right)$ at the basin antipodes (Figure $\left.1 \mathrm{~g}\right)^{31}$. Magnetic fields generated locally by smaller impacts would likely have scattered inclinations that cluster around $0^{\circ}$ for near-vertical impacts in near-zero ambient fields (Figures $1 \mathrm{~h}$ ) ${ }^{32,33,34,35}$. Near-horizontal inclinations might be expected for a thermoelectric dynamo generated by adjacent lava basins ${ }^{36}$, while near-vertical inclinations might be expected for a unipolar dynamo in an isolated lava basin ${ }^{28}$ (Figure 1i,j). Cometary impacts perhaps generated predominantly horizontal inclinations across the lunar 
surface (Figure 1k) $28,37,38$.

To constrain the geometry and intensity of the ancient lunar dynamo, we studied two mare basalt samples collected during the Apollo 17 mission: 75035 and 75055 . These two samples have indistinguishable ages of $3753 \pm 9 \mathrm{Ma}$ and $3752 \pm 9 \mathrm{Ma}$, respectively ${ }^{39}$. They were collected from within $\sim 10 \mathrm{~m}$ of the rim of the 610 -m-diameter, $\sim 500 \pm 200 \mathrm{Ma}$ old Camelot crater ${ }^{4}$, located at $20.2^{\circ} \mathrm{N}$, $37.3^{\circ} \mathrm{E}^{40}$. The Camelot crater event is thought to have exposed $>120 \mathrm{~m}$ of mare basalt flow stratigraphy. The significance of 75035 and 75055 for paleomagnetic studies only recently came to light following reassessment of the geology of the Taurus-Littrow valley ${ }^{4}$. Although they were originally interpreted as ejecta blocks, the parent boulders of these samples are now thought to represent near in-situ wall rock exposed during mass wasting of Camelot Crater. It is unlikely that these samples have moved more than $\sim 10 \mathrm{~m}$ from their original location, although they may have been differentially tilted or rotated with respect to the underlying bedrock ${ }^{4}$. A similar mass wasting process was observed at the Apollo 15 site Hadley Rille whose eastern rim is composed of a mixture of fractured bed rock and associated locally-derived boulders ${ }^{41}$.

The parent boulder for 75055, as well as surrounding blocks, exhibit clear layering and planar features that can be used to infer paleohorizontality (see Methods 1.1 and Figure S3). 75055 is therefore one of very few known Apollo samples whose original orientation can be unambiguously constrained. We have used this sample to measure the paleoinclination (along with the paleointensity) of the lunar field 3.7 Ga ago. Sample 75035 was also acquired from a parent boulder with possible layering features, but the small number of astronaut photographs and their limited range of perspectives means that we cannot unambiguously reconstruct paleohorizontal for this sample. We therefore report only paleointensity data for 75035 .

Electron microscopy data indicate samples 75035 and 75055 contain essentially pure-Fe kamacite $(\alpha-\mathrm{Fe})$ with a dominantly multidomain grain size (Supplementary Text S1 and S2). However, isothermal remanent magnetization (IRM) acquisition data indicate 75035 and 75055 contain grains with coercivites $>1000 \mathrm{mT}$ and $>400 \mathrm{mT}$, respectively, indicating the presence of a population of pseudosingle domain (e.g., single vortex) grains (Supplementary Text S1 and S2). Both 75035 and 75055 have cooling rates $<3{ }^{\circ} \mathrm{C} \mathrm{hr}^{-1}$ constrained by their petrographic textures ${ }^{42,43}$, and therefore cooled too slowly to have recorded putative plasma fields or impact fields, which would have had a maximum duration of $<1$ day. Additionally, neither sample exhibits evidence for shock metamorphism 
(see Supplementary Text S3.3), indicating they never experienced a pressure wave with an amplitude greater than $\sim 5 \mathrm{GPa}$ (the Hugoniot elastic limit for lunar rocks ${ }^{44,45}$ ), suggesting they are unlikely to have acquired a shock remanent magnetization. The samples should therefore primarily contain a thermoremanent magnetization (TRM) acquired during primary cooling on the Moon after their emplacement.

Our alternating field (AF) demagnetization of specimens of 75035 (taken $>2.5 \mathrm{~mm}$ from a JSC bandsawn surface) and 75055 (which has never been cut by JSC bandsaw) revealed a non-origintrending low coercivity (LC) component of natural remanent magnetization (NRM) that was removed by $0-11 \mathrm{mT}$ (Figure 2 and Table S10). The LC components are highly scattered within each parent sample (Figure 3). Once the LC overprints were removed, we found that six non-bandsawn specimens of 75035 and five specimens of 75055 each contained a final origin-trending high coercivity (HC) component that was blocked up to $40-60 \mathrm{mT}$ and essentially unidirectional within each parent sample (Figure 2 and Table S10). The Fisher mean anisotropy-corrected HC direction for these six specimens of 75035 is $030^{\circ} / 11^{\circ}$ in present-day lunar coordinates (see Methods Table 1 for definitions and descriptions of coordinate systems) with a $95 \%$ confidence interval $\alpha_{95}=16^{\circ}$. The Fisher mean anisotropy-corrected $\mathrm{HC}$ direction for five specimens of 75055 in present-day lunar coordinates is $334^{\circ} / 02^{\circ}$ with $\alpha_{95}=11^{\circ}$. In lunar paleohorizontal coordinates, the $\mathrm{HC}$ component of 75055 has a paleoinclination of $I_{L P H}=34^{\circ}$, $\alpha_{95}=11^{\circ}$, and an estimated Fisher precision parameter $\kappa=46.0$, where the uncertainty is estimated from the scatter in the $\mathrm{HC}$ directions among individual specimens.

We measured mean $\mathrm{HC}$ paleointensities of $50.7 \pm 13.5 \mu \mathrm{T}$ and $57.0 \pm 17.3 \mu \mathrm{T}(1 \sigma$ standard error of 4 and 8 subsamples) for 75035 and 75055 , respectively, using the anhysteretic remanent magnetization (ARM) method (Figure S19 and Table S9). The calculated confidence intervals for individual specimens reported in Table S9 are based on $95 \%$ confidence intervals from the least-squares fits of NRM lost versus ARM lost during AF demagnetization and do not take into account systematic uncertainties due to the poorly known ratio of ARM to TRM. The latter $2 \sigma$ uncertainties are estimated to be a factor of $\sim 5^{1}$, such the true range of paleointensities is $10-250 \mu \mathrm{T}$ and $11-285 \mu \mathrm{T}$ for 75035 and 75055 , respectively. Therefore, the paleointensity estimates for 75035 and 75055 are both within error of the mean value of $77 \mu \mathrm{T}$ measured from six samples of age $3.5-4.2 \mathrm{Ga}^{5,26,46,47}$. They thereby provide additional evidence that $>3.5 \mathrm{Ga}$ ago, the Moon had an active dynamo generating a strong magnetic field. Even the lower limit on our recovered paleointensity $(\sim 10 \mu \mathrm{T})$ exceeds the field strength predicted 
by convective dynamo scaling laws and is barely compatible with a dynamo intermittently reaching this strength over an integrated time of just $30 \mathrm{Ma}^{23}$. Furthermore, this minimum paleointensity is still a factor of 2 stronger than the weak paleointensities measured between $\sim 3.5-1$ Ga ago, and at least an order of magnitude stronger than the weakest fields that can be reasonably attributed to an active dynamo $2,3,48$.

We consider the implications of our paleoinclination results based on two alternative assumptions about 75055's paleoinclination record; first, that it reflects only the instantaneous lunar magnetic field and, second, that it is representative of the time-averaged (over $\sim 0.1-10 \mathrm{Ma}$ ) lunar field (i.e., that secular variation on these timescales is negligible). Considering the instantaneous magnetic field, we calculated the location of the virtual magnetic pole under the assumption of a selenocentric axial dipole. Since declination and the sign of the inclination are unconstrained, the possible virtual magnetic pole locations define two small circles (Figure 4c). For our recovered paleoinclination, the implied dipole tilt includes $0-10^{\circ}$, consistent with an axially-aligned instantaneous magnetic field that is dipole-dominated like those of other inner solar system bodies ${ }^{15,49,50}$.

We next consider our paleoinclination results assuming the instantaneous and time-averaged lunar magnetic fields were similar. In this case, the recorded inclination would reflect a durable feature of the lunar field geometry rather than a localized, transient state like those associated with core waves or short-lived convection rolls. First, we use our paleoinclination results to assess the different magnetic field sources that could have magnetized the lunar surface (further details are provided in Supplementary Text S3). Second, we consider a multipolar core dynamo field, and, finally, we consider the implications for true polar wander. To assess different magnetic field sources, we assume that Camelot crater was at its present latitude at the time of NRM acquisition. Using Equation 1 and assuming no subsequent true polar wander, the magnetic inclination of a selenocentric axial dipole at Camelot crater would be $\sim 36^{\circ}$, which is indistinguishable from our paleoinclination estimates (Figure $4 \mathrm{a}$ ). External field sources from either Earth's ancient dynamo or the IMF are expected to produce inclinations that are too low or high at the latitude of Camelot crater, respectively, under the assumption that their orientations were similar to those observed today (Figure 4a). Other sources of magnetization such as impacts and thermoelectric dynamos (Figure 1g-k) are also apparently inconsistent with our paleoinclination results.

Although our recovered paleoinclination is consistent with a selenocentric axial dipole generated 
by a core dynamo, we show next that the results do not require such a geometry. In particular, we consider non-axial dipolar and multipolar fields (see Supplementary Text S3.1 for details). As a limiting case, we investigated how the addition of zonal quadrupolar and octupolar components to an axial dipole will effect the predicted magnetic field inclination at the latitude of Camelot crater (Figure 4b). Even just considering these low order, purely zonal terms, our paleoinclination cannot exclude a high degree of multipolarity. A multipolar magnetic field is consistent with the low lunar rotation rate, and would also be favored by dynamo sources closer to the surface (e.g., a basal magma ocean ${ }^{27}$ ) given the $r^{-(l+2)}$ dependence of multipolar fields. Clustering of paleopoles near the poles and the equator has been observed by numerous crustal magnetic studies ${ }^{10,51}$ and would be consistent with a predominantly quadrupolar dynamo or else a persistent non-axial dipole field ${ }^{52}$.

Finally, we consider the implications of our paleoinclination for true polar wander, assuming that our measured paleoinclination corresponds to a selenocentric axial dipole. The topography and gravity of the Moon suggest that the rotation axis may have reoriented by $36 \pm 4^{\circ}$ from an ancient paleopole location of $54 \pm 5^{\circ} \mathrm{N}, 309 \pm 6^{\circ} \mathrm{E}$ since the Cassini state transition ${ }^{46}$. True polar wander has also been suggested to explain variability in the directions of crustal magnetization ${ }^{10,53}$. Lunar hydrogen deposits, which may represent ancient polar ice deposits, are observed in two distinct, antipodal locations offset from the current spin poles, suggesting that the Moon may have experienced a significant degree of true polar wander ${ }^{7}$. Assuming a selenocentric axial dipole that may have reversed, our paleoinclination measurement predicts paleopole locations offset from the spin axis that are indistinguishable from several independent studies at $\sim 3.7 \mathrm{Ga}$ ago ${ }^{5,6,7,52}$ (Figure 4c). However, no subsequent true polar wander is also indistinguishable from our measured paleoinclination.

The novelty of this study is that we were able to reconstruct the paleohorizontal orientation of the parent block for 75055 from planar features documented in astronaut photographs. This has enabled an accurate measurement of the absolute paleoinclination of a lunar sample relative to a known latitude on the lunar surface. The recovered paleoinclination of $34 \pm 11^{\circ}$ at a latitude of $20.2^{\circ} \mathrm{N}$ is consistent with, but does not require, a selenocentric axial dipole. In addition, our paleointensity measurements have shown that both 75035 and 75055 cooled in the presence of a strong field ( $>40 \mu \mathrm{T}$ ), consistent with previous evidence for an epoch of intense magnetic fields $\sim 3.5-4.2 \mathrm{Ga}$ ago $^{1}$.

Our data do not exclude the possibility of a significantly multipolar lunar magnetic field (Figure 4b). Nevertheless, under the assumption of a selenocentric axial dipole, we find that allowed $3.7 \mathrm{Ga}$ true 
polar wander paths are consistent with those of independent crustal magnetic and hydrogen deposit studies $^{5,6,7}$ (Figure 4c). A dipolar magnetic field is at odds with the high $R o_{l}$ of the Moon, which could indicate stratification of the lunar core ${ }^{16,18,19}$ or a lunar dynamo operating in a non-convective regime, such as precession ${ }^{21,22}$.

We have provided a framework in which other potentially orientable Apollo samples can be included to improve our understanding of how the lunar magnetic field varied with latitude and its implications for the lunar dynamo. Co-temporal measurements of the ancient lunar field from samples at different latitudes on the Moon will enable the magnetic field geometry to be unambiguously determined. Paleoinclination measurements from samples of different ages would also allow the rate and extent of true polar wander to be better defined, as well as allowing lunar secular variation to be quantified. Future sample return missions to the Moon should collect oriented samples from confirmed bedrock from a range of latitudes and formation ages, which will greatly enhance our understanding of the geometry and temporal variability of the ancient lunar magnetic field and the mechanism of dynamo generation. 


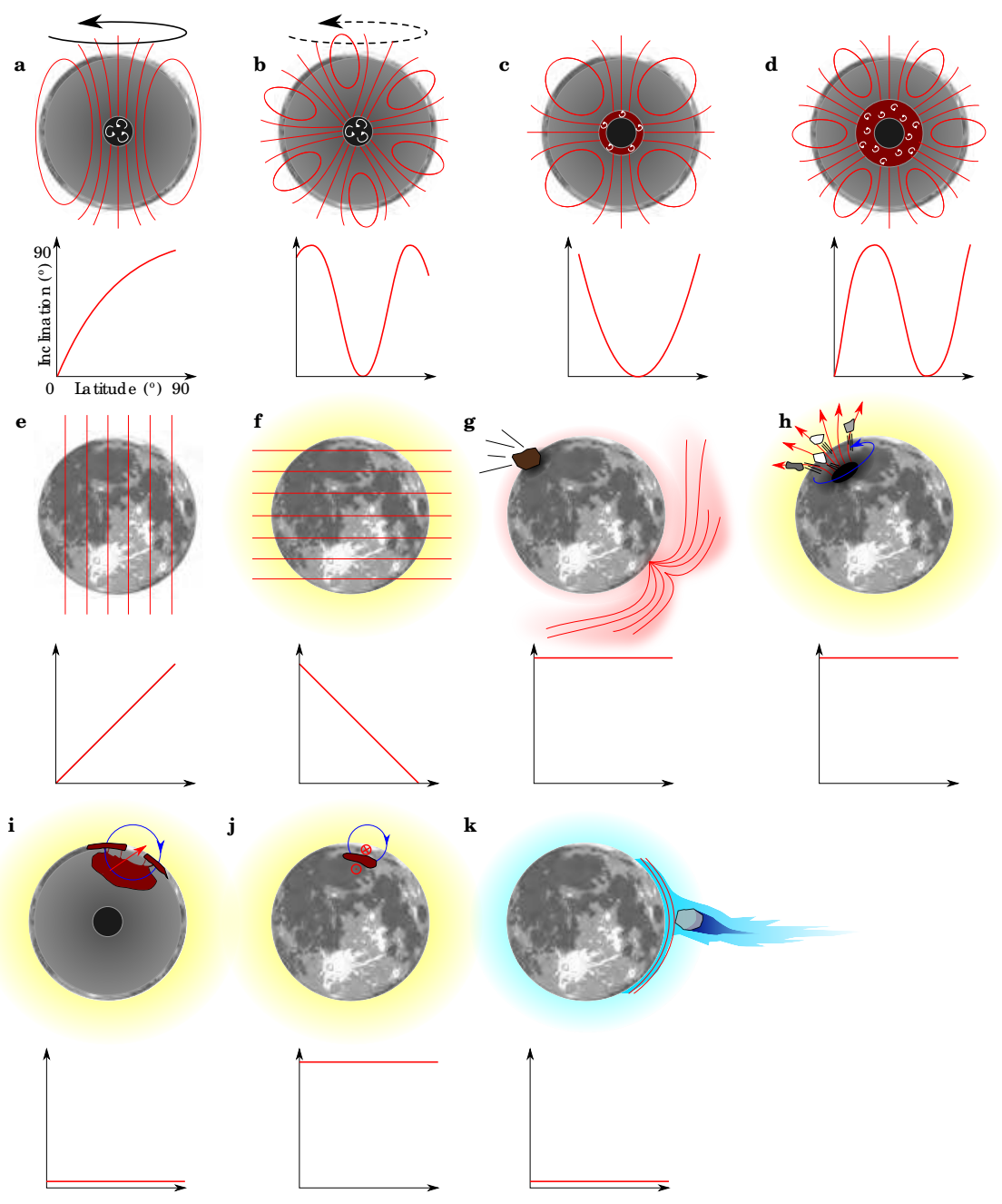

Figure 1 | Predictions of various hypothesized lunar magnetic field sources for the paleoinclination of the lunar field. The top and bottom of each panel show a schematic of the approximate field direction with respect to the Moon and the inclination as a function of latitude, respectively. The black region represents the lunar core and dark red regions represent molten silicate. In all cases, red lines depict the magnetic field, blue lines depict any currents generated, and the yellow region around the Moon represents the solar wind when this is specifically required to generate the proposed magnetic field. a, A selenocentric axial dipolar magnetic field generated by a core dynamo with a rapid planetary rotation rate $\left(R o_{l}<0.12\right)$. b, A multipolar, non-axisymmetric magnetic field generated by a core dynamo with a slow planetary rotation rate $\left(R o_{l}>0.12\right)$. c, An axially-aligned quadrupolar dynamo generated by a deep-sourced magma ocean dynamo ${ }^{27}$. d, An axially-aligned octupolar dynamo generated by a shallow-sourced magma ocean dynamo ${ }^{27}$. $\mathbf{e}$, The Earth's mean axial dipole field at the location of the Moon. $\mathbf{f}$, The IMF. g, Antipodal field amplified by basin forming impacts ${ }^{54}$. $\mathbf{h}$, Fields generated by impact plasmas ${ }^{28}$. The predicted inclination is for rocks directly below the crater. i, Magnetic fields generated by the thermoelectric effect in lava basins that are electrically connected to subsurface magma and the solar wind ${ }^{28,36}$. j, Magnetic fields generated by a unipolar dynamo in lava basins ${ }^{28}$. The predicted inclination is for a rock at the surface close to the lava pool. $\mathbf{k}$, Compression of the IMF around the Moon by a cometary coma ${ }^{28}$. 


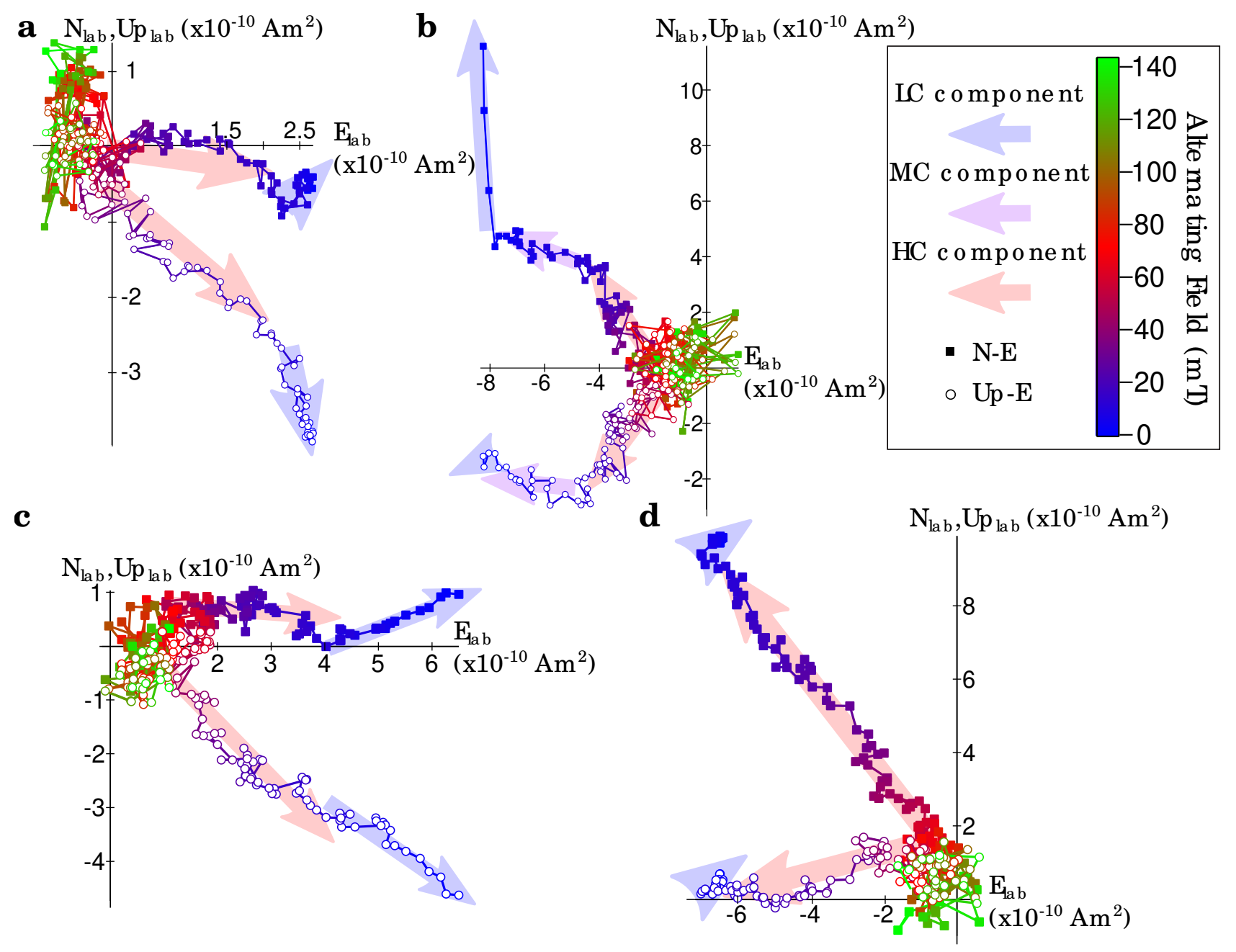

Figure 2 Demagnetization of NRM in 75035 and 75055. Orthographic projections in laboratory coordinates show the NRM vector during AF demagnetization projected along the North-East (closed squares) and Up-East (open symbols) directions. Stable components are shown by blue (LC), purple (medium coercivity, $\mathrm{MC}$ ) and red $(\mathrm{HC})$ arrows, respectively. Data shown here are not corrected for remanence anisotropy. The peak AF is shown by the colour bar. a, Specimen 75035-242Ae. b, Specimen 75055-127Aa. c, Specimen 75035-242Bg. d, Specimen 75055$127 \mathrm{Ae}$. 

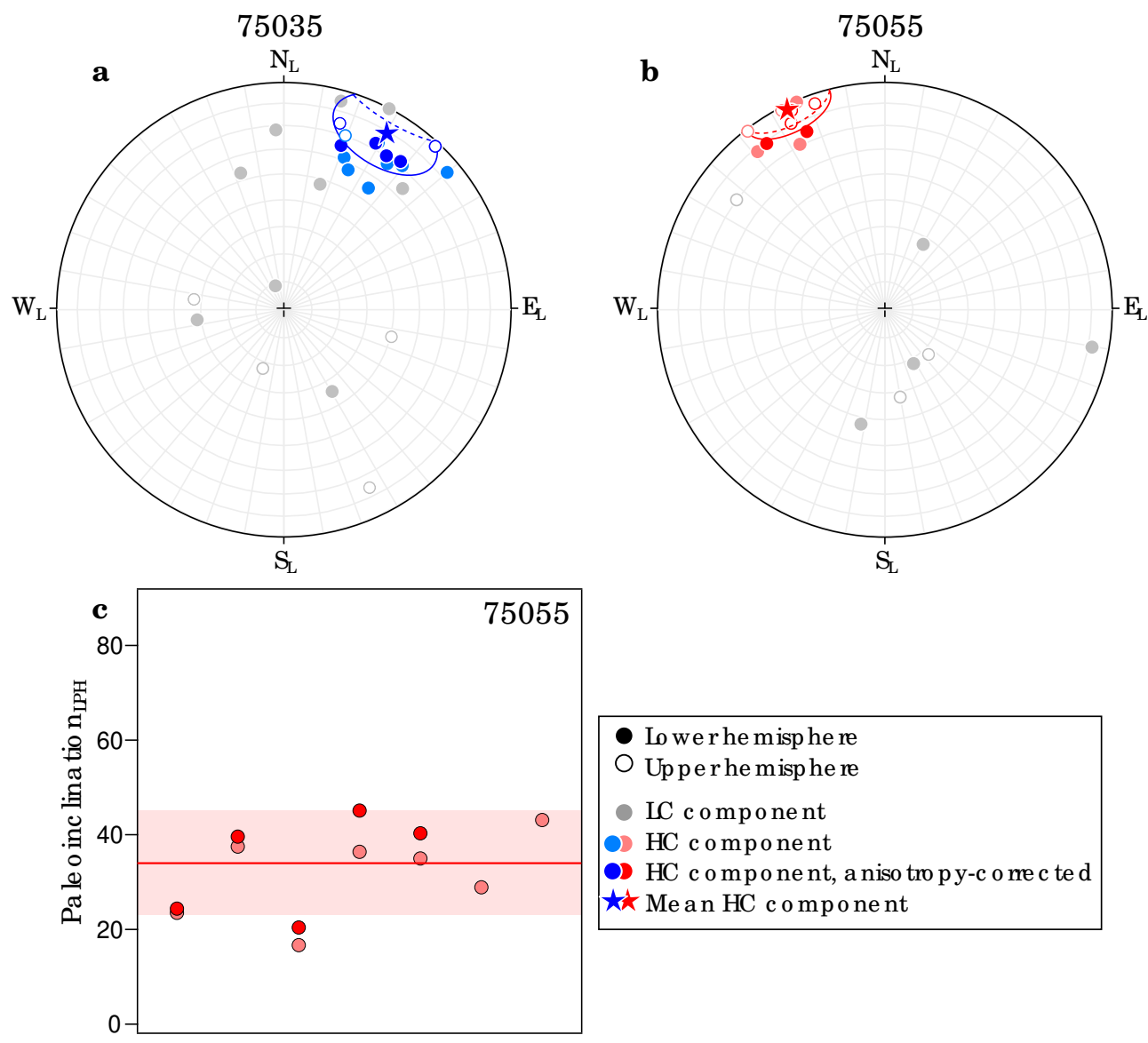

Lower he misphere

O Upperhemisphere

- LC c omponent

- $\mathrm{HC}$ c omponent

2) HC c omponent, a niso tro py-c o rec te d

$\star \star$ Mean HC component

Figure 3 | NRM components in 75035 and 75055 and associated paleoinclinations. The LC (grey) and $\mathrm{HC}$ (pale blue for 75035, pale red for 75055) components are shown in present-day lunar coordinates for individual specimens. Remanence-anisotropy-corrected HC components are shown in bright blue and red for 75035 and 75055 , respectively. The mean (stars) and $95 \%$ confidence interval (ellipses) were calculated for the anisotropy-corrected HC components. a, Equal area stereonet projections of mutually oriented specimen components for 75035 in lunar coordinates. b. Equal area stereonet projections of mutually oriented specimen components for 75055 in lunar coordinates. c, Paleoinclinations in lunar paleohorizontal coordinates for the HC components in 75055. The mean (horizontal red line) and one standard deviation (red shaded region) are shown for anisotropy-corrected components. 

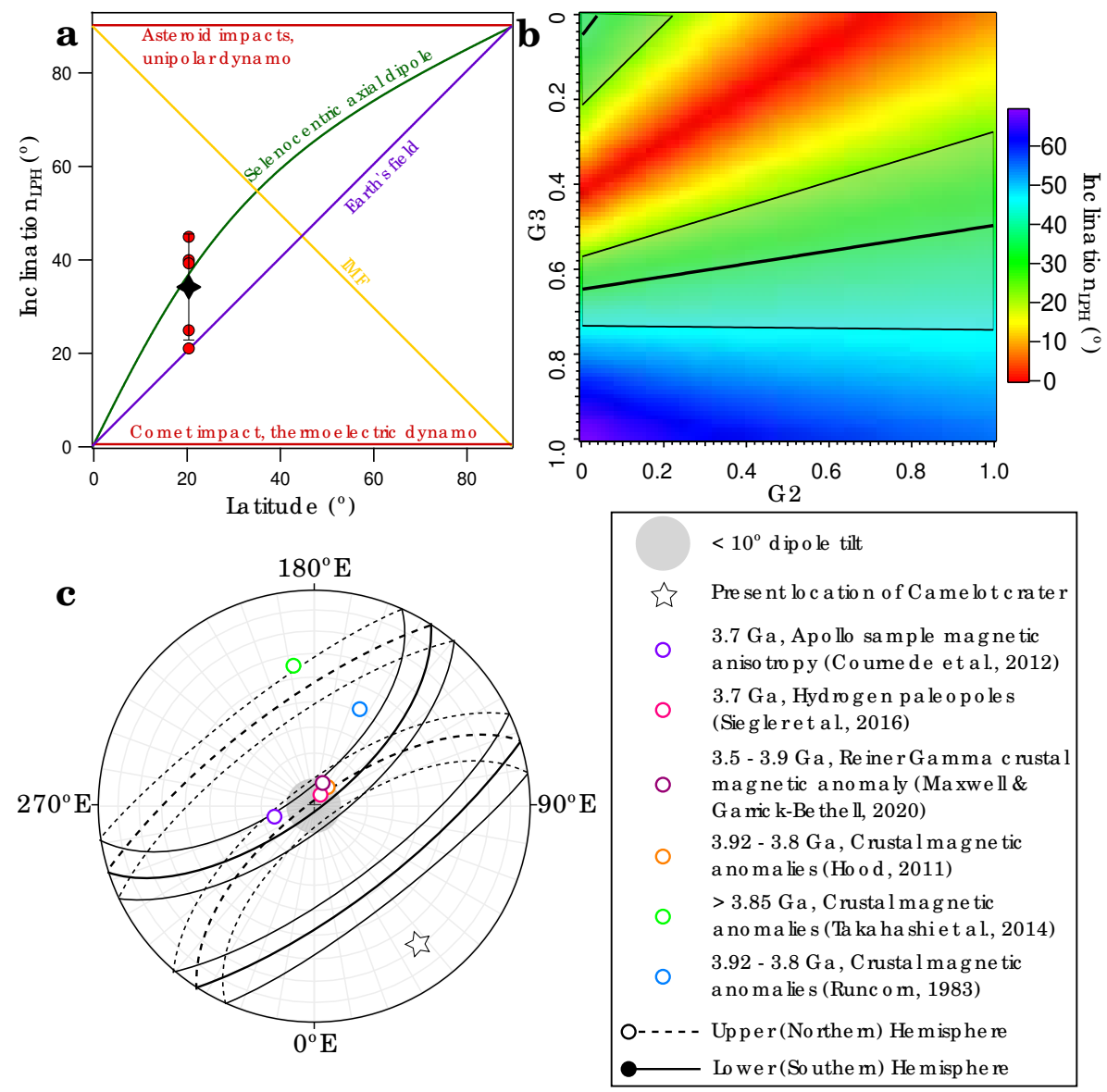

Figure 4 Predictions for the paleoinclination of the lunar field, and implications for multipolarity and true polar wander. a, Paleoinclination results are shown at the present latitude of Camelot crater by the black symbol with $95 \%$ confidence error bars. Red dots are the measured paleoinclinations from each specimen of 75055. Predicted inclination versus latitude curves (see Figure 1) are shown for impact fields, a unipolar dynamo and a thermoelectric dynamo (red lines), Earth's magnetic field (purple line), the IMF (yellow line), and a selenocentric axial dipole (green line). b. The predicted magnetic inclination at Camelot crater for a magnetic field with zonal dipolar, quadrupolar and octupolar components, where $G 2$ and $G 3$ are the quadrupolar to dipolar and octupolar to dipolar field ratios, respectively. The thick black line is the mean paleoinclination and the regions bounded by thin black lines represent the 95\% confidence interval. Both low and high degrees of multipolarity are permitted by our measured paleoinclination. c, Equal area stereographic projection showing the possible paleopole locations $3.7 \mathrm{Ga}$ ago in present-day lunar geographic coordinates. Open symbols/dashed lines and closed symbols/solid lines are in the northern and southern hemisphere, respectively. The star is the current location of Camelot crater where the samples were collected. The thick black lines are the permitted locations of the north pole calculated from our mean recovered paleoinclination. Thin black lines are the 95\% confidence interval on our measurement. The grey shaded region represents $<10^{\circ}$ dipole tilt. North 3.7 Ga paleopole locations from independent studies are shown in purple ${ }^{5}$, pink ${ }^{7}$, red ${ }^{52}$, orange $^{6}$, green $^{51}$ and blue ${ }^{55}$. 


\section{Methods}

\subsection{Reconstructing sample orientation relative to the lunar surface}

In this study, we analyzed subsamples 75035,242 and 75055,127, which B.P. Weiss and H.H. Schmitt selected and oriented at JSC in 2016. Our subsamples are oriented with respect to their parent samples in JSC coordinates (see Table 1 for details of coordinate systems). We reconstructed the orientations of parent samples of 75035 and 75055 relative to their sampling positions on the lunar surface using JSC and astronaut photographs and transcripts recorded by astronaut H.H. Schmitt and astronaut E.A. Cernan during sample collection. Both 75035 and 75055 were hammered directly off blocks with visible planar features at the edge of Camelot crater by astronaut H.H. Schmitt in December 1972 (Figure S2). The blocks were photographed before and after sampling by astronauts H.H. Schmitt and E.A. Cernan. The present-day orientations of 75035 and 75055 relative to each block on the lunar surface were reconstructed using photographs taken in lunar-lighting conditions at the Lunar Receiving Laboratory ${ }^{56}$. The astronaut photographs indicate that the blocks were likely not undisturbed bedrock when samples 75035 and 75055 were collected but rather had undergone subsequent tilting and rotation since their formation (Figure S3). As a result, outcrop textural features allow the paleohorizontal to be determined, but cannot account for the paleoazimuthal direction. We therefore only attempt to constrain paleohorizontal and determine the ancient field's paleoinclination and not paleodeclination.

Planar features are observed on the parent boulders of 75035 and 75055 and on other blocks around Camelot crater (Figure S3). The astronauts noted that these planar features were largely defined by variations in vesicle distribution ${ }^{57}$ and may represent the tops of lunar lava flows. A terrestrial analogue to these lava flows are the extensively studied Hawaiian basalts, with the low viscosity lava flows on the lunar surface having an emplacement mechanism comparable to that of pāhoehoe flows ${ }^{42,58,59}$. Pāhoehoe flows grow via inflation due to injection of fresh magma beneath a solid crust. This results in a distinct distribution of vesicles, with the bottom and top of the lava flow containing horizontallyaligned 'vesicular zones' which can be tens of centimetres to metres in thickness, while horizontal 'vesicle sheets' ( $\sim 10 \mathrm{~cm}$ thickness) form near the centre of the flow. 75035 and 75055 are thought to have formed in the central part of a pāhoehoe-like flow given the coarse $(>1 \mathrm{~mm})$ crystalline nature of ilmenite within the samples ${ }^{42,60}$ such that the planar features observed on the parent boulder of 75055 likely correspond to vesicle sheets. The spatial distribution of features is consistent with a lava flow 
thickness of $<10 \mathrm{~m}$. Lunar lava flows have a lower viscosity than that of any known terrestrial flow ${ }^{61}$ and formed thin $(<10 \mathrm{~m})$ and laterally extensive flows ${ }^{62}$. It should also be noted that horizontal vesicular layers can be produced without the injection of fresh melt into the system ${ }^{63}$. In terrestrial basalts exhibiting vesicular layers, such as the Columbia River flood basalt flows, vesicles have been observed to form approximately every $1 \mathrm{~m}$, consistent with theoretical calculations ${ }^{63}$. However, given the lower viscosity of lunar basalts and lunar gravity, these layers are likely to be closer together on the Moon due to faster rise times and are therefore a plausible explanation for the $\sim 30 \mathrm{~cm}$ spacing between vesicle layers observed on the blocks at Camelot crater (Figure S3). We therefore assumed that the planar features defined by vesicles within 75055's parent block represent the paleohorizontal at the time of eruption. Using this assumption, we reconstructed the paleohorizontal orientation of the block. Note also that the similar compositions of the blocks at the crater edge and their spatial distribution indicate that they are close to their formation localities such that the measured paleointensities can be interpreted in the context of the latitude of Camelot crater.

We used photographs of sample 75055 (in JSC coordinates) to reconstruct its original orientation in lunar geographic coordinates (Figure S6). The attitude of the visible foliations on the parent boulder of 75055 were reconstructed by measuring the apparent trend and plunge of planar features observable in a series of photographs taken from different locations (Table S2 and Figure S7). A great circle was fitted to the trend and plunge measurements to define the plane. The planar features have a strike and dip of $243^{\circ} / 36^{\circ}\left(\alpha_{95}=5^{\circ}\right)$ in lunar coordinates. The orientation of the plane was used to tilt the boulder back to its original paleohorizontal position. Once the paleohorizontal orientation of the parent boulder of 75055 was established, the magnetic paleoinclination was recovered. Because the lunar magnetic field may have undergone reversals, it is only meaningful to recover the magnitude of the paleoinclination and not its sign. 


\begin{tabular}{lcl}
\hline Coordinate System & Abbreviation & Description \\
\hline Magnetometer & mag & $\begin{array}{l}\text { The coordinate system of the superconducting rock magnetometer for AARM measurements at the Fort } \\
\text { Hoofddijk Paleomagnetic Laboratory. }\end{array}$ \\
Laboratory & lab & Orientation used in the MIT Paleomagnetism Laboratory. \\
JSC & JSC & Orientation used at the JSC to document Apollo samples. \\
Present-day lunar & L & Orientation on the lunar surface, where Top is outward normal from the lunar surface and North is horizontal \\
& and toward the present-day spin axis. & 75035,75055 \\
Lunar paleohorizontal & Using planar features on blocks, lunar coordinates are tilt corrected to the paleohorizontal. Only inclination & 75035 \\
& is considered in this coordinate system.
\end{tabular}

Table 1 | Definitions and descriptions of the coordinate systems used throughout this study. The order in which coordinate systems are listed is the order in which corrections were made to sample orientations throughout the study.

\section{$1.2 \quad$ Paleomagnetic analysis}

All demagnetization experiments were conducted on a $2 \mathrm{G}$ Enterprises Superconducting Rock Magnetometer 755 in the MIT Paleomagnetism Laboratory. The magnetometer has a sensitivity of $<1 \times 10^{-12} \mathrm{Am}^{2}$ (ref. 64). 15 specimens of 75035 and 9 specimens of 75055 were demagnetized using AF because thermal demagnetization may cause sample alteration (Figure S12) ${ }^{65,66}$. The NRM was removed by three axis $\mathrm{AF}$ demagnetization in steps of $0.5 \mathrm{mT}$ up to $25 \mathrm{mT}$, steps of $1 \mathrm{mT}$ up to $95 \mathrm{mT}$, and then steps of $1.5 \mathrm{mT}$ up to $145 \mathrm{mT}$. The magnetic moment was measured after each $\mathrm{AF}$ step and the three orthogonal measurements were then averaged to correct for gyroscopic remanent magnetization (GRM) following the Zijderveld-Dunlop method ${ }^{65,67}$. A subset of specimens (75055127Aa, 75055-127Ab, 75055-127Ac, 75055-127Ae and 75055-127b) were demagnetized up to $420 \mathrm{mT}$ in steps of $7.5 \mathrm{mT}$ to ensure $\mathrm{HC}$ components were entirely removed.

NRM components were characterized using principal component analysis. Origin-trending components were identified when the maximum angular deviation (MAD) exceeds angular deviation (DANG). The final fits for such origin-trending components were anchored to the origin ${ }^{68,69}$. These fits were used to calculate a Fisher mean direction and associated 95\% confidence interval $\left(\alpha_{95}\right)$ for each specimen in $\mathrm{PMagPy}^{70}$. Ten specimens of 75035 exhibited resolvable origin-trending $\mathrm{HC}$ components, but two of these were disregarded (75035Ad and 75035Ah) since they were partially overprinted by bandsawing at JSC (Figures S1 and S4). Five specimens of 75055 exhibited resolvable origin-trending HC components. Component directions were plotted in Stereonet ${ }^{71,72}$, rotated to present-day lunar coordinates and, for 75055, tilt-corrected using the paleohorizontal estimates from Section 1.1. Recovered 
component directions were also corrected for anisotropy of ARM (AARM) (see Section 1.3).

Paleointensity estimates were calculated using the ARM paleointensity method ${ }^{48,67,73}$. NRM demagnetization curves were compared to AF demagnetization of an ARM with a $100 \mu \mathrm{T}$ DC bias field applied with a $260 \mathrm{mT}$ AF. The TRM-equivalent paleointensity recorded by the samples is given by

$$
P_{A R M}=\frac{\Delta N R M}{\Delta A R M} \frac{b}{f^{\prime}}
$$

where $b$ is the DC bias field and $f^{\prime}=1.34$ is the TRM/ARM calibration factor experimentally determined using lunar basalts ${ }^{74}$. There are two main uncertainties associated with these paleointensity estimates. First, the least squares slope obtained from $\frac{\triangle N R M}{\triangle A R M}$ has an uncertainty associated with scatter in the data around the best-fit line. Second, the calibration factor $f^{\prime}$ is estimated to have a $2 \sigma$ uncertainty of a factor of $5^{1}$. The uncertainty in $f^{\prime}$ originates from its dependence on ferromagnetic mineralogy including grain size, grain morphology and grain distribution. Cooling-rate corrections are not applied because the heating time ( $\sim 1$ hour $)$ for ARM/TRM calibration experiments is similar to the several-day cooling timescales of 75035 and $75055^{75,76}$.

\subsection{Rock magnetic analysis}

We used several rock magnetic techniques to assess the fidelity and magnetic recording properties of samples 75035 and 75055 . We conducted paleointensity fidelity tests, IRM acquisition, Curie balance analysis and quantified the degree of AARM.

To assess the magnetic recording fidelity of samples 75035 and 75055 , we gave the samples an $\mathrm{ARM}$ in a DC bias field ranging from $5-100 \mu \mathrm{T}$ and quantified how accurately we could recover the paleointensity of this field using the ARM paleointensity method. The ARMs were applied at an AF of $260 \mathrm{mT}$ and were AF demagnetized following the same protocol that was used to demagnetize the NRM. The demagnetization curves were compared to the demagnetization curve of the $100 \mu \mathrm{T}$ ARM. For each applied ARM, the difference, $D^{\prime}=\frac{L-I}{L}$, between the recovered paleointensity $(I)$ and the predicted equivalent TRM $(L)$ and the error, $E=\frac{W}{L}$ based on the $95 \%$ confidence on the retrieved paleointensity $(W)$ were calculated ${ }^{65,77}$. Acceptance criteria for a reliable paleomagnetic recorder are defined by $-50 \%<D^{\prime}<100 \%$ and $E<100 \%$.

We conducted IRM acquisition to assess the coercivity spectrum of magnetic carriers in each sample. 
Specimens were given a saturation IRM of $400 \mathrm{mT}$ and then AF demagnetized. The derivative of the demagnetization curve, $\delta \mathrm{IRM} / \delta \mathrm{B}$, was calculated to infer the coercivity distribution.

The magnetic mineralogy and susceptibility to thermal alteration were assessed using a Curie balance at the Fort Hoofddijk Paleomagnetic Laboratory. A small (3.52 mg) fragment of specimen $75055 \mathrm{Ac}$ was exposed to fields of $100-300 \mathrm{mT}$ during a heating cycle from room temperature to $800{ }^{\circ} \mathrm{C}$ and then back to room temperature in air. The magnetization was measured every second over the course of the experiment, which took $\sim 12$ hours. Raw data were smoothed using a Savitzky-Golay filter ${ }^{78}$

The AARM of seven specimens of 75035 and six specimens of 75055 was measured at the Fort Hoofddijk Paleomagnetic Laboratory using a 2G Enterprises Superconducting Rock Magnetometer. Specimens were given an ARM of $50 \mu \mathrm{T}$ in an AF of $150 \mathrm{mT}$. Samples were given an ARM in 9 distinct orientations: $E_{m a g}, N_{m a g}, T_{m a g}, N E_{m a g}, N W_{m a g}, T E_{m a g}, T W_{m a g}, T N_{m a g}$ and $T S_{m a g}$, with each specimen mounted in a different orientation in laboratory coordinates to remove any directional measurement bias (Table S5). After measuring the magnitude and orientation of the acquired ARM, specimens were AF demagnetized up to $150 \mathrm{mT}$ in three orthogonal directions. The order of ARM and demagnetization steps is shown in Table S6. Specimens were mounted in custom-made glass cubes with three-dimensional-printed sample holders (holder moment $<7 \times 10^{-11} \mathrm{Am}^{2}$ ) to maintain exact orientations throughout. This enabled the estimation of the three principal axes defining the anisotropy ellipsoid, where $p_{1}$ is the maximum, $p_{2}$ is the intermediate and $p_{3}$ is the minimum principal axis of anisotropy $^{79}$. After defining the axes, we calculated the degree of foliation $F=\frac{p_{2}}{p_{3}}$, lineation $L=\frac{p_{1}}{p_{2}}$ and anisotropy $P=\frac{p_{1}}{p_{3}}$ for individual specimens ${ }^{80}$. Anisotropy corrections improved the clustering of directions for 75035 , with the Fisher precision parameter, $\kappa$, increasing from 11.9 to 19.1, while for $75055 \kappa$ decreased slightly from 49.2 to 42.9 (Figure 3 ).

\section{Acknowledgements}

The NASA Solar System Workings Program (grant NNX15AL62G) and the NASA Solar System Exploration Virtual Institute (grant \#NNA14AB01A) for support. C.I.O.N. acknowledges funding support from the Simons Foundation. We thank CAPTEM for the loan of samples. We thank Lennart de Groot, Bertwyn de Groot and the Utrecht University glass workshop for assistance with building a 
customized sample-handling system for anisotropy measurements. We thank Sabine Stanley for helpful

405 discussions on dynamo theory.

\section{${ }_{406}$ Author Contributions}

407 C.I.O.N. and B.P.W wrote the paper. B.P.W. and H.H.S. conceived the study. C.I.O.N., B.L.G., 408 A.B. and J.S. collected the paleomagnetic data. C.I.O.N., B.P.W. and B.L.G. analysed the data and 409 reconstructed paleogeographic sample orientations. A.S.P.R. conducted the impact simulations. 


\section{${ }_{410}$ Supplementary Material}

411

\section{S1 Samples and Methods}

\section{S1.1 Sample characterisation and preparation}

Samples 75035 and 75055 are coarse-grained subophitic, high-Ti ilmenite basalts of chemical type A and textural type $1 \mathrm{~B}^{57}$, suggesting they formed as a result of a higher degree of chemical and mineralogical fractionation relative to other Apollo 17 basalts ${ }^{42,60}$. Sample 75035 has an integrated ${ }^{40} \mathrm{Ar} /{ }^{39} \mathrm{Ar}$ age of $3720 \pm 40 \mathrm{Ma}$ and a Rb-Sr age of $3750 \pm 120 \mathrm{Ma}^{81,82}$, while sample 75055 has an integrated ${ }^{40} \mathrm{Ar} /{ }^{39} \mathrm{Ar}$ of $3760 \pm 50 \mathrm{Ma}$ and a $\mathrm{Rb}-\mathrm{Sr}$ age of $3690 \pm 70 \mathrm{Ma}$ (indistinguishable from those of $75035)^{81,83}$. Recently, more precise $\mathrm{Pb}-\mathrm{Pb}$ ages for 75035 and 75055 have yielded still-indistinguishable ages of $3753 \pm 9 \mathrm{Ma}$ and $3752 \pm 9 \mathrm{Ma}$, respectively ${ }^{39}$.

We analysed subsamples 75035,242 and 75055,127, which B.P. Weiss and H.H. Schmitt acquired from the JSC in 2016 (Figures S8 and S9). Sample 75035,242 was chipped from a slab that had previously been prepared using a bandsaw in 1973. Specimens $75035-242 \mathrm{Ad}$ and $75035-242 \mathrm{Ah}$ were immediately adjacent to the bandsaw cut and have been partially overprinted to coercivities $>15 \mathrm{mT}$ (Figure S1). However, all other specimens were taken $>2.5 \mathrm{~mm}$ from the bandsaw cut and the overprint appears to have had no effect, which is consistent with the findings of ref. 3. Progressive AF applications revealed curved demagnetization trends for specimens 75035-242Ad and 75035-242Ah that we characterized be fitting approximate LC, medium coercivity (MC) and HC components. The residual NRM direction progressively moved toward the HC component defined by pristine specimens, suggesting the bandsaw has only partially overprinted these specimens (Table S1 and Figure S1). This partial overprinting is consistent with previous paleomagnetic studies ${ }^{2,3,48}$. Sample 75055,127 was acquired by chipping a slab (,1) which has never been bandsawn. 
$\mathbf{a}$
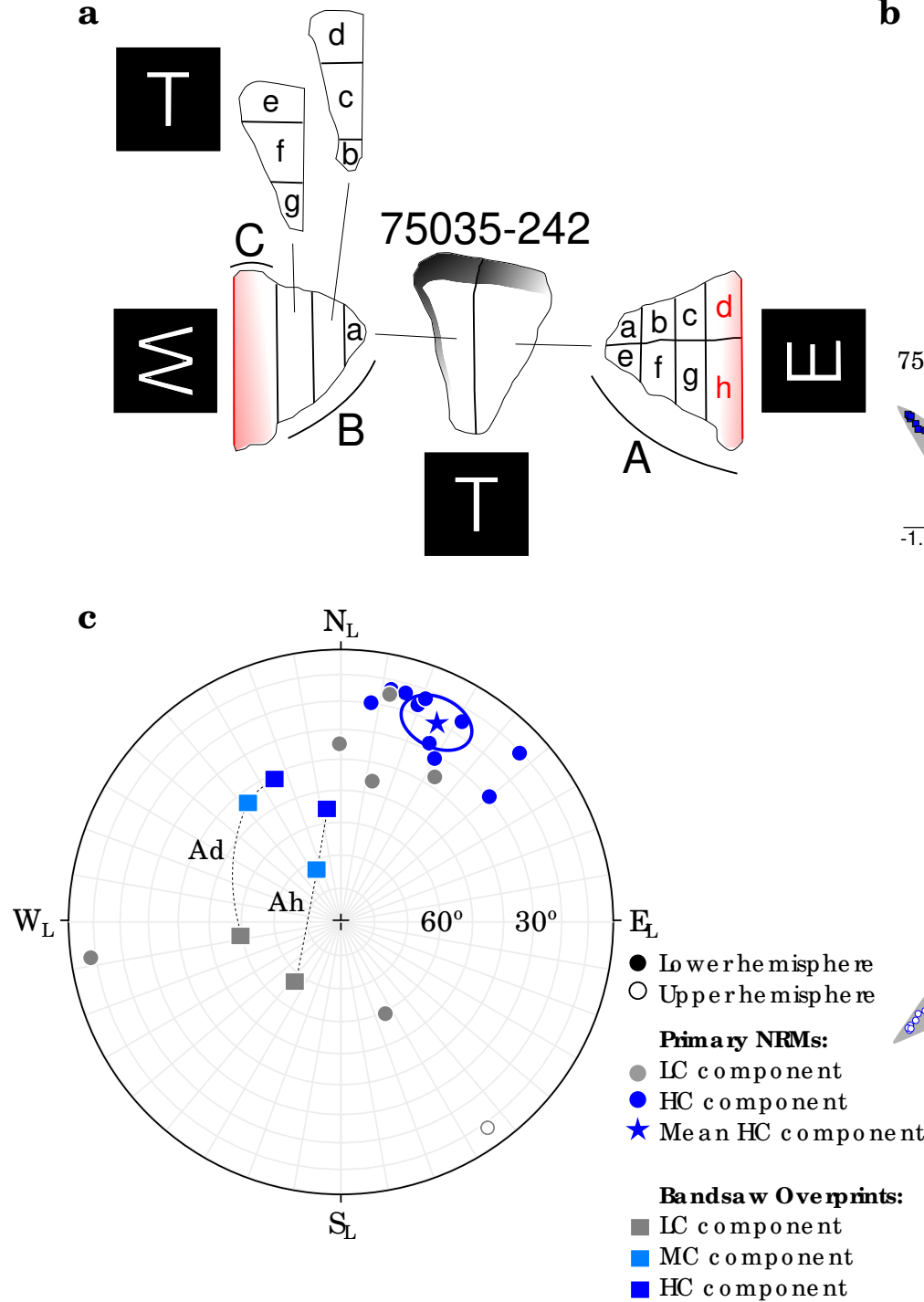

b

75035-242Ad

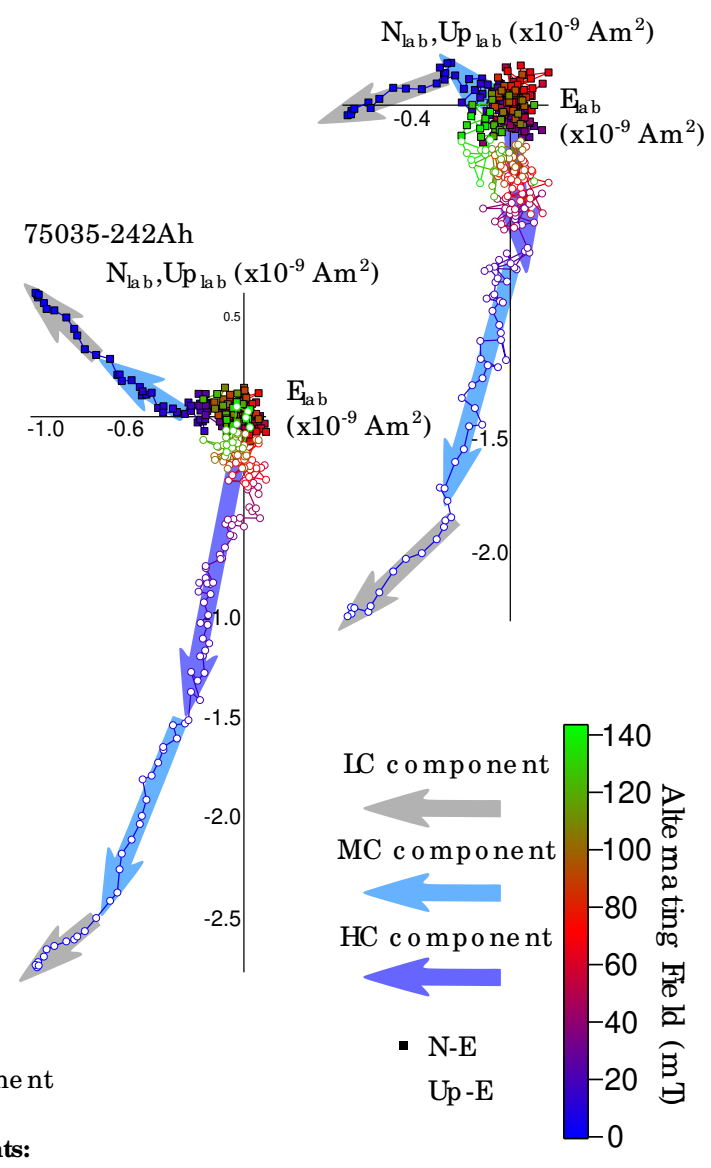

Figure S1 | The influence of bandsaw cutting on the magnetization of specimens of 75035. Orientation cube is $1 \mathrm{~cm}$ across. (a) A diagram showing from where each of our specimens was taken relative to the bandsaw cut. (b) Orthographic projections in laboratory coordinates showing the NRM vector during AF demagnetization for 75035-242Ad and 75035-242Ah in three components projected along the North-East (closed squares) and Up-East (open symbols) directions. Stable components are shown by grey (LC), light blue (medium coercivity, MC) and bright blue (HC) arrows, respectively. (c) Equal area stereographic projection showing the approximate LC, $\mathrm{MC}$ and $\mathrm{HC}$ components for each specimen of 75035 in lunar coordinates. The two specimens that were immediately adjacent to the bandsaw cut (75035-242Ad and 75035-242Ah) were partially overprinted by the bandsaw, as demonstrated by the fact that during AF demagnetization the NRM progressively moves towards the $\mathrm{HC}$ direction defined by the other specimens. All other specimens were acquired $>2.5 \mathrm{~mm}$ from the bandsaw cut and retain their primary magnetization. 


\begin{tabular}{cccccccccc}
\hline Sample & \multicolumn{3}{c}{ LC } & \multicolumn{3}{c}{ MC } & \multicolumn{3}{c}{ HC } \\
& $\mathbf{A F}(\mathbf{m T})$ & $\mathbf{D e c}\left({ }^{\circ}\right)$ & $\operatorname{Inc}\left({ }^{\circ}\right)$ & $\mathbf{A F}(\mathbf{m T})$ & $\mathbf{D e c}\left({ }^{\circ}\right)$ & $\operatorname{Inc}\left({ }^{\circ}\right)$ & $\mathbf{A F}(\mathbf{m T})$ & $\operatorname{Dec}\left({ }^{\circ}\right)$ & $\operatorname{Inc}\left({ }^{\circ}\right)$ \\
\hline $75035-242 \mathrm{Ad}$ & $0-7$ & 245 & 42 & $7-17$ & 298 & 71 & $17-28$ & 324 & 78 \\
$75035-242 \mathrm{Ah}$ & $0-7$ & 222 & 32 & $7-15$ & 213 & 66 & $15-50$ & 185 & 83 \\
\hline
\end{tabular}

Table S1 | LC, MC and HC components of magnetization for bandsaw-overprinted samples 75035-242Ad and 75035-242Ah in laboratory coordinates. The first column lists the sample name, the second, third and fourth columns list the AF range, declination and inclination of the LC component. The fifth, sixth and seventh columns list the AF range, declination and inclination of the MC component. The eight, ninth and tenth columns list the AF range, declination and inclination of the $\mathrm{HC}$ component.

Further subsampling was then conducted in the MIT Paleomagnetism Laboratory using a diamond wire saw in a magnetically shielded clean room (DC field $<200 \mathrm{nT}$ ) to generate specimens for paleomagnetic analysis. Consistent orientations were maintained between all specimens using an orientation cube in laboratory coordinates. For 75035, this orientation system is distinct from JSC coordinates, whereas for 75055, laboratory and JSC coordinates are the same (Table 1). For both samples, the JSC orientation system is distinct from that of present-day lunar geographic coordinates (Table 1). Each specimen was mounted on a 2.5-cm-diameter quartz disc with a magnetic moment $<2 \times 10^{-12} \mathrm{Am}^{2}$ using a small quantity of cyanoacrylate cement. Specimens were mounted in different orientations relative to one another to ensure there is no directional bias induced by the measurement procedure.

In addition to our measurements, previously-measured hysteresis properties of both 75035 and 75055 indicate a dominantly multidomain state ${ }^{84}$. Previous paleomagnetic studies have reported stable (up to a 1 hour unblocking temperature of $\sim 200{ }^{\circ} \mathrm{C}^{85}$ and a peak $\mathrm{AF} \sim 11 \mathrm{mT}^{5}$ ) natural remanent magnetization (NRM) in both samples. An NRM component was identified in $75035^{85}$, but it was unclear whether this was a primary TRM or a secondary overprint ${ }^{86}$. A previous study of 75055 yielded a paleointensity of $\sim 84 \mu \mathrm{T}$ using ARM paleointensity methods ${ }^{5}$. 

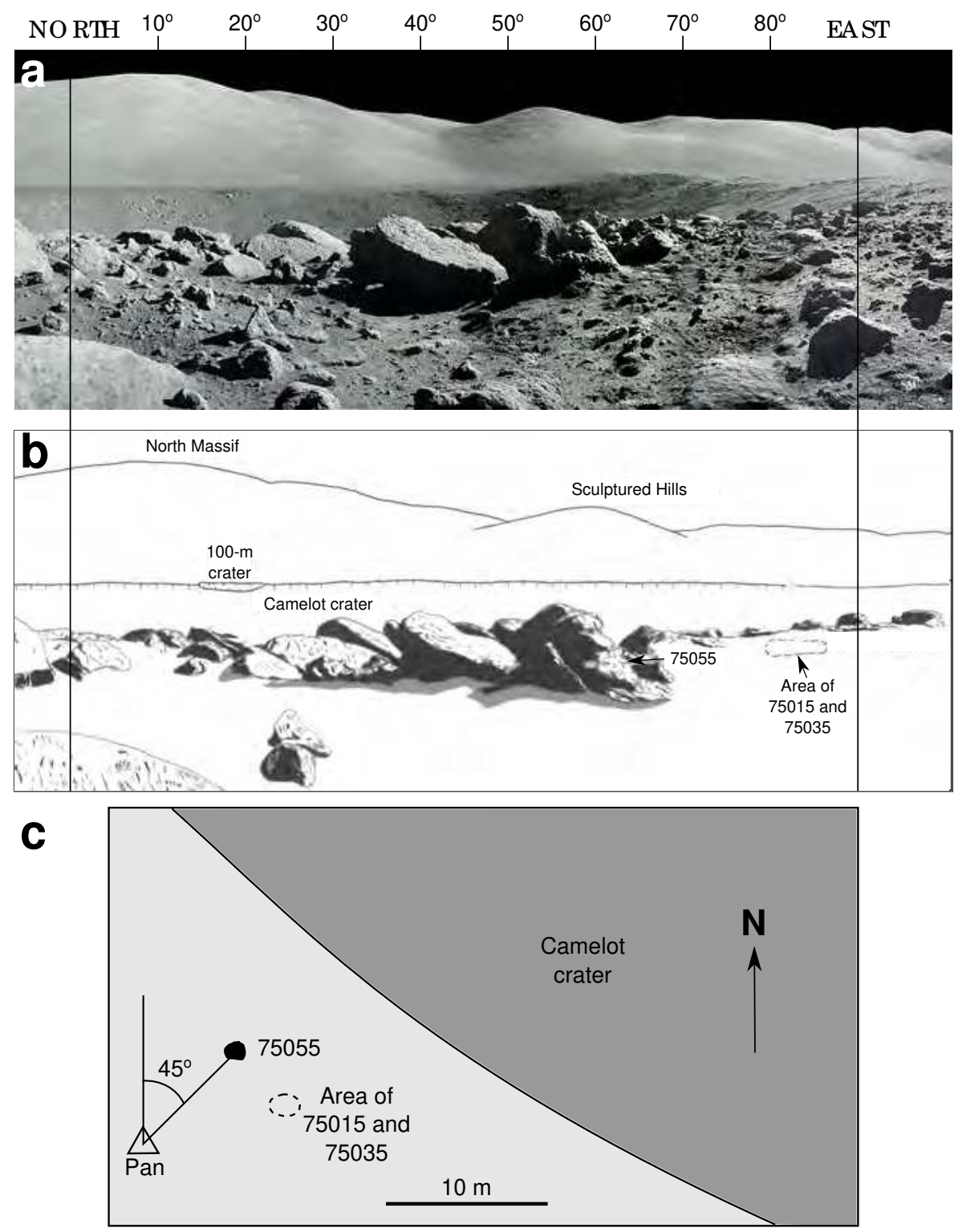

Figure S2 | Geographic context of Station 5 where samples 75035 and 75055 were collected. Figure adapted from ref. 56. (a) Panorama 19 from ref. 56 using astronaut photographs AS17145-22181, 22183, 22159 and 22160. The panorama shows a north-east view of the TaurusLittrow valley. (b) Sketch corresponding to the panoramic photograph in (a). The locations of 75035 and 75055 are shown. (c) Map showing the location from which the panorama was taken and the position of 75035 and 75055 relative to Camelot crater. 


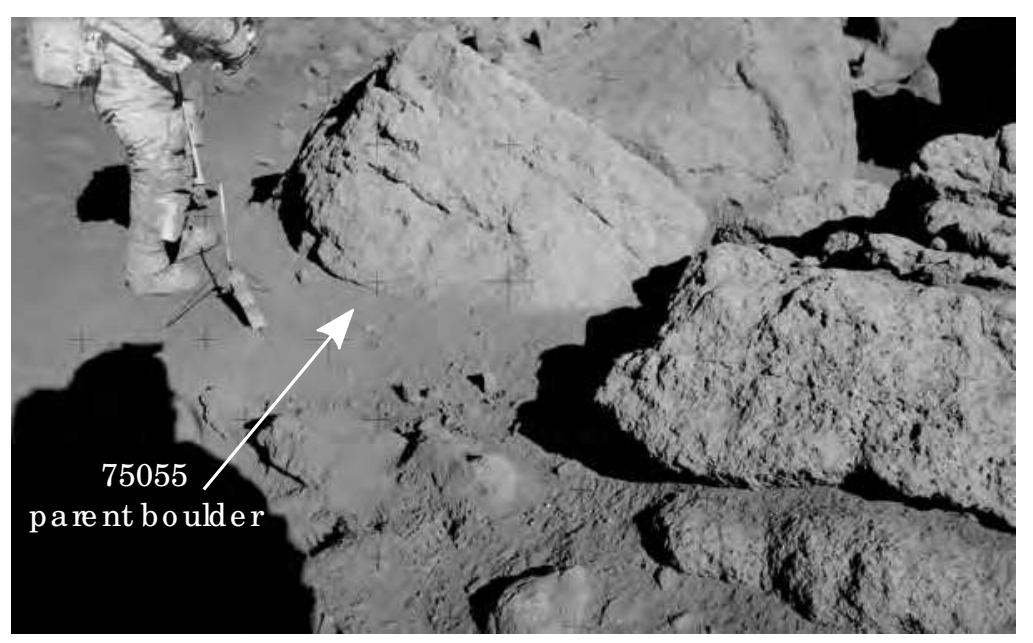

Figure S3 | Panorama using astronaut photographs AS17-133-20330 to AS17-133-20335 showing an example of the planar features in large blocks around Camelot Crater that we used to infer paleohorizontal. Astronaut E.A. Cernan is standing next to the block from which 75055 was sampled.

\section{from laboratory to JSC and JSC to present-day lunar coordinates, respectively:}

In order to translate samples from one coordinate system to another, a series of rotations are made which can also be described by a single $3 \times 3$ matrix. The rotations and associated translation matrices are shown in Figures S4, S5 and S6. The translation matrices $\mathbf{A}_{\mathbf{J S C}}$ and $\mathbf{A}_{\mathbf{L}}$ are used to translate

$$
\left(\begin{array}{c}
N \\
E \\
T
\end{array}\right)_{J S C}=\mathbf{A}_{\mathbf{J S C}} \times\left(\begin{array}{c}
N \\
E \\
T
\end{array}\right)_{l a b}
$$

452

$$
\left(\begin{array}{c}
N \\
E \\
T
\end{array}\right)_{L}=\mathbf{A}_{\mathbf{L}} \times\left(\begin{array}{c}
N \\
E \\
T
\end{array}\right)_{J S C}
$$




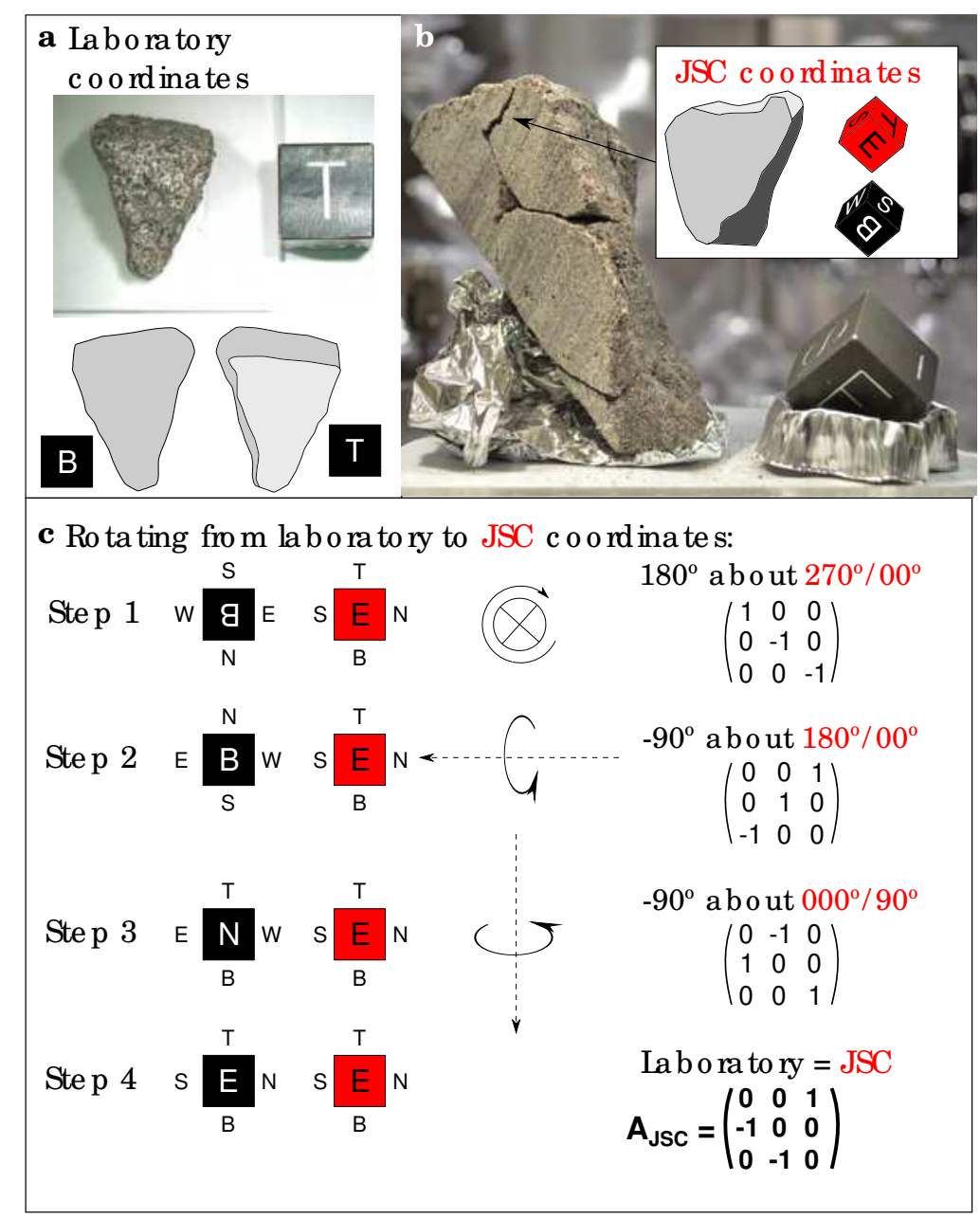

Figure S4 | The four steps to rotate subsample 75035,242 from its orientation in the MIT Paleomagnetism Laboratory to JSC coordinates. Rotations are referenced to the JSC coordinate reference frame. (a) The orientation of the subsample in the MIT Paleomagnetism Laboratory. (b) The orientation of the subsample photographed at the JSC. Note JSC bandsaw surface on the southeast face of the sample. (c) The steps required to rotate from laboratory to JSC coordinates. Laboratory orientation is shown by the black cube and JSC orientation by the red cube throughout. The rotation matrix is shown for each step. The rotation matrix to transform directly from laboratory to JSC coordinates is shown in bold. 

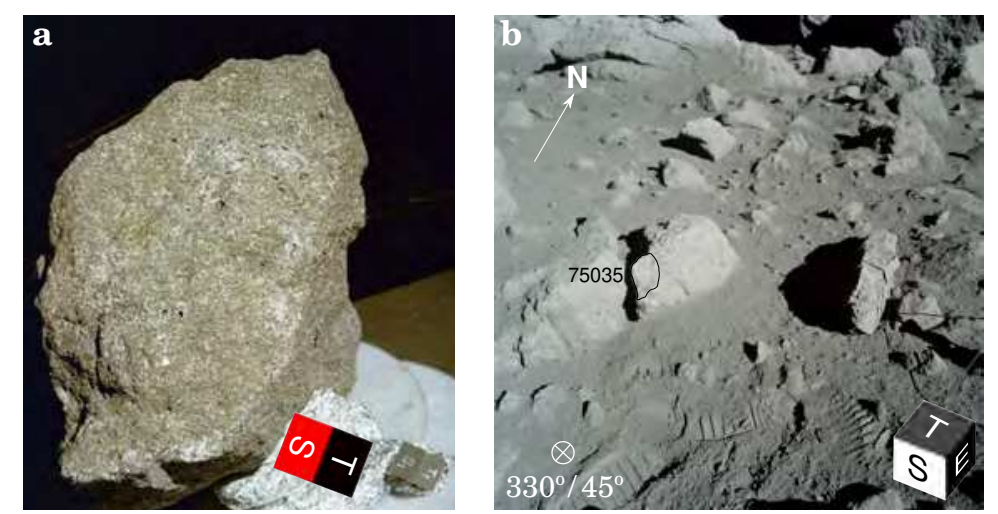

c Rotating from JSC to lunarc o o rdinate s:

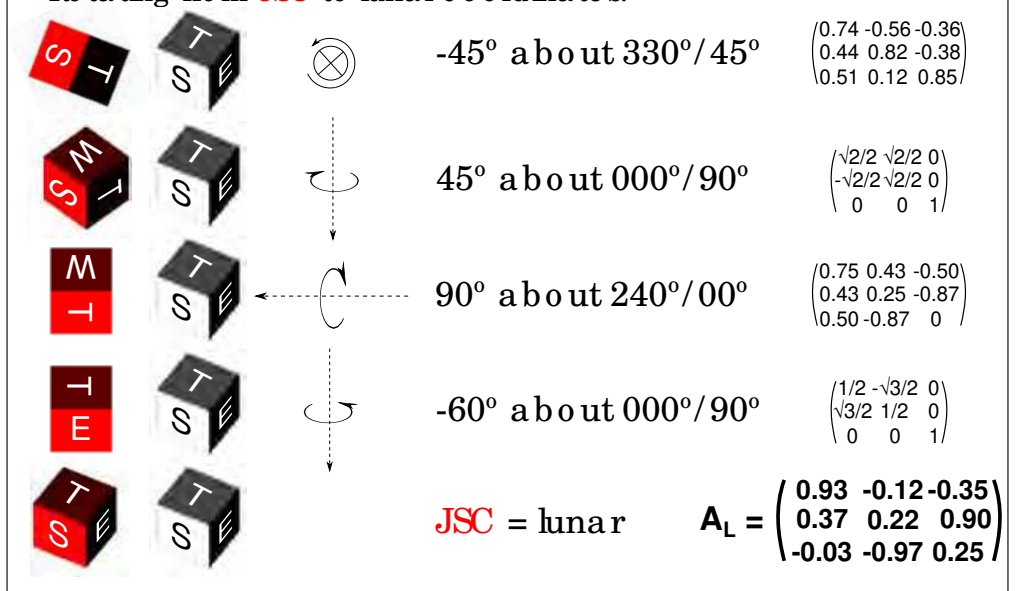

Figure S5 | The steps to rotate sample 75035 from JSC to lunar coordinates. Rotations are made in the lunar coordinate reference frame. (a) JSC photograph S73-19593 showing the orientation of 75035 in JSC coordinates. The sample was illuminated under the same light conditions as those when it was sampled on the Moon. (b) Astronaut photograph AS17-145-22138 showing the orientation of 75035 in lunar coordinates. The direction of North is taken from ref. 56. (c) The steps required to rotate from JSC to lunar coordinates. JSC orientation is shown by the red cube and lunar orientation by the white cube throughout. The rotation matrix is shown for each step. The rotation matrix to transform directly from JSC to lunar coordinates is shown in bold. 

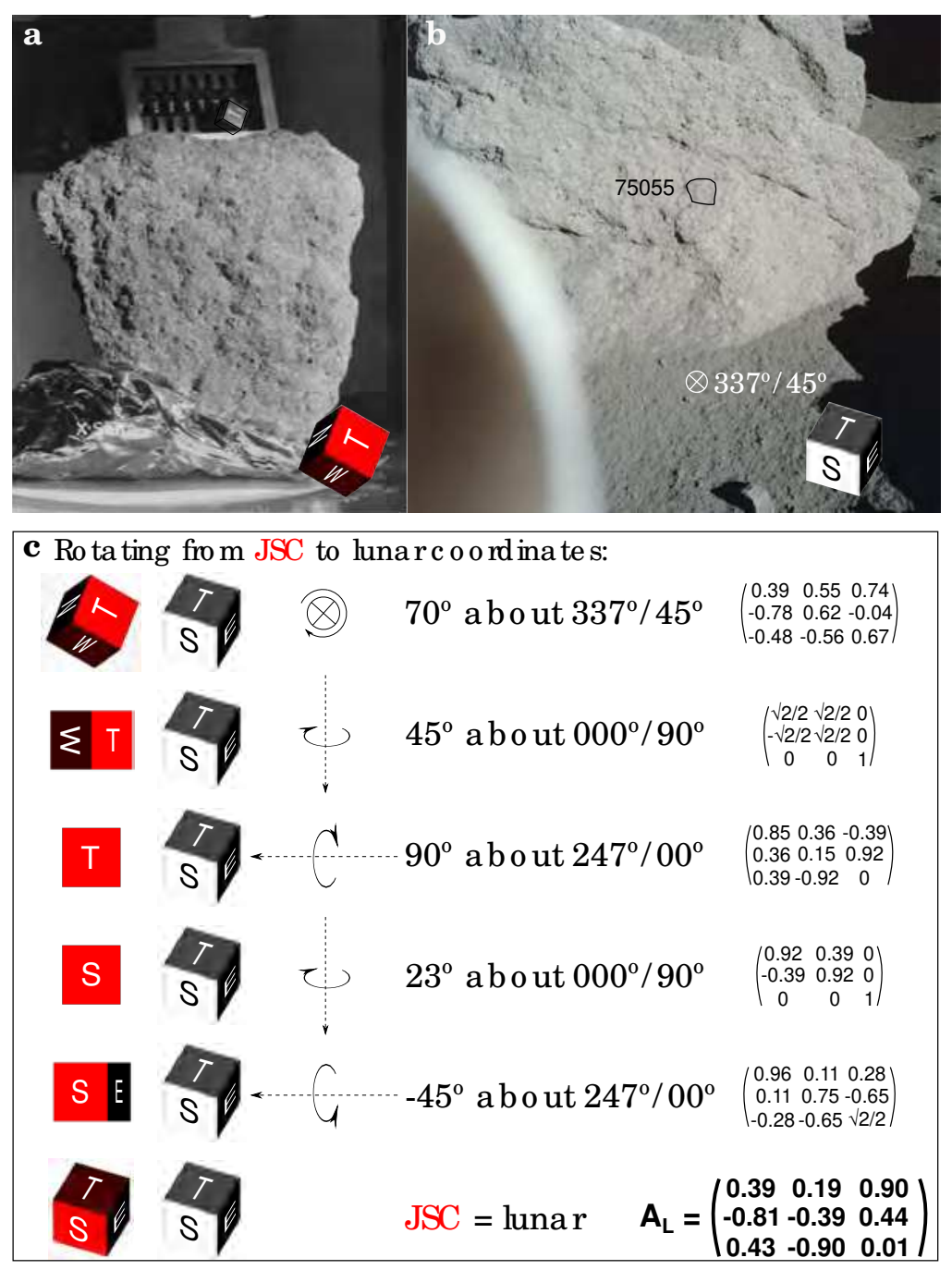

Figure S6 | The steps to rotate sample 75055 from JSC to lunar coordinates. Rotations are made in the lunar coordinate reference frame. (a) JSC photograph S73-17796 showing the orientation of 75055 in JSC coordinates. (b) Astronaut photograph AS17-145-22149 showing the orientation of 75055 in lunar coordinates. The direction of the photograph was determined from the change in trend from Astronaut photograph AS17-145-22141 (see Figures S7 and S2). (c) The steps required to rotate from JSC to lunar coordinates. JSC orientation is shown by the red cube and lunar orientation by the white cube throughout. The rotation matrix is shown for each step. The rotation matrix to transform directly from JSC to lunar coordinates is shown in bold. 

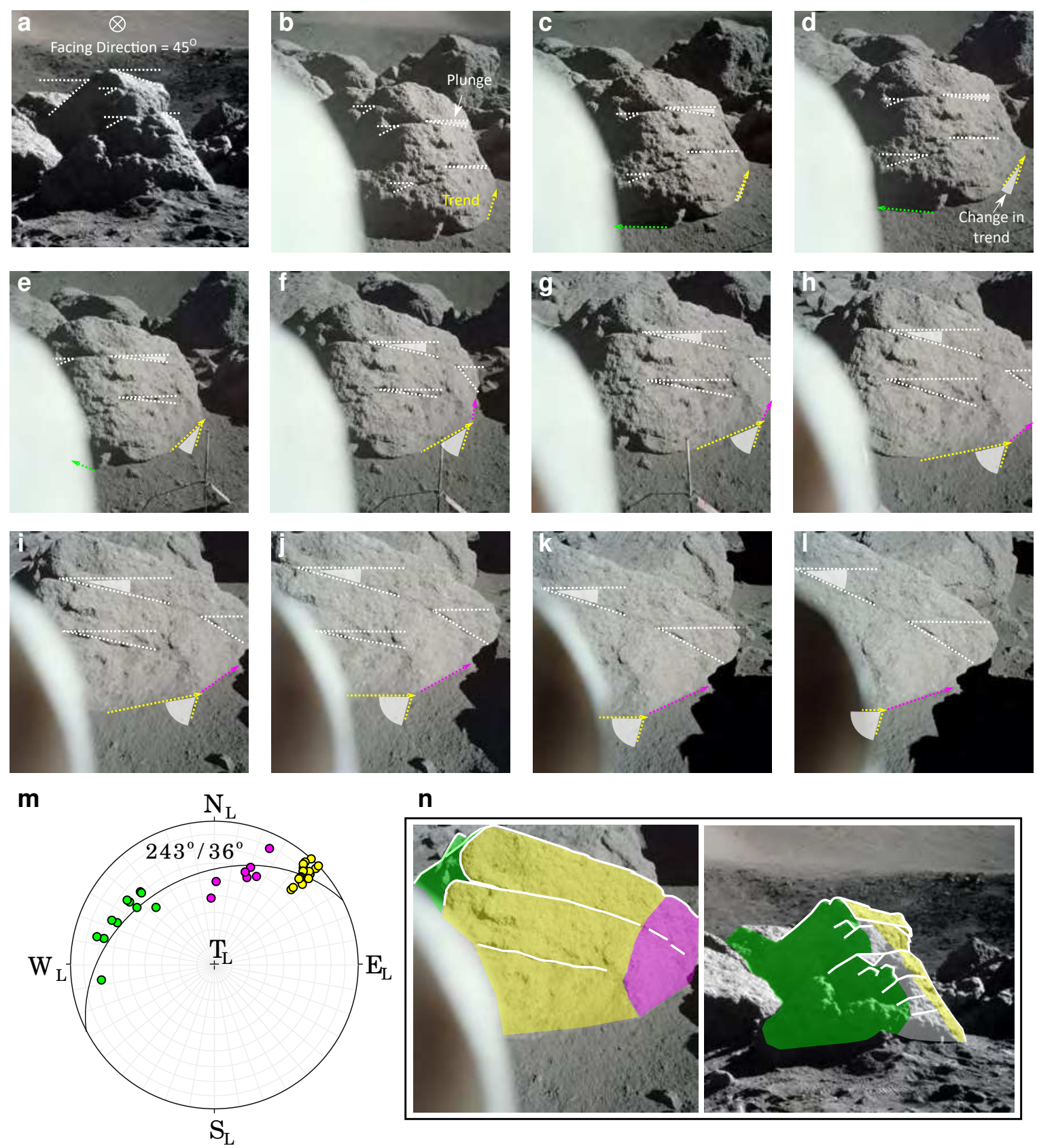

Figure S7 | Estimating the orientation of planar lava flow features on the 75055 parent boulder. Measurements are summarized in Table S2. (a) Astronaut photograph AS17-145-22183. (b) (I) are astronaut photographs AS17-145-22141 to AS17-145-22151 taken from a variety of orientations around the block. The trends and plunges of the planar features were measured as shown by the marked reference features. The colour of the trend arrow corresponds to the face on which the linear features were measured. $(\mathrm{m})$ Equal area stereographic projection showing the orientation of the planar features with a strike and dip of $243^{\circ} / 36^{\circ}$. The plane of best fit was calculated from the measured trends and plunges of linear features identified on three faces of the boulder, coloured green, yellow and pink. (n) Annotated copies of (a) and (g) show the three faces identified on the boulder identified by the green, yellow and pink colours. 


\begin{tabular}{|c|c|c|c|c|c|}
\hline Figure & Photograph & $\begin{array}{c}\text { Direction } \\
\text { of Facing }\left({ }^{\circ}\right)\end{array}$ & $\begin{array}{c}\text { Change } \\
\text { in Trend }\left({ }^{\circ}\right)\end{array}$ & $\begin{array}{l}\text { Plunge } \\
\left({ }^{\circ}\right)\end{array}$ & $\begin{array}{l}\text { Trend } \\
\left({ }^{\circ}\right)\end{array}$ \\
\hline S7a & 22183 & 45 & 0 & 42 & 315 \\
\hline S7a & 22183 & 45 & 0 & 28 & 315 \\
\hline S7a & 22183 & 45 & 0 & 29 & 315 \\
\hline S7a & 22183 & 45 & 0 & 17 & 45 \\
\hline S7a & 22183 & 45 & 0 & 26 & 45 \\
\hline S7b & 22141 & 30 & 15 & 9 & 46 \\
\hline S7b & 22141 & 30 & 15 & 33 & 307 \\
\hline S7b & 22141 & 30 & 15 & 27 & 307 \\
\hline S7b & 22141 & 30 & 15 & 25 & 307 \\
\hline S7b & 22141 & 30 & 15 & 3 & 46 \\
\hline S7c & 22142 & 22 & 23 & 9 & 42 \\
\hline S7c & 22142 & 22 & 23 & 27 & 294 \\
\hline S7c & 22142 & 22 & 23 & 23 & 294 \\
\hline S7c & 22142 & 22 & 23 & 0 & 42 \\
\hline S7d & 22143 & 10 & 43 & 9 & 46 \\
\hline S7d & 22143 & 10 & 43 & 0 & 46 \\
\hline S7d & 22143 & 10 & 43 & 22 & 284 \\
\hline S7d & 22143 & 10 & 43 & 17 & 284 \\
\hline S7e & 22144 & 356 & 49 & 5 & 41 \\
\hline S7e & 22144 & 356 & 49 & 22 & 263 \\
\hline S7e & 22144 & 356 & 49 & 7 & 41 \\
\hline S7f & 22145 & 345 & 60 & 12 & 45 \\
\hline S7f & 22145 & 345 & 60 & 8 & 45 \\
\hline S7f & 22145 & 345 & 60 & 51 & 357 \\
\hline S7g & 22146 & 337 & 68 & 11 & 43 \\
\hline S7g & 22146 & 337 & 68 & 11 & 43 \\
\hline S7g & 22146 & 337 & 68 & 41 & 1 \\
\hline S7h & 22147 & 329 & 76 & 12 & 47 \\
\hline S7h & 22147 & 329 & 76 & 12 & 47 \\
\hline S7h & 22147 & 329 & 76 & 32 & 18 \\
\hline S7i & 22148 & 326 & 79 & 15 & 44 \\
\hline S7i & 22148 & 326 & 79 & 16 & 44 \\
\hline S7i & 22148 & 326 & 79 & 35 & 20 \\
\hline S7j & 22149 & 317 & 88 & 18 & 47 \\
\hline S7j & 22149 & 317 & 88 & 28 & 20 \\
\hline S7k & 22150 & 315 & 90 & 24 & 45 \\
\hline S7k & 22150 & 315 & 90 & 32 & 18 \\
\hline S7I & 22151 & 314 & 91 & 17 & 44 \\
\hline S7I & 22151 & 314 & 91 & 32 & 25 \\
\hline S7I & 22151 & 314 & 91 & 11 & 25 \\
\hline
\end{tabular}

Table S2 $\mid$ Measurements corresponding to Figure S7 for determining the strike and dip of planar features observed on the 75055 parent boulder. The first column lists the part of the figure to which the measurements correspond. The second column lists the astronaut photograph number (each preceded by AS17-254-). The third column lists the facing direction, (i.e., the direction toward which the astronaut took the photograph). The fourth column lists the change in orientation between the photograph and photograph 22183, determined using linear features. The fifth column lists the plunge, which is the angle between horizontal and the linear features from the perspective of each photograph. The sixth column lists the apparent trend of the linear features from the perspective of the facing direction. 

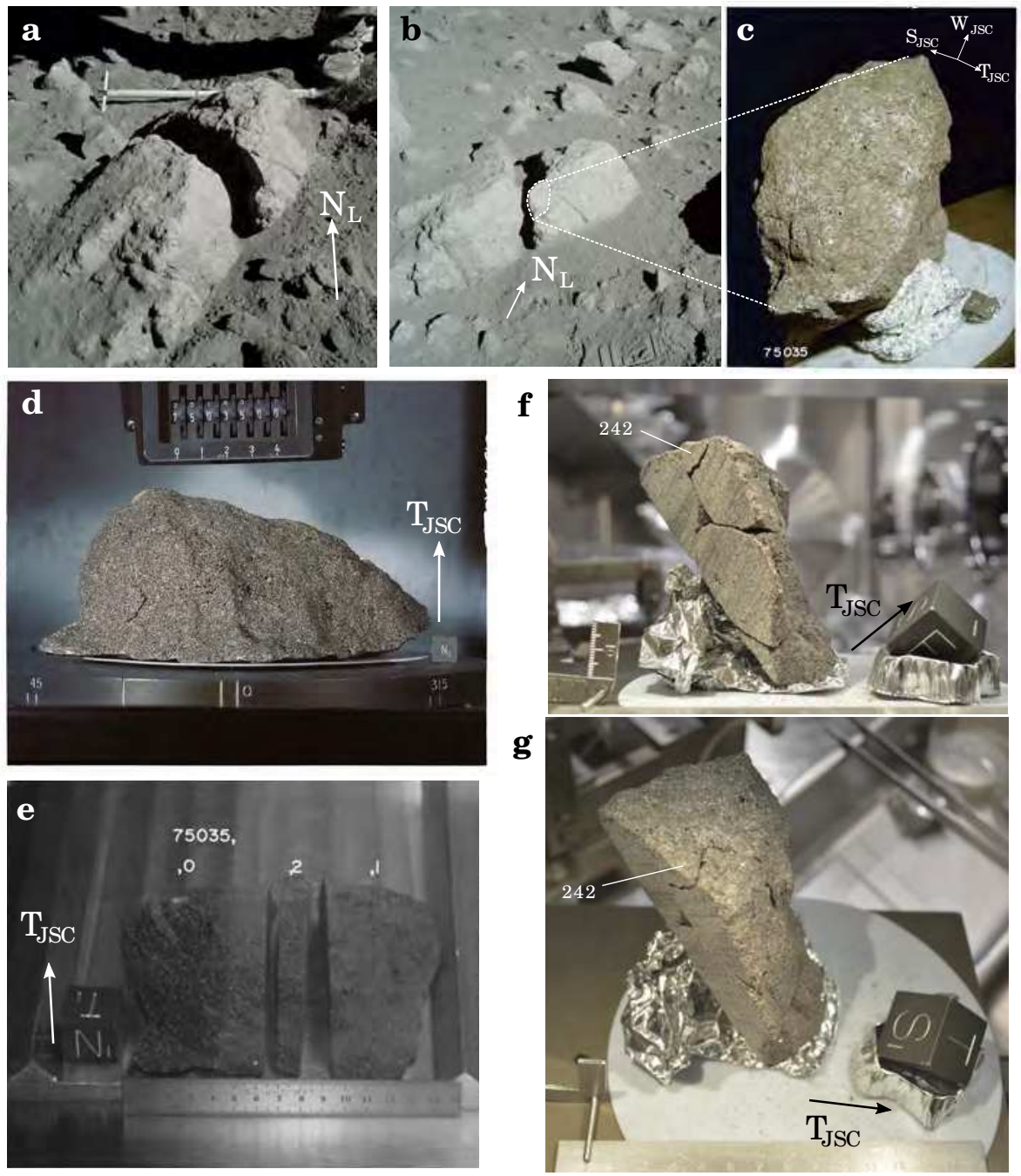

Figure S8 | The location of sample 75035 on the lunar surface and subsequent orientation and sampling at the JSC. (a) Astronaut photograph AS17-145-22139 showing the location of 75035 prior to sampling from the boulder on the lunar surface. $61-\mathrm{cm}$-long hammer for scale. (b) Astronaut photograph AS17-145-22138 showing the side of the boulder and location of 75035 prior to sampling. (c) JSC photograph S73-19593 showing 75035 in JSC coordinates photographed in the same orientation and lighting conditions as when sampled on the Moon. (d) JSC photograph S73-24259 of the north face of 75035 (in JSC coordinates prior to cutting or chipping). The sample cube is $1 \mathrm{~cm}$ across. (e) Photograph S73-31658 showing slabbing of 75035 into three slabs $75035,0,75035,1$ and 75035,2 . Slabbing was carried out using the JSC bandsaw. Subsample 75035,242 is part of 75035,1 . The orientation cube is $2.5 \mathrm{~cm}$ across. (f) Photograph taken during sampling by B.P. Weiss and H.H. Schmitt in 2016 at JSC. Location of sample 75035,242 from cut face (east relative to $2.5 \mathrm{~cm}$ orientation cube). (g) Photograph taken during sampling by B.P. Weiss and H.H. Schmitt in 2016 at JSC. Location of sample 75035,242 from the top (south relative to $2.5 \mathrm{~cm}$ orientation cube). 

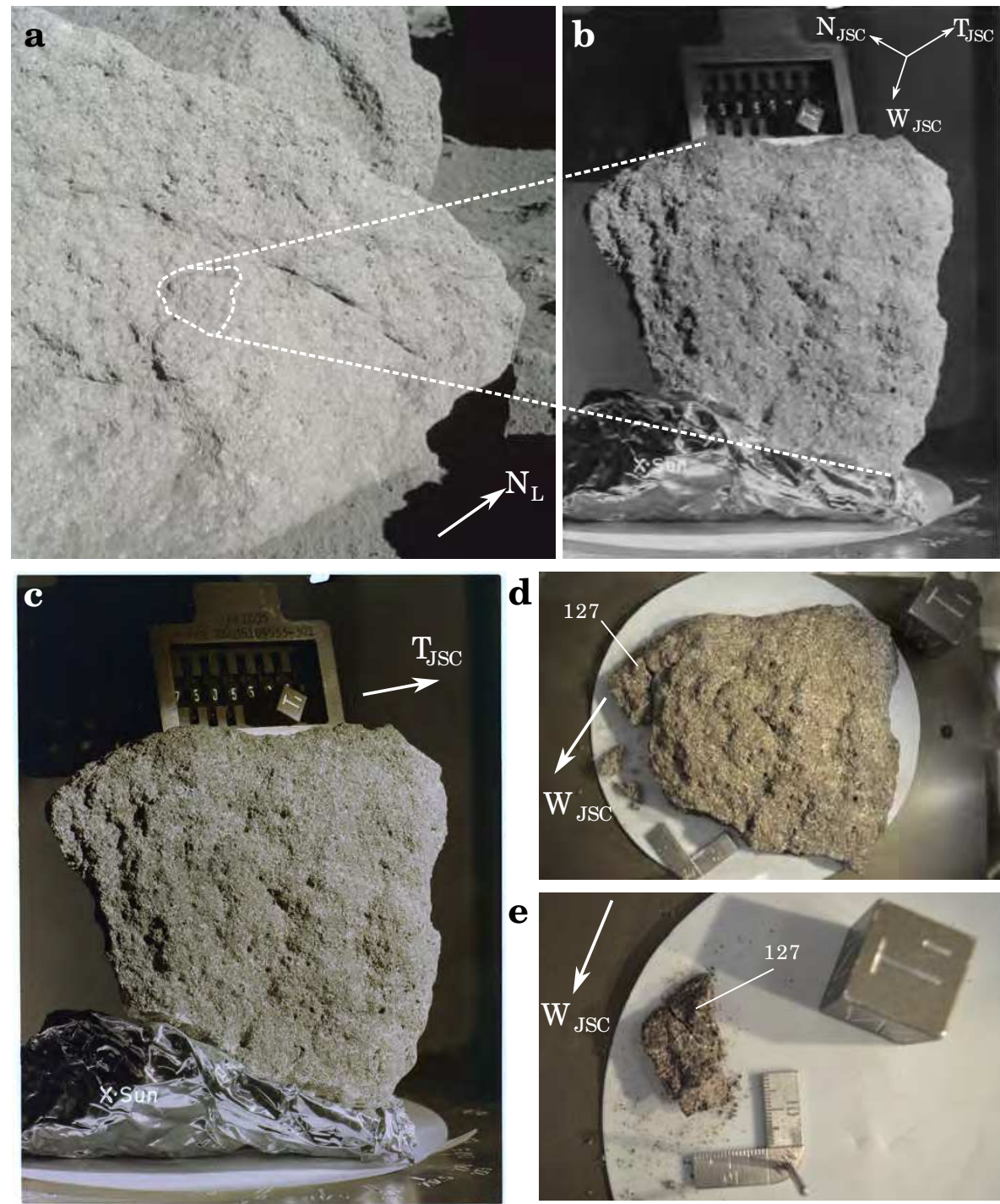

Figure S9 | The location of sample 75055 on the lunar surface and subsequent orientation and sampling of subsample 75055,127 at the JSC. (a) Astronaut photograph AS17-145-22149 showing the location of 75055 prior to sampling from the boulder on the lunar surface. (b) JSC photograph S-73-17796. (c) JSC photograph S73-17796 showing the top face of 75055 (in JSC coordinates) prior to chipping. The sample is photographed in the same orientation and lighting conditions as when sampled on the Moon. The sample cube is $1 \mathrm{~cm}$ across. (d) Photograph taken during sampling by B.P. Weiss and H.H. Schmitt in 2016 at JSC. Sample 75055 was chipped into smaller fragments. Subsample 75055,127 is in the upper left of the photo. The orientation cube is $2.5 \mathrm{~cm}$ across. (e) Photograph taken during sampling by B. Weiss and H. Schmitt in 2016 at JSC. The relative orientation of subsample 75055,127 to 75055,128 and the orientation cube $(2.5 \mathrm{~cm}$ across). 


\section{S1.3 Petrographic analysis}

Plagioclase crystals typically define lineations corresponding to flow directions within bodies of magma; the average lineation typically lies approximately in the paleohorizontal plane ${ }^{87,88}$. In an attempt to identify such lineation in our samples as an independent indicator of paleohorizontality, we analysed 30- $\mu \mathrm{m}$ thin sections 75035,84 and 75055,46 in plane-polarized and cross-polarized transmitted light. Plagioclase crystals were used to assess the degree of flow alignment, since they have the most elongate morphology and their orientation can easily be constrained parallel to the direction of twinning. A histogram of directions was calculated to determine the degree of alignment of plagioclase crystals using the directionality package in the imaging software Fiji (ImageJ) ${ }^{89,90}$. Crystal orientations were grouped in $10^{\circ}$ bins.

We analysed the same thin sections using backscattered electron microscopy (BSEM). Samples were carbon coated and imaged at the MIT Electron Microprobe Facility using a JEOL-JXA-8200 electron microprobe. Mineral compositions were assessed using wavelength dispersive spectroscopy (WDS) for $\mathrm{Fe}, \mathrm{Ni}$ and S (Table S3).
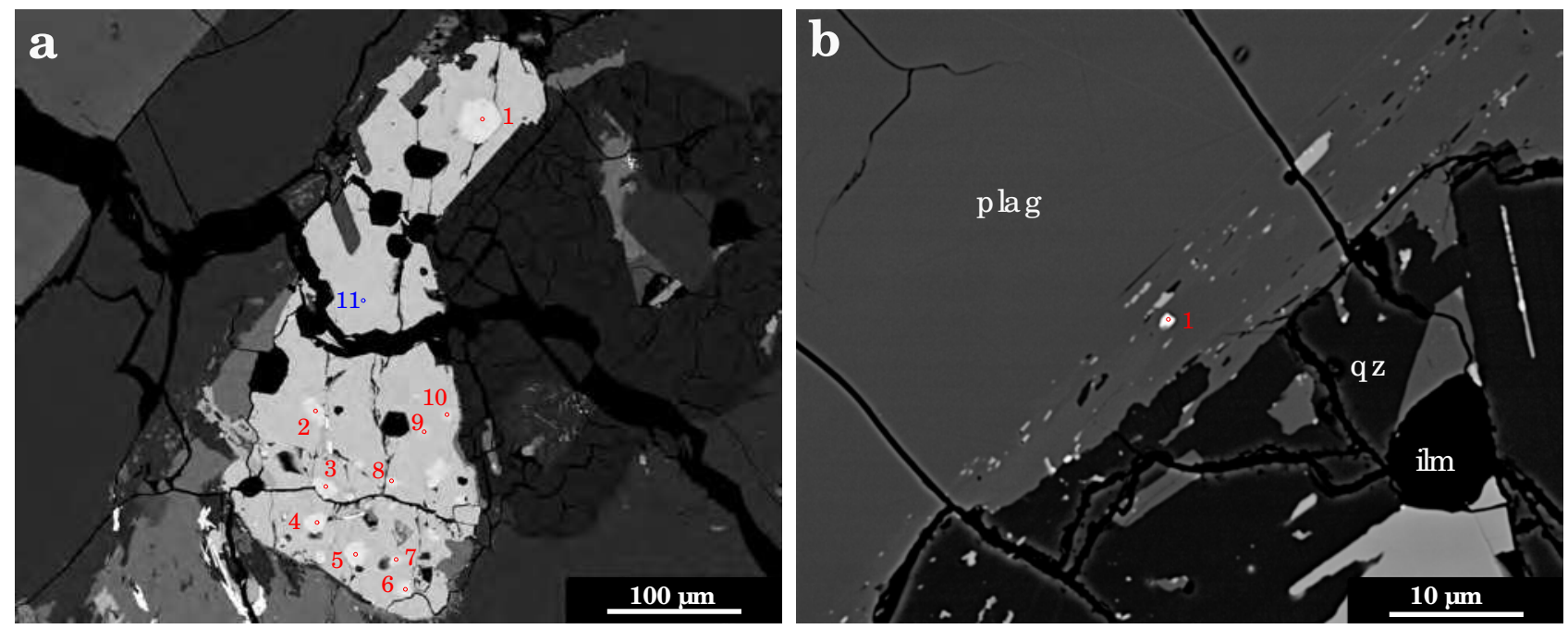

Figure S10 | BSEM images of 75035. Red (kamacite) and blue (troilite) circles/numbers show locations of WDS probe measurements (Table S3). (a) Pure Fe kamacite blebs within troilite. (b) Pure Fe kamacite bleb within a crystal of plagioclase. plag = plagioclase, qz = quartz, ilm = ilmenite. 

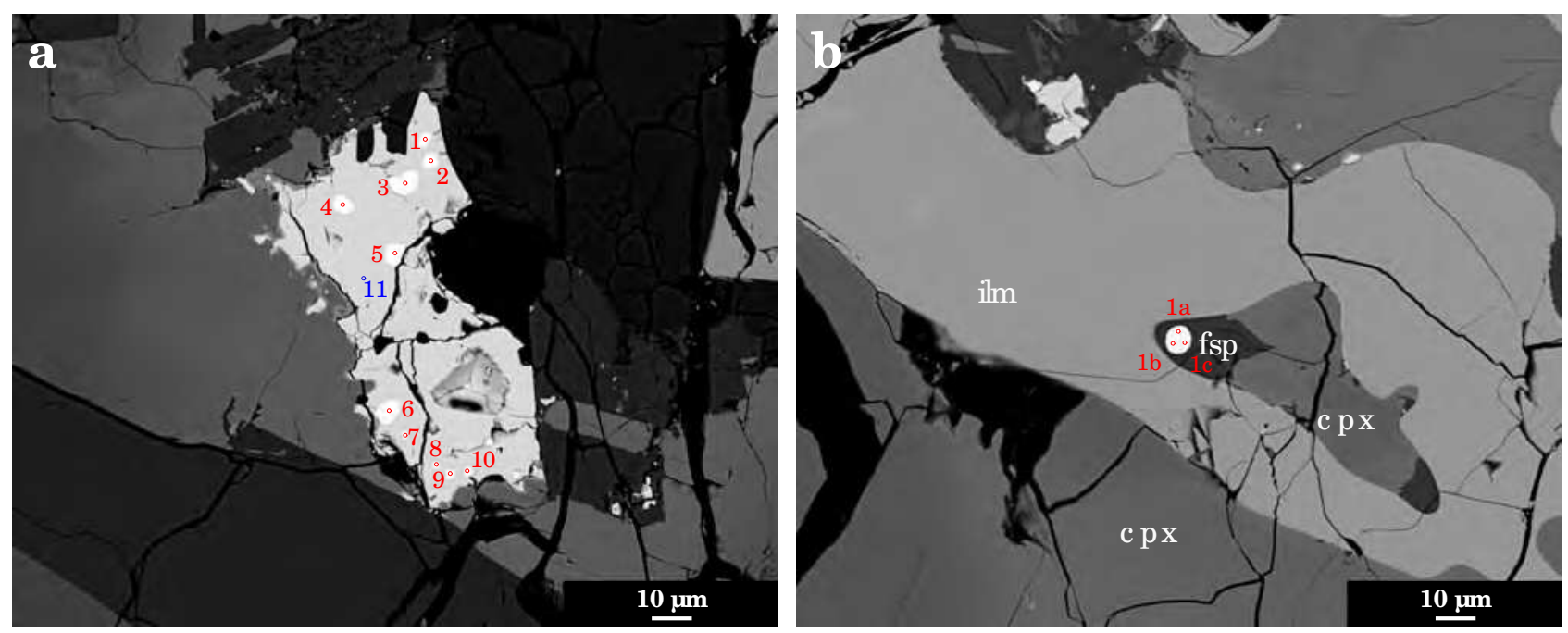

Figure S11 | BSEM images of 75055. Red (kamacite) and blue (troilite) circles/numbers show the locations of WDS probe measurements (Table S3). (a) Pure Fe kamacite blebs within troilite. (b) Pure Fe kamacite bleb within a crystal of feldspar. ilm = ilmenite, $\mathrm{cpx}=$ clinopyroxene, K-fsp $=$ feldspar.

\begin{tabular}{|c|c|c|c|c|c|c|c|c|c|c|c|}
\hline \multicolumn{6}{|c|}{75035} & \multicolumn{6}{|c|}{75055} \\
\hline Figure & Point & $\mathrm{Fe}(w t \%)$ & $\mathrm{Ni}(\mathrm{wt} \%)$ & S (wt\%) & Mineral & Figure & Point & $\mathrm{Fe}(\mathrm{wt} \%)$ & $\mathrm{Ni}(w t \%)$ & S (wt\%) & Mineral \\
\hline S10a & 1 & 100.00 & 0.00 & 0.00 & kamacite & S11a & 1 & 100.00 & 0.00 & 0.00 & kamacite \\
\hline S10a & 2 & 100.00 & 0.00 & 0.00 & kamacite & S11a & 2 & 100.00 & 0.00 & 0.00 & kamacite \\
\hline S10a & 3 & 100.00 & 0.00 & 0.00 & kamacite & S11a & 3 & 100.00 & 0.00 & 0.00 & kamacite \\
\hline S10a & 4 & 100.00 & 0.00 & 0.00 & kamacite & S11a & 4 & 100.00 & 0.00 & 0.00 & kamacite \\
\hline S10a & 5 & 100.00 & 0.00 & 0.00 & kamacite & S11a & 5 & 100.00 & 0.00 & 0.00 & kamacite \\
\hline S10a & 6 & 99.99 & 0.01 & 0.00 & kamacite & S11a & 6 & 100.00 & 0.00 & 0.00 & kamacite \\
\hline S10a & 7 & 100.00 & 0.00 & 0.00 & kamacite & S11a & 7 & 100.00 & 0.00 & 0.00 & kamacite \\
\hline S10a & 8 & 100.00 & 0.00 & 0.00 & kamacite & S11a & 8 & 100.00 & 0.00 & 0.00 & kamacite \\
\hline S10a & 9 & 100.00 & 0.00 & 0.00 & kamacite & S11a & 9 & 100.00 & 0.00 & 0.00 & kamacite \\
\hline S10a & 10 & 99.99 & 0.01 & 0.00 & kamacite & S11a & 10 & 100.00 & 0.00 & 0.00 & kamacite \\
\hline S10a & 11 & 62.38 & 0.00 & 37.62 & troilite & S11a & 11 & 62.50 & 0.00 & 37.50 & troilite \\
\hline \multirow[t]{3}{*}{ S10b } & 1 & 100.00 & 0.00 & 0.00 & kamacite & S11b & $1 a$ & 100.00 & 0.00 & 0.00 & kamacite \\
\hline & & & & & & S11b & $1 b$ & 100.00 & 0.00 & 0.00 & kamacite \\
\hline & & & & & & $\mathrm{S} 11 \mathrm{~b}$ & $1 \mathrm{c}$ & 100.00 & 0.00 & 0.00 & kamacite \\
\hline
\end{tabular}

Table S3 | WDS data for analyses of BSEM images shown in figures S10 and S11. The first and seventh columns correspond to the figure showing the location of each probe measurement for 75035 and 75055 , respectively. The second and eighth columns correspond to the specific probe point. The third and ninth columns list the Fe content, the fourth and tenth columns list the $\mathrm{Ni}$ content and the fifth and eleventh columns list the $\mathrm{S}$ content measured using WDS for 75035 and 75055 respectively. The sixth and twelfth columns list the mineral. 


\section{S2 Results}

\section{S2.1 Magnetic Carriers}

The ferromagnetic carriers in 75035 and 75055 are kamacite inclusions located in troilite and silicate minerals (e.g., plagioclase and feldspar) (Figures S10 and S11). WDS measurements of the inclusions show that the kamacite is 100 wt. \% Fe. BSEM images show that the largest inclusions are $\sim 1-10 \mu \mathrm{m}$ in diameter and have an equant morphology, suggesting they are multidomain ${ }^{91}$. Curie balance analysis showed that during heating of specimen $75055-127 \mathrm{Ac}$ the magnetization decayed as it approached $700{ }^{\circ} \mathrm{C}$, consistent with kamacite as the primary magnetic carrier. IRM acquisition suggest grains have coercivities extending to $>1000 \mathrm{mT}$, indicative of a population of smaller, single to pseudo-single domain (e.g., vortex state) kamacite grains (Figure S14).

\section{S2.2 The paleomagnetic recording fidelity of samples 75035 and 75055}

Specimens were AF demagnetized due to the likelihood of alteration during thermal demagnetization as indicated by our Curie balance analysis on specimen 75055-127Ac (Figure S12). Upon cooling, the magnetization of specimen $75055-127$ Ac increases by a factor of 3 at room temperature relative to the magnetization prior to heating. Magnetization begins to increase below $\sim 580{ }^{\circ} \mathrm{C}$ suggesting the formation of magnetite by thermal alteration. The overall increase in magnetization may be due to troilite alteration; since troilite is essentially non-magnetic, alteration to magnetite or iron will cause an increase in magnetic moment. 


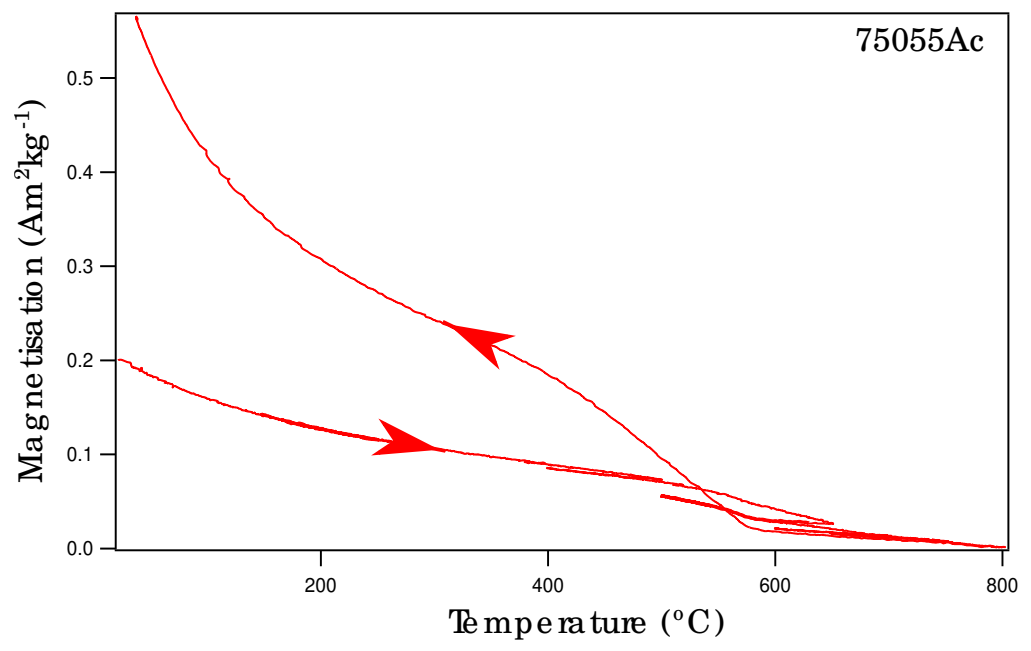

Figure S12 | Curie balance data for specimen 75055-127Ac. During heating in air to $800{ }^{\circ} \mathrm{C}$ the sample loses magnetization at $>700{ }^{\circ} \mathrm{C}$, consistent with the presence of kamacite. During cooling, the sample gains significantly more magnetization than it had prior to heating, particularly at $\sim 580^{\circ} \mathrm{C}$, suggesting magnetite has formed during thermal alteration.

To assess the paleomagnetic fidelity of the samples, we used two metrics: difference $D^{\prime}$ and error and $E<50 \%$. The metrics for our specimens are summarized in Table S4. As expected, the ability of specimens to reliably record a paleointensity diminishes with decreasing ARM strength. We found that we were able to consistently recover paleointensities of $<7.5 \mu \mathrm{T}$ from specimens of 75035 and $<37.3 \mu \mathrm{T}$ from specimens of 75055 . 


\begin{tabular}{|c|c|c|c|c|c|c|c|}
\hline Sample & $\begin{array}{l}\text { ARM DC } \\
\text { field }(\mu \mathrm{T})\end{array}$ & $\begin{array}{c}\text { TRM } \\
\text { Equivalent }(\mu \mathrm{T})\end{array}$ & $\begin{array}{c}\text { Field } \\
\text { Range (mT) }\end{array}$ & $\begin{array}{c}\text { Retrieved } \\
\text { Paleointensity }(\mu \mathbf{T})\end{array}$ & $E(\%)$ & $D^{\prime}(\%)$ & Accepted \\
\hline $75035-242 A a$ & 50 & 37.3 & $6.5-137.0$ & $27.85 \pm 4.19$ & 11 & -25 & $\checkmark$ \\
\hline \multirow[t]{3}{*}{$75035-242 \mathrm{Af}$} & 50 & 37.3 & $10.0-39.0$ & $41.24 \pm 3.43$ & 9 & 11 & $\checkmark$ \\
\hline & 20 & 14.9 & $10.0-39.0$ & $14.4 \pm 2.6$ & 17 & -4 & $\checkmark$ \\
\hline & 10 & 7.5 & $10.0-39.0$ & $15.8 \pm 2.3$ & 31 & 112 & $\times$ \\
\hline \multirow[t]{4}{*}{$75035-242 B c$} & 50 & 37.3 & $8.0-45.0$ & $48.8 \pm 3.4$ & 9 & 31 & $\checkmark$ \\
\hline & 30 & 22.4 & $8.0-45.0$ & $27.3 \pm 3.0$ & 13 & 22 & $\checkmark$ \\
\hline & 20 & 14.9 & $8.0-45.0$ & $12.3 \pm 3.2$ & 21 & -18 & $\checkmark$ \\
\hline & 10 & 7.5 & $8.0-45.0$ & $13.9 \pm 2.1$ & 28 & 86 & $\checkmark$ \\
\hline $75055-127 a$ & 20 & 14.9 & $3.0-53.0$ & $32.2 \pm 5.6$ & 38 & 116 & $x$ \\
\hline \multirow[t]{4}{*}{$75055-127 b$} & 50 & 37.3 & $4.0-24.5$ & $22.9 \pm 5.3$ & 14 & -39 & $\checkmark$ \\
\hline & 34 & 25.4 & $7.0-24.5$ & $8.5 \pm 4.9$ & 19 & -67 & $\times$ \\
\hline & 10 & 7.5 & $7.5-24.5$ & $3.8 \pm 5.4$ & 72 & -49 & $\checkmark$ \\
\hline & 5 & 3.7 & $4.0-24.5$ & $1.0 \pm 4.9$ & 131 & -73 & $\times$ \\
\hline $75055-127 \mathrm{c} 1$ & 50 & 37.3 & $0.0-50.0$ & $39.9 \pm 2.1$ & 6 & 7 & $\checkmark$ \\
\hline $75055-127 \mathrm{c} 2$ & 50 & 37.3 & $3.5-15.5$ & $24.5 \pm 3.1$ & 8 & -34 & $\checkmark$ \\
\hline $75055-127 \mathrm{Aa}$ & 50 & 37.3 & $9.0-34.0$ & $34.3 \pm 3.9$ & 10 & -8 & $\checkmark$ \\
\hline 75055-127Ab & 50 & 37.3 & $4.5-20.0$ & $33.8 \pm 4.5$ & 12 & -9 & $\checkmark$ \\
\hline $75055-127 \mathrm{Ae}$ & 50 & 37.3 & $7.5-28.0$ & $46.1 \pm 5.7$ & 15 & 24 & $\checkmark$ \\
\hline
\end{tabular}

Table S4 | Paleointensity fidelity tests for 75035 and 75055 . The first column lists the specimens. The second column lists the DC bias field used to impart an ARM. The third column lists the TRM-equivalent field using $f=1.34$. The fourth column lists the field range over which the paleointensity estimate was calculated. The fifth column lists the paleointensity calculated using the ARM paleointensity method. The sixth column lists the ratio error metric, $E$. The seventh column lists the difference metric, $D^{\prime}$. The eighth column lists whether the specimen passes the acceptance criteria defined by ref. 77 .
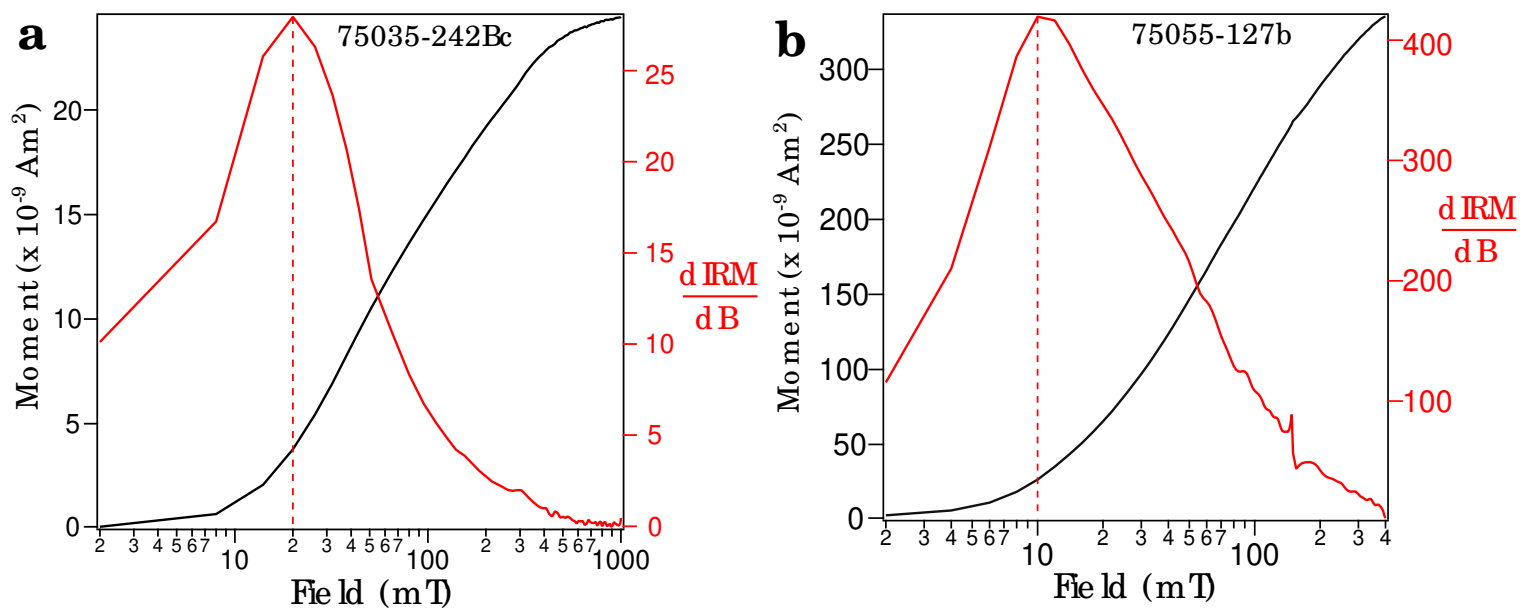

Figure S13 | IRM acquisition curves. (a) Specimen 75035-242Bc contains magnetic carriers with a peak coercivity of $\sim 20 \mathrm{mT}$ and a range of coercivities extending up to $>1000 \mathrm{mT}$. (b) Specimen 75055-127b contains magnetic carriers with a peak coercivity of $\sim 10 \mathrm{mT}$ and a range of coercivities extending up to $>400 \mathrm{mT}$. 


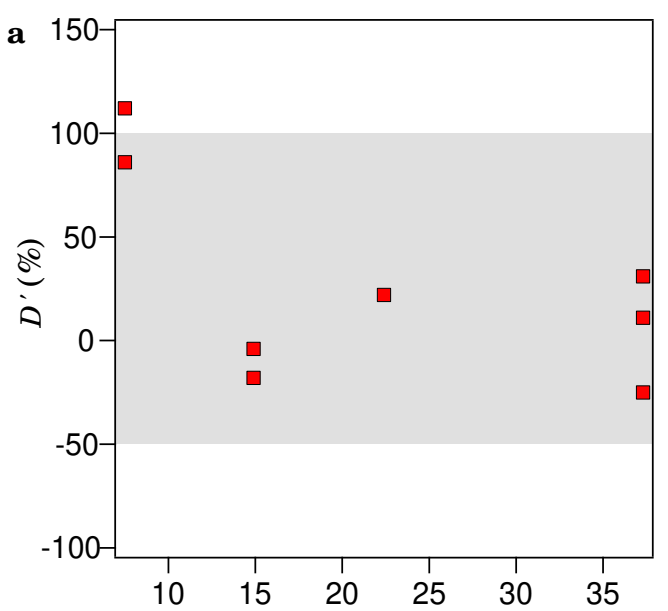

TRM-e q uiva le nt fie ld ( $\mu \mathrm{T})$

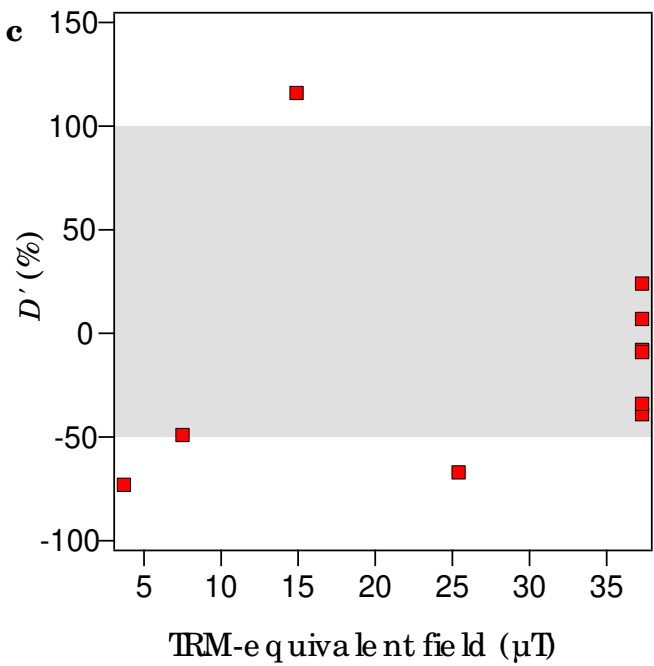

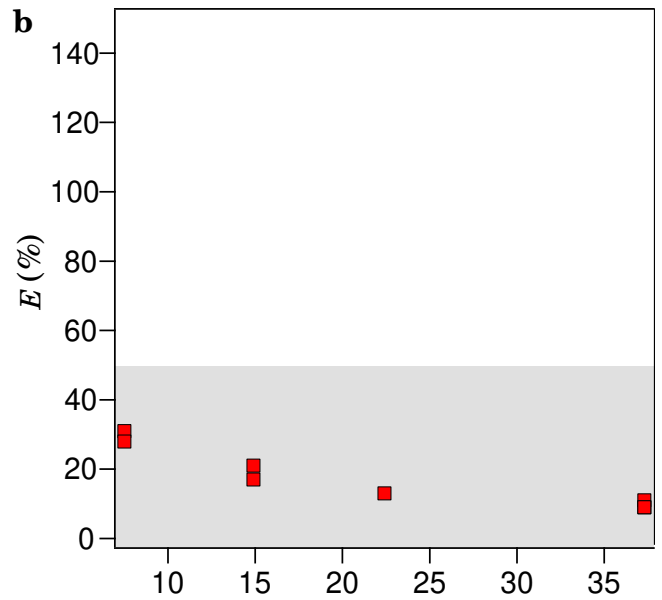

TRM-e q uiva le nt fie ld $(\mu \mathrm{T})$

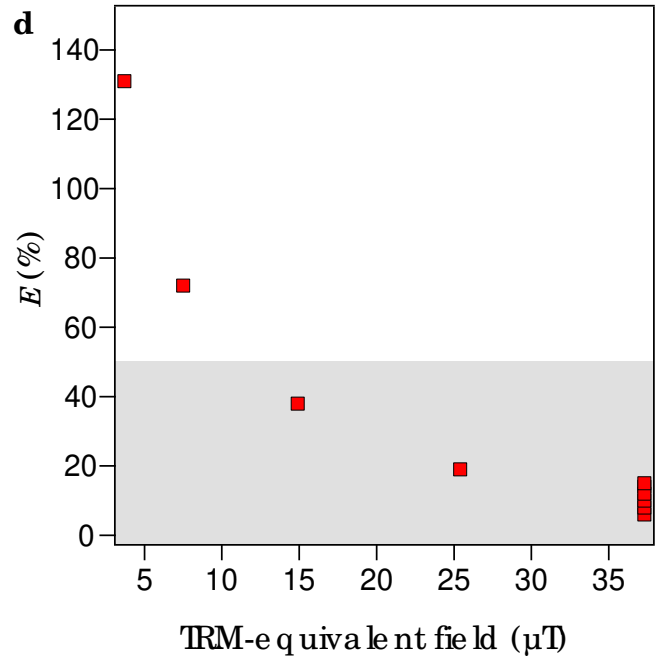

Figure S14 Paleointensity recording fidelity of 75035 and 75055 . Results are also summarized in Table S4. (a) Difference metric, $D^{\prime}$, for 75035 . The grey region $-50 \%>D^{\prime}>100 \%$ indicates samples with adequate paleomagnetic fidelity. (b) Error metric, $E^{\prime}$, for 75035 . The grey region $E<50 \%$ indicates samples with adequate paleomagnetic fidelity. (c) $D^{\prime}$ for 75055 . (d) $E$ for 75055 . 


\section{S2.3 Anisotropy of remanence and petrographic fabric analysis}

Remanence anisotropy measurements were made for two reasons: to correct the recovered paleoinclinations for anisotropy effects within each specimen and to investigate any evidence for a flow fabric in the sample defined by mutual orientation of the anisotropy axes between specimens. However, a flow fabric is not necessarily anticipated, given the predicted turbulent nature of low-viscosity lunar basaltic flows ${ }^{92,93}$. The remanence anisotropy of 7 specimens of 75035 and 6 specimens of 75055 were measured, all of which exhibited origin-trending $\mathrm{HC}$ components.

The degree of lineation $L$, foliation $F$ and anisotropy $P$ is reported for individual specimens (Tables S7 and S8). The recovered anisotropy ellipses were used to correct the NRM directions. We found that the degree of anisotropy is dependent on sample mass with smaller specimens tending to show a higher degree of anisotropy, consistent with previous mare basalt studies ${ }^{5,65}$ (Figure S16). The degree of anisotropy in our individual specimens $(P=1.0-1.5)$ is similar to that measured for terrestrial basalts $(P=1.1-1.8)^{94,95}$. In both 75035 and 75055 , there is no obvious fabric defined by the anisotropy axes of multiple specimens as indicated by the fact that Fisher dispersion factors $\kappa<4$ for each axis (Figure S15).

\begin{tabular}{|c|c|c|c|c|c|c|c|c|c|}
\hline \multirow[b]{2}{*}{ Specimen } & \multicolumn{9}{|c|}{ Direction of applied ARM } \\
\hline & 1 & 2 & 3 & 4 & 5 & 6 & 7 & 8 & 9 \\
\hline $75035-242 A a$ & $\mathrm{~T}_{\mathrm{lab}}$ & $S_{\text {lab }}$ & $\mathrm{E}_{\text {lab }}$ & $\mathrm{T}_{\mathrm{lab}}-\mathrm{S}_{\mathrm{lab}}$ & $\mathrm{B}_{\mathrm{lab}}-\mathrm{S}_{\mathrm{lab}}$ & $\mathrm{T}_{\mathrm{lab}}-\mathrm{E}_{\mathrm{lab}}$ & $\mathrm{B}_{\mathrm{lab}}-\mathrm{E}_{\mathrm{lab}}$ & $S_{l a b}-E_{l a b}$ & $N_{l a b}-E_{l a b}$ \\
\hline $75035-242 B a$ & $\mathrm{~N}_{\mathrm{lab}}$ & $\mathrm{W}_{\mathrm{lab}}$ & $\mathrm{T}_{\mathrm{lab}}$ & $\mathrm{N}_{\text {lab }}-\mathrm{W}_{\mathrm{lab}}$ & $\mathrm{S}_{\mathrm{lab}}-\mathrm{W}_{\mathrm{lab}}$ & $\mathrm{T}_{\mathrm{lab}}-\mathrm{N}_{\mathrm{lab}}$ & $\mathrm{T}_{\mathrm{lab}}-\mathrm{S}_{\mathrm{lab}}$ & $\mathrm{T}_{\mathrm{lab}}-\mathrm{W}_{\mathrm{lab}}$ & $\mathrm{T}_{\mathrm{lab}}-\mathrm{E}_{\mathrm{lab}}$ \\
\hline $75035-242 B b$ & $E_{l a b}$ & $\mathrm{~N}_{\mathrm{lab}}$ & $\mathrm{T}_{\text {lab }}$ & $N_{l a b}-E_{l a b}$ & $\mathrm{~N}_{\mathrm{lab}}-\mathrm{W}_{\mathrm{lab}}$ & $\mathrm{T}_{\mathrm{lab}}-\mathrm{E}_{\mathrm{lab}}$ & $\mathrm{T}_{\mathrm{lab}}-\mathrm{W}_{\mathrm{lab}}$ & $\mathrm{T}_{\text {lab }}-\mathrm{N}_{\mathrm{lab}}$ & $\mathrm{T}_{\mathrm{lab}}-\mathrm{S}_{\mathrm{lab}}$ \\
\hline $75035-242 B d$ & $\mathrm{~N}_{\mathrm{lab}}$ & $W_{\text {lab }}$ & $\mathrm{T}_{\text {lab }}$ & $\mathrm{N}_{\text {lab }}-\mathrm{W}_{\mathrm{lab}}$ & $\mathrm{S}_{\mathrm{lab}}-\mathrm{W}_{\mathrm{lab}}$ & $\mathrm{T}_{\text {lab }}-\mathrm{N}_{\mathrm{lab}}$ & $\mathrm{T}_{\mathrm{lab}}-\mathrm{S}_{\mathrm{lab}}$ & $\mathrm{T}_{\text {lab }}-\mathrm{W}_{\mathrm{lab}}$ & $\mathrm{T}_{\mathrm{lab}}-\mathrm{E}_{\mathrm{lab}}$ \\
\hline 75035-242Be & $\mathrm{E}_{\mathrm{lab}}$ & $\mathrm{S}_{\mathrm{lab}}$ & $\mathrm{B}_{\mathrm{lab}}$ & $S_{l a b}-E_{l a b}$ & $S_{l a b}-W_{l a b}$ & $\mathrm{~B}_{\mathrm{lab}}-\mathrm{E}_{\mathrm{lab}}$ & $\mathrm{B}_{\mathrm{lab}}-\mathrm{W}_{\mathrm{lab}}$ & $\mathrm{T}_{\text {lab }}-\mathrm{N}_{\mathrm{lab}}$ & $\mathrm{T}_{\mathrm{lab}}-\mathrm{S}_{\mathrm{lab}}$ \\
\hline $75035-242 B f$ & $E_{l a b}$ & $\mathrm{~N}_{\mathrm{lab}}$ & $\mathrm{T}_{\text {lab }}$ & $N_{l a b}-E_{l a b}$ & $\mathrm{~N}_{\text {lab }}-\mathrm{W}_{\mathrm{lab}}$ & $\mathrm{T}_{\text {lab }}-\mathrm{E}_{\mathrm{lab}}$ & $\mathrm{T}_{\text {lab }}-\mathrm{W}_{\mathrm{lab}}$ & $\mathrm{T}_{\text {lab }}-\mathrm{N}_{\mathrm{lab}}$ & $\mathrm{T}_{\mathrm{lab}}-\mathrm{S}_{\mathrm{lab}}$ \\
\hline $75035-242 B g$ & $\mathrm{~N}_{\mathrm{lab}}$ & $W_{\text {lab }}$ & $\mathrm{T}_{\text {lab }}$ & $\mathrm{N}_{\mathrm{lab}}-\mathrm{W}_{\mathrm{lab}}$ & $S_{l a b}-W_{l a b}$ & $\mathrm{~T}_{\mathrm{lab}}-\mathrm{N}_{\mathrm{lab}}$ & $\mathrm{T}_{\mathrm{lab}}-\mathrm{S}_{\mathrm{lab}}$ & $\mathrm{T}_{\mathrm{lab}}-\mathrm{W}_{\mathrm{lab}}$ & $T_{\text {lab }}-E_{\text {lab }}$ \\
\hline $75055-127 \mathrm{Aa}$ & $\mathrm{T}_{\mathrm{lab}}$ & $\mathrm{S}_{\mathrm{lab}}$ & $\mathrm{E}_{\mathrm{lab}}$ & $\mathrm{T}_{\text {lab }}-\mathrm{S}_{\mathrm{lab}}$ & $\mathrm{B}_{\mathrm{lab}}-\mathrm{S}_{\mathrm{lab}}$ & $\mathrm{T}_{\text {lab }}-\mathrm{E}_{\mathrm{lab}}$ & $\mathrm{B}_{\mathrm{lab}}-\mathrm{E}_{\mathrm{lab}}$ & $S_{l a b}-E_{l a b}$ & $\mathrm{~N}_{\mathrm{lab}}-\mathrm{E}_{\mathrm{lab}}$ \\
\hline $75055-127 A b$ & $\mathrm{~T}_{\mathrm{lab}}$ & $S_{\text {lab }}$ & $E_{\text {lab }}$ & $\mathrm{T}_{\mathrm{lab}}-\mathrm{S}_{\mathrm{lab}}$ & $\mathrm{B}_{\mathrm{lab}}-\mathrm{S}_{\mathrm{lab}}$ & $\mathrm{T}_{\mathrm{lab}}-\mathrm{E}_{\mathrm{lab}}$ & $\mathrm{B}_{\mathrm{lab}}-\mathrm{E}_{\mathrm{lab}}$ & $S_{l a b}-E_{l a b}$ & $N_{l a b}-E_{l a b}$ \\
\hline $75055-127 A c$ & $\mathrm{~T}_{\mathrm{lab}}$ & $\mathrm{E}_{\mathrm{lab}}$ & $\mathrm{N}_{\mathrm{lab}}$ & $\mathrm{T}_{\mathrm{lab}}-\mathrm{E}_{\mathrm{lab}}$ & $\mathrm{B}_{\mathrm{lab}}-\mathrm{E}_{\mathrm{lab}}$ & $\mathrm{T}_{\mathrm{lab}}-\mathrm{N}_{\mathrm{lab}}$ & $\mathrm{B}_{\mathrm{lab}}-\mathrm{N}_{\mathrm{lab}}$ & $\mathrm{N}_{\mathrm{lab}}-\mathrm{E}_{\mathrm{lab}}$ & $\mathrm{N}_{\mathrm{lab}}-\mathrm{W}_{\mathrm{lab}}$ \\
\hline $75055-127 \mathrm{Ae}$ & $\mathrm{N}_{\text {lab }}$ & $W_{\text {lab }}$ & $\mathrm{T}_{\text {lab }}$ & $N_{l a b}-W_{l a b}$ & $S_{l a b}-W_{l a b}$ & $\mathrm{~T}_{\text {lab }}-\mathrm{N}_{\text {lab }}$ & $\mathrm{T}_{\text {lab }}-\mathrm{S}_{\mathrm{lab}}$ & $\mathrm{T}_{\mathrm{lab}}-\mathrm{W}_{\mathrm{lab}}$ & $\mathrm{T}_{\mathrm{lab}}-\mathrm{E}_{\mathrm{lab}}$ \\
\hline $75055-127 a$ & $\mathrm{~T}_{\text {lab }}$ & $\mathrm{N}_{\mathrm{lab}}$ & $\mathrm{W}_{\mathrm{lab}}$ & $\mathrm{T}_{\text {lab }}-\mathrm{N}_{\mathrm{lab}}$ & $\mathrm{B}_{\mathrm{lab}}-\mathrm{N}_{\mathrm{lab}}$ & $\mathrm{T}_{\text {lab }}-\mathrm{W}_{\mathrm{lab}}$ & $\mathrm{B}_{\mathrm{lab}}-\mathrm{W}_{\mathrm{lab}}$ & $N_{l a b}-W_{l a b}$ & $S_{l a b}-W_{l a b}$ \\
\hline $75055-127 b$ & $\mathrm{~T}_{\text {lab }}$ & $\mathrm{N}_{\mathrm{lab}}$ & $\mathrm{W}_{\mathrm{lab}}$ & $\mathrm{T}_{\text {lab }}-\mathrm{N}_{\text {lab }}$ & $\mathrm{B}_{\mathrm{lab}}-\mathrm{N}_{\mathrm{lab}}$ & $\mathrm{T}_{\mathrm{lab}}-\mathrm{W}_{\mathrm{lab}}$ & $\mathrm{B}_{\mathrm{lab}}-\mathrm{W}_{\mathrm{lab}}$ & $N_{l a b}-W_{l a b}$ & $S_{\text {lab }}-W_{\text {lab }}$ \\
\hline
\end{tabular}

Table S5 | For AARM measurements, ARMs were applied in nine directions (shown by columns $1-9)$ corresponding to the anisotropy matrix. Specimens were mounted in different orientations with respect to laboratory coordinates and reconstructed to a mutual orientation in JSC coordinates after measuring. The demagnetization procedure between each ARM step is shown in Table S6. 


\begin{tabular}{cccc}
\hline $\begin{array}{c}\text { ARM } \\
\text { direction }\end{array}$ & $\begin{array}{c}\text { Demagnetization } \\
\text { direction 1 }\end{array}$ & $\begin{array}{c}\text { Demagnetization } \\
\text { direction 2 }\end{array}$ & $\begin{array}{c}\text { Demagnetization } \\
\text { direction 3 }\end{array}$ \\
\hline$E_{\text {mag }}$ & $\mathrm{N}_{\text {mag }}$ & $\mathrm{T}_{\text {mag }}$ & $\mathrm{E}_{\text {mag }}$ \\
$\mathrm{N}_{\text {mag }}$ & $\mathrm{E}_{\text {mag }}$ & $\mathrm{T}_{\text {mag }}$ & $\mathrm{N}_{\text {mag }}$ \\
$\mathrm{T}_{\text {mag }}$ & $\mathrm{N}_{\text {mag }}$ & $\mathrm{E}_{\text {mag }}$ & $\mathrm{T}_{\text {mag }}$ \\
$\mathrm{NE}_{\text {mag }}$ & $\mathrm{T}_{\text {mag }}$ & $\mathrm{NW}_{\text {mag }}$ & $\mathrm{N} E_{\text {mag }}$ \\
$\mathrm{NW}_{\text {mag }}$ & $\mathrm{NE}_{\text {mag }}$ & $\mathrm{T}_{\text {mag }}$ & $\mathrm{NW_{ \text {mag } }}$ \\
$\mathrm{TE}_{\text {mag }}$ & $\mathrm{N}_{\text {mag }}$ & $\mathrm{TW}_{\text {mag }}$ & $\mathrm{TE}_{\text {mag }}$ \\
$\mathrm{TW}_{\text {mag }}$ & $\mathrm{T} E_{\text {mag }}$ & $\mathrm{N}_{\text {mag }}$ & $\mathrm{TW}_{\text {mag }}$ \\
$\mathrm{TN}_{\text {mag }}$ & $\mathrm{E}_{\text {mag }}$ & $\mathrm{TS}$ & $\mathrm{T}$ \\
$\mathrm{TS}_{\text {mag }}$ & $\mathrm{TN}_{\text {mag }}$ & $\mathrm{E}_{\text {mag }}$ & $\mathrm{TS}_{\text {mag }}$ \\
\hline
\end{tabular}

Table S6 | The orientation of the applied ARM and the three subsequent demagnetization steps used for AARM experiments. The first column shows the direction in which the ARM was applied. Samples were then demagnetized in the three orientations listed in the second, third and fourth columns, respectively.

\begin{tabular}{cccccccc}
\hline & $\mathbf{7 5 0 3 5 - 2 4 2 A a}$ & $\mathbf{7 5 0 3 5 - 2 4 2 B a}$ & $\mathbf{7 5 0 3 5 - 2 4 2 B b}$ & $\mathbf{7 5 0 3 5 - 2 4 2 B d}$ & $\mathbf{7 5 0 3 5 - 2 4 2 B e}$ & $\mathbf{7 5 0 3 5 - 2 4 2 B f}$ & $\mathbf{7 5 0 3 5 - 2 4 2 B g}$ \\
\hline$p_{1}$ & 658 & 998 & 1169 & 310 & 1404 & 1096 & 1117 \\
$\operatorname{dec}\left({ }^{\circ}\right)$ & 107 & 075 & 080 & 099 & 065 & 232 & 278 \\
$\operatorname{inc}\left({ }^{\circ}\right)$ & -23 & -37 & -46 & -23 & -05 & -42 & -03 \\
$p_{2}$ & 502 & 940 & 1140 & 252 & 1183 & 1066 & 838 \\
$\operatorname{dec}\left({ }^{\circ}\right)$ & 040 & 075 & 329 & 033 & 147 & 319 & 024 \\
$\operatorname{inc}\left({ }^{\circ}\right)$ & 42 & 53 & -20 & 43 & 60 & 04 & -78 \\
$p_{3}$ & 471 & 783 & 1038 & 229 & 1125 & 1054 & 731 \\
$\operatorname{dec}\left({ }^{\circ}\right)$ & 358 & 345 & 043 & 349 & 338 & 045 & 008 \\
$\operatorname{inc}\left({ }^{\circ}\right)$ & -40 & 00 & 38 & -39 & 31 & -47 & 12 \\
$L$ & 1.309 & 1.062 & 1.025 & 1.227 & 1.187 & 1.028 & 1.333 \\
$F$ & 1.067 & 1.200 & 1.098 & 1.102 & 1.051 & 1.011 & 1.146 \\
$P$ & 1.397 & 1.275 & 1.125 & 1.353 & 1.248 & 1.040 & 1.528 \\
Mass $(\mathrm{mg})$ & 59.8 & 64.8 & 101.9 & 16.1 & 132.1 & 95.6 & 74.2 \\
\hline
\end{tabular}

Table S7 | AARM results for specimens of 75035. The first, second and third rows are the magnitude, declination and inclination (in JSC coordinates) of the maximum principal anisotropy axis $p_{1}$. The fourth, fifth and sixth rows are the magnitude, declination and inclination of the intermediate principal anisotropy axis, $p_{2}$. The seventh, eighth and ninth rows are the magnitude, declination and inclination of the minimum principal anisotropy axis, $p_{3}$. The directions of the principal axes for each specimen are plotted in Figure S15a. The tenth row is $L$, is the degree of lineation, the eleventh row is $F$, the degree of foliation and the twelfth row is $P$, the degree of anisotropy. The thirteenth row is the mass of the specimen. 


\begin{tabular}{ccccccc}
\hline & 75055-127Aa & 75055-127Ab & 75055-127Ac & 75055-127Ae & 75055-127a & 75055-127b \\
\hline$p_{1}$ & 1197 & 710 & 587 & 539 & 2705 & 4931 \\
$\operatorname{dec}\left({ }^{\circ}\right)$ & 221 & 325 & 305 & 224 & 032 & 321 \\
$\operatorname{inc}\left({ }^{\circ}\right)$ & -11 & -19 & -16 & -24 & 04 & 18 \\
$p_{2}$ & 1160 & 641 & 586 & 537 & 2374 & 4830 \\
$\operatorname{dec}\left({ }^{\circ}\right)$ & 315 & 051 & 021 & 240 & 282 & 074 \\
$\operatorname{inc}\left({ }^{\circ}\right)$ & -17 & 11 & 38 & 64 & 77 & 49 \\
$p_{3}$ & 1017 & 512 & 499 & 514 & 2364 & 4656 \\
$\operatorname{dec}\left({ }^{\circ}\right)$ & 281 & 291 & 233 & 316 & 123 & 038 \\
$\operatorname{inc}\left({ }^{\circ}\right)$ & 68 & 66 & 47 & -06 & 12 & -34 \\
$L$ & 1.031 & 1.107 & 1.001 & 1.004 & 1.139 & 1.020 \\
$F$ & 1.140 & 1.251 & 1.173 & 1.043 & 1.004 & 1.037 \\
$P$ & 1.176 & 1.386 & 1.175 & 1.048 & 1.144 & 1.059 \\
Mass $(\mathrm{mg})$ & 102.5 & 62.6 & 58.3 & 54.9 & 271.0 & 641.0 \\
\hline
\end{tabular}

Table S8 | Anisotropy of ARM results for specimens of 75055. The first, second and third rows are the magnitude, declination and inclination (in JSC coordinates) of the maximum principal anisotropy axis $p_{1}$. The fourth, fifth and sixth rows are the magnitude, declination and inclination of the intermediate principal anisotropy axis, $p_{2}$. The seventh, eighth and ninth rows are the magnitude, declination and inclination of the minimum principal anisotropy axis, $p_{3}$. The directions of the principal axes for each specimen are plotted in Figure S15b. The tenth row is $L$, is the degree of lineation, the eleventh row is $F$, the degree of foliation and the twelfth row is $P$, the degree of anisotropy. The thirteenth row is the mass of the specimen.

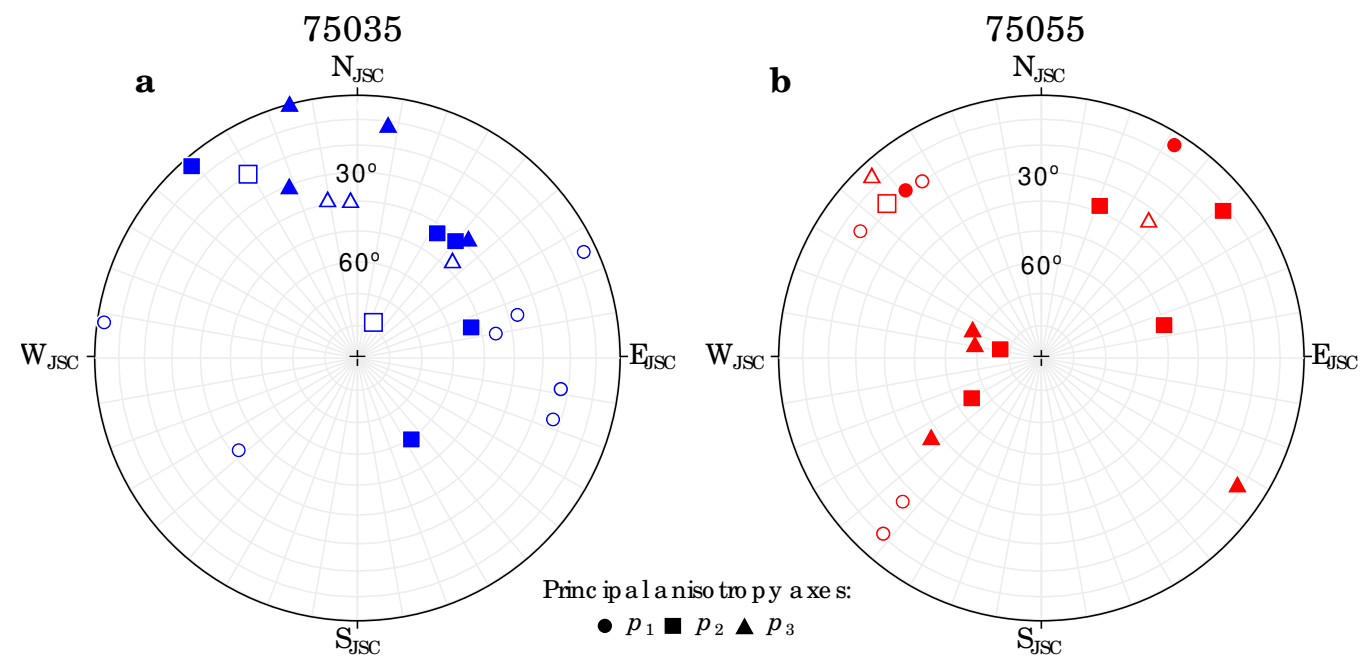

Figure S15 | Equal area polar stereonet projections showing the minimum $p_{3}$ (triangles), intermediate $p_{2}$ (squares) and maximum $p_{1}$ (circles) principal anisotropy axes for specimens of (a) 75035 and (b) 75055 in JSC coordinates. Solid symbols are in the lower hemisphere, open symbols are in the upper hemisphere. There is no macro clear fabric defined by the anisotropy axes, Fisher precision parameters are $<4$ for all axes. 


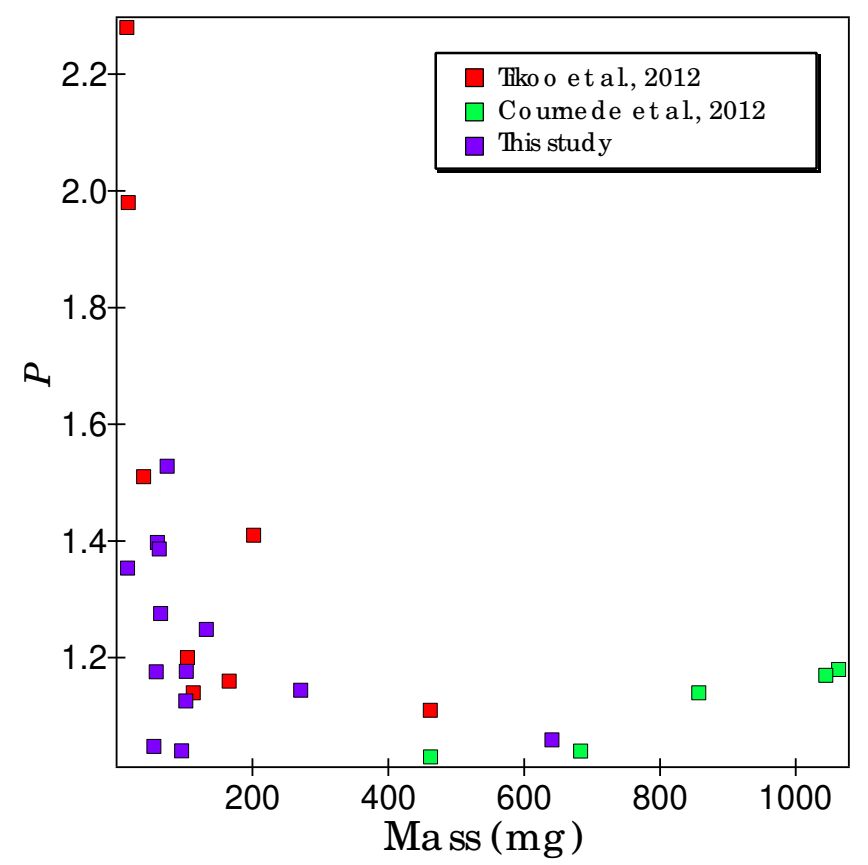

Figure S16 | The degree of anisotropy, $P$, recovered for AARM measurements as a function of sample mass. Our results are consistent with previous studies of lunar basalts conducted by ref. 5 and ref. 65.

Hand samples and thin sections were also examined for evidence of any flow fabrics. Rose diagrams of plagioclase crystal orientations were found to have a circular variance of 0.73 and 0.98 for 75035 and 75055 , respectively, where the circular variance is 0 for perfect alignment in one direction and 1 for an entirely uniform distribution (Figure S17). The lack of fabric defined within the mineralogy of the samples is consistent with the fact that well-aligned fabrics only develop in laminar flows, whereas the flow of lunar basalts is expected to be turbulent ${ }^{92,93}$. 

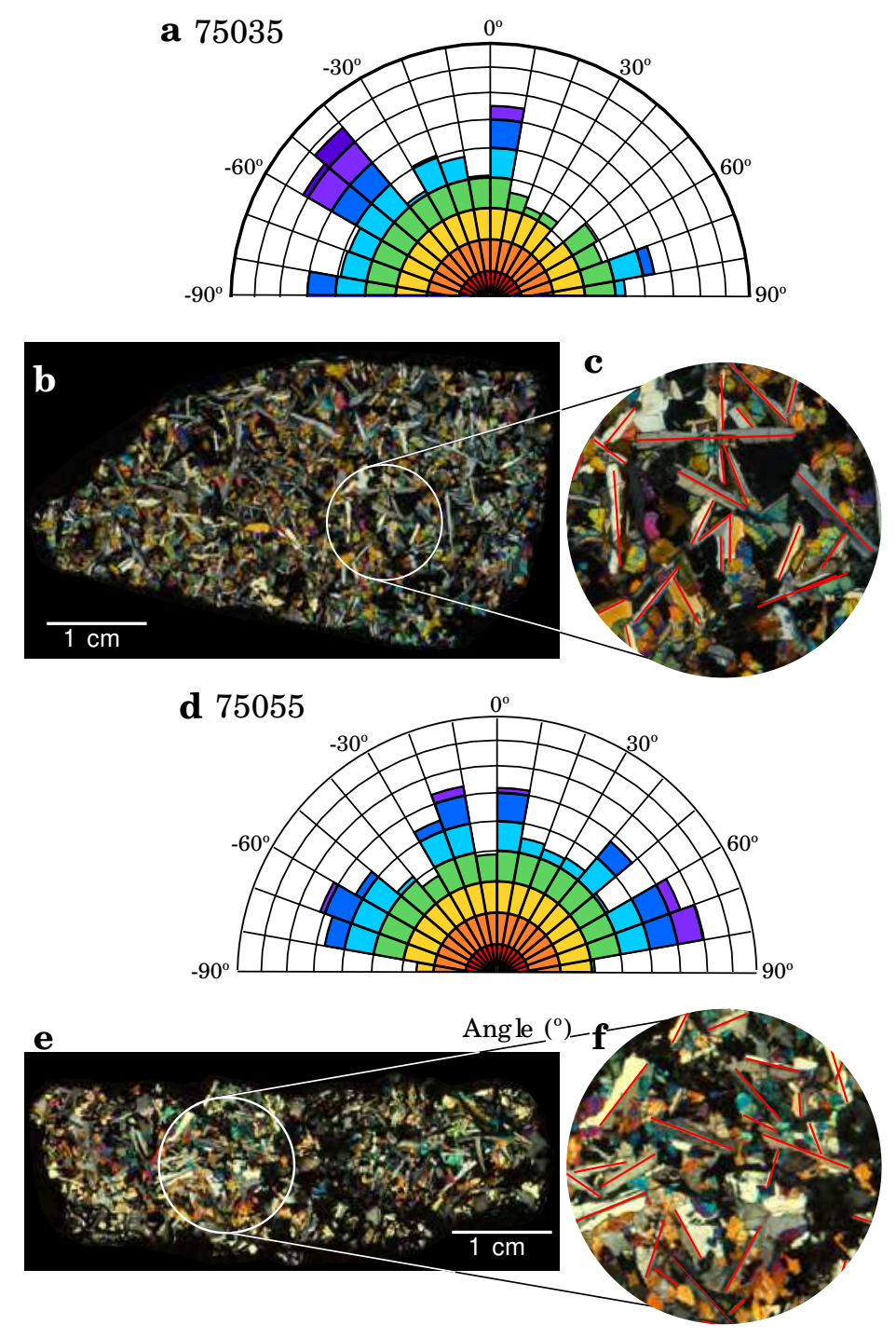

Figure S17 | Analysis of the orientation of plagioclase crystals assessed from photomicrographs of thin sections 75035,84 and 75055,46 . (a) Equal area rose diagram of plagioclase orientation distributions for 75035. The circular variance of the distribution is 0.73 . (b) Cross-polarized transmitted light image of thin section 75035. (c) Magnified region of thin section 75035. Red lines show the identified plagioclase orientations used for orientation analysis. (d) Equal area rose diagram of plagioclase orientation distributions for 75055 . The circular variance of the distribution is 0.98. (e) and (f) are the same as (b) and (c) for 75055 . 


\begin{tabular}{|c|c|c|}
\hline Specimen & Experiment & Paleointensity $(\mu \mathbf{T})$ \\
\hline $75035-242 A a$ & $100 \mu$ T ARM & $33.1 \pm 5.5$ \\
\hline $75035-242 \mathrm{Ae}$ & $100 \mu$ T ARM & $101.3 \pm 7.9$ \\
\hline 75035-242Af & $100 \mu$ T ARM & $23.7 \pm 2.4$ \\
\hline \multirow[t]{2}{*}{$75035-242 \mathrm{BC}$} & $100 \mu$ T ARM & $47.7 \pm 4.7$ \\
\hline & Mean $\pm 1 \sigma$ & $50.7 \pm 13.5$ \\
\hline $75055-127 a$ & $50 \mu$ T ARM & $18.2 \pm 5.7$ \\
\hline $75055-127 b$ & $100 \mu$ T ARM & $50.0 \pm 10.6$ \\
\hline $75055-127 \mathrm{c} 1$ & $200 \mu$ T ARM & $50.4 \pm 5.2$ \\
\hline $75055-127 \mathrm{c} 2$ & $200 \mu$ T ARM & $84.3 \pm 11.9$ \\
\hline 75055-127Aa & $100 \mu \mathrm{T}$ ARM & $33.3 \pm 2.6$ \\
\hline 75055-127Ab & $100 \mu$ T ARM & $12.2 \pm 8.3$ \\
\hline $75055-127 \mathrm{AC}$ & $100 \mu \mathrm{T}$ ARM & $34.4 \pm 4.2$ \\
\hline \multirow[t]{2}{*}{ 75055-127Ae } & $100 \mu \mathrm{T}$ ARM & $173.1 \pm 14.3$ \\
\hline & Mean $\pm 1 \sigma$ & $57.0 \pm 17.3$ \\
\hline
\end{tabular}

Table S9 | Paleointensity estimates for HC components for samples 75035 and 75055 . The first column is the measured specimen. The second column shows the strength of the applied ARM used for the paleointensity estimate. The third column shows the estimated paleointensity and $2 \sigma$ uncertainty calculated from a two-tailed Student's $t$ test but does not take into account the factor of 5 uncertainty associated with the calibration factor $f^{\prime 1}$. Paleointensities were calculated over the HC field range shown in Table S10. 


\begin{tabular}{|c|c|c|c|c|c|c|c|c|c|c|c|c|c|c|}
\hline \multirow[t]{2}{*}{ Specimen } & \multirow{2}{*}{$\begin{array}{l}\text { Mass } \\
(\mathrm{mg})\end{array}$} & \multirow{2}{*}{$\begin{array}{c}\text { Magnetization } \\
\left(\times \mathbf{1 0}^{-6} \mathbf{A m}^{2} \mathbf{k g}^{-1}\right)\end{array}$} & \multicolumn{7}{|c|}{ HC component } & \multicolumn{5}{|c|}{ LC component } \\
\hline & & & $\operatorname{Dec}\left(\left(^{\circ}\right)\right.$ & $\operatorname{Inc}\left({ }^{\circ}\right)$ & $\operatorname{MAD}\left({ }^{\circ}\right)$ & $\operatorname{DANG}\left({ }^{\circ}\right)$ & $\mathrm{AF}(\mathrm{mT})$ & O-trending? & Anchored? & $\operatorname{Dec}\left({ }^{\circ}\right)$ & $\operatorname{Inc}\left({ }^{\circ}\right)$ & $\operatorname{MAD}\left({ }^{\circ}\right)$ & $\operatorname{DANG}\left({ }^{\circ}\right)$ & $\mathrm{AF}(\mathrm{mT})$ \\
\hline $75035-242 \mathrm{Aa}$ & 59.8 & 2.78 & 354 & -66 & 23 & 155 & $0-5$ & & & & & & & \\
\hline $75035-242 A b$ & 203.1 & 0.64 & & & & & & & & & & & & \\
\hline $75035-242 A C$ & 232.8 & 2.17 & & & & & & & & 358 & 77 & 28 & 72 & $0-6.5$ \\
\hline $75035-242 A d^{*}$ & 322.3 & 7.45 & 324 & 78 & 5 & 0 & $17-28$ & $\checkmark$ & $\checkmark$ & 245 & 42 & 6 & 37 & $0-7$ \\
\hline $75035-242 \mathrm{Ae}$ & 46.6 & 17.30 & 93 & 45 & 7 & 0 & $11.5-47$ & $\checkmark$ & $\checkmark$ & 69 & 31 & 11 & 14 & $0-11$ \\
\hline $75035-242 \mathrm{Af}$ & 188 & 1.99 & 82 & 44 & 26 & 0 & $10-60$ & $\checkmark$ & $\checkmark$ & 191 & 16 & 29 & 96 & $0-10$ \\
\hline $75035-242 \mathrm{Ag}$ & 169.3 & 1.46 & & & & & & & & 205 & 58 & 17 & 24 & $0-3.5$ \\
\hline $75035-242 A h^{*}$ & 277.8 & 9.83 & 185 & 83 & 8 & 0 & $15-50$ & $\checkmark$ & $\checkmark$ & 222 & 32 & 6 & 38 & $0-7$ \\
\hline $75035-242 B a$ & 64.9 & 8.03 & 85 & 58 & 8 & 0 & $11-33$ & $\checkmark$ & $\checkmark$ & 86 & -49 & 11 & 93 & $0-5$ \\
\hline $75035-242 B b$ & 102 & 4.48 & & & & & & & & 102 & 70 & 15 & 14 & $0-14$ \\
\hline $75035-242 \mathrm{BC}$ & 65.3 & 6.34 & 107 & 54 & 11 & 0 & $10.5-40$ & $\checkmark$ & $\checkmark$ & 345 & -32 & 37 & 137 & $0-7$ \\
\hline $75035-242 B d$ & 16.1 & 12.00 & 94 & 59 & 44 & 47 & $10-40$ & $x$ & $x$ & 105 & 42 & 27 & 27 & $0-7.5$ \\
\hline $75035-242 B e$ & 132.1 & 4.44 & 52 & 16 & 16 & 0 & $10-40$ & $\checkmark$ & $\checkmark$ & 58 & 41 & 14 & 9 & $0-6$ \\
\hline $75035-242 B f$ & 95.6 & 6.54 & 94 & 24 & 7 & 0 & $15-50$ & $\checkmark$ & $\checkmark$ & 178 & -53 & 20 & 110 & $0-3.5$ \\
\hline $75035-242 B g$ & 74.2 & 6.31 & 94 & 40 & 14 & 0 & $16-45$ & $\checkmark$ & $\checkmark$ & 39 & 62 & 19 & 33 & $0-11$ \\
\hline 75055-127a & 271 & 4.03 & 210 & 25 & 20 & 27 & $2.5-24$ & $x$ & $x$ & 353 & 48 & 10 & 103 & $0-2$ \\
\hline $75055-127 b$ & 641 & 3.95 & 214 & 8 & 10 & 0 & $27-49$ & $\checkmark$ & $\checkmark$ & 37 & 49 & 5 & 126 & $0-1.5$ \\
\hline $75055-127 \mathrm{c} 1$ & 156 & 4.03 & 221 & 14 & 8 & 0 & $4-35$ & $\checkmark$ & $\checkmark$ & 294 & 39 & 30 & 70 & $0-1.5$ \\
\hline $75055-127 \mathrm{c} 2$ & 166 & 7.29 & 215 & 1 & 6 & 0 & $10-35$ & $\checkmark$ & $\checkmark$ & 341 & -20 & 34 & 131 & $0-9.5$ \\
\hline $75055-127 \mathrm{Aa}$ & 102.5 & 14.60 & 225 & 26 & 9 & 0 & $2.5-45$ & $\checkmark$ & $\checkmark$ & 183 & 2 & 4 & 44 & $0-2$ \\
\hline $75055-127 \mathrm{Ab}$ & 62.6 & 4.15 & & & & & & & & & & & & \\
\hline $75055-127 \mathrm{AC}$ & 54.9 & 6.19 & 205 & 13 & 13 & 0 & $4.5-39$ & $\checkmark$ & $\checkmark$ & 89 & -48 & 20 & 124 & $0-4$ \\
\hline $75055-127 A d$ & 8.3 & 3.25 & & & & & & & & & & & & \\
\hline $75055-127 \mathrm{Ae}$ & 58.3 & 20.60 & 219 & 8 & 7 & 14 & $7.5-55$ & $x$ & $x$ & 92 & -66 & 27 & 101 & $0-6.5$ \\
\hline
\end{tabular}

Table S10 | LC and HC components identified during AF demagnetization of specimens of 75035 and 75055 . Declinations and inclinations are in laboratory coordinates. In the case where no reasonable fit could be made, the row is left blank. The first column lists the specimen. The second column lists the mass of the specimen and the third lists the specific magnetization of the NRM. Then for each component the columns are as follows: the first and second columns are declination and inclination, respectively; the third column lists the MAD (after anchoring to the origin for $\mathrm{HC}$ components found to be origin-trending); the fourth column lists the deviation angle; the fifth column lists the AF range over which the component was fitted. For the HC component, the sixth column lists whether the component was statistically origin-trending (MAD>DANG) and the seventh column lists whether the fit was anchored to the origin. Italicized subsamples were used to calculate mean $\mathrm{HC}$ directions and paleointensities. 

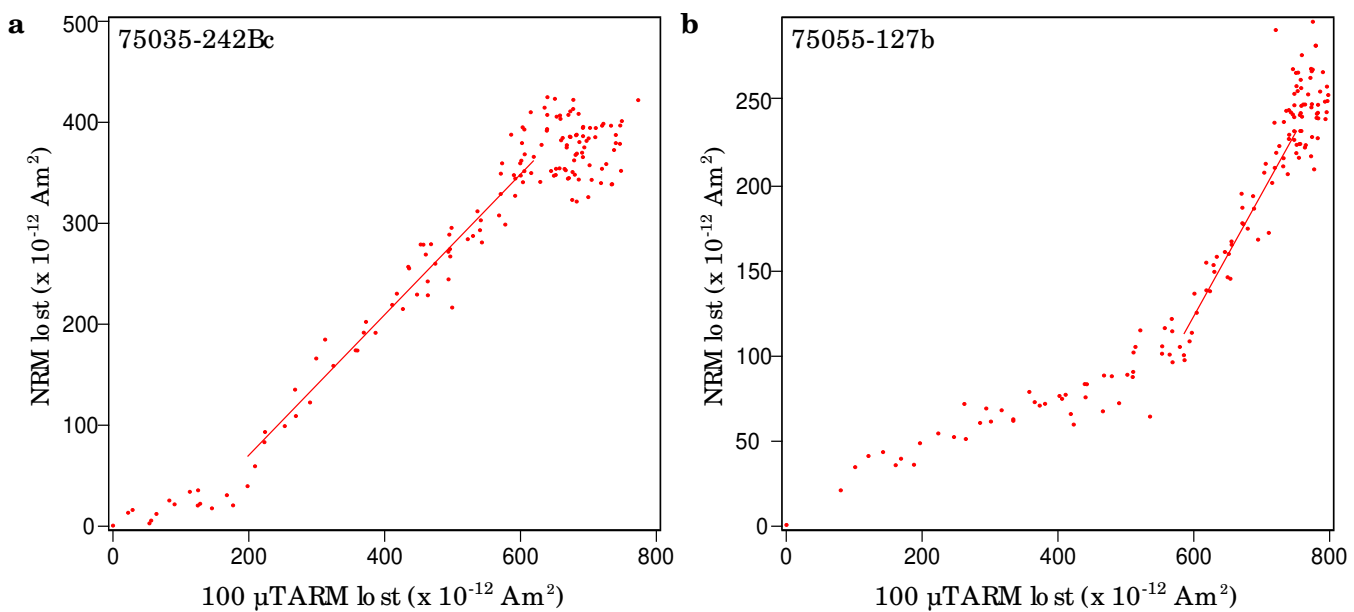

Figure S18 | Examples of ARM paleointensity estimates for 75035 and 75055. NRM lost versus $100 \mu \mathrm{T}$ ARM lost is plotted for progressive AF demagnetization. (a) ARM paleointensity plot for specimen 75035-242Bc. (b) ARM paleointensity plot for specimen 75055-127b.

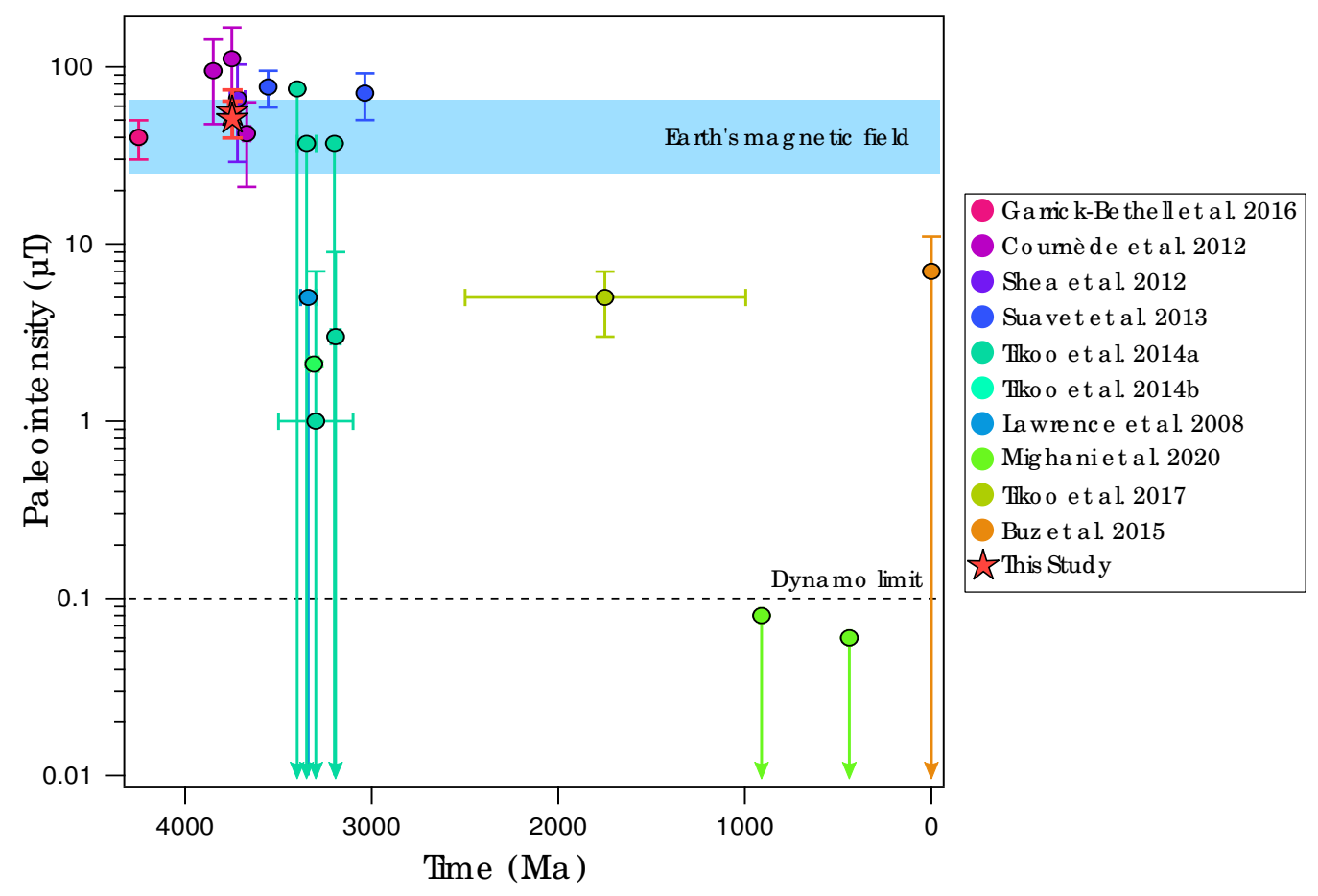

Figure S19 | Summary of the paleointensity measurements on Apollo samples colour coded by study from refs. 2, 3, 5, 26, 46, 47, 48, 65, 86, 96, 97. The blue region is the strength of Earth's present day magnetic field. The dashed black line is the weakest field that could be driven by a lunar dynamo (see ref. 3). Paleointensities calculated for 75035 and 75055 are shown by red stars. 

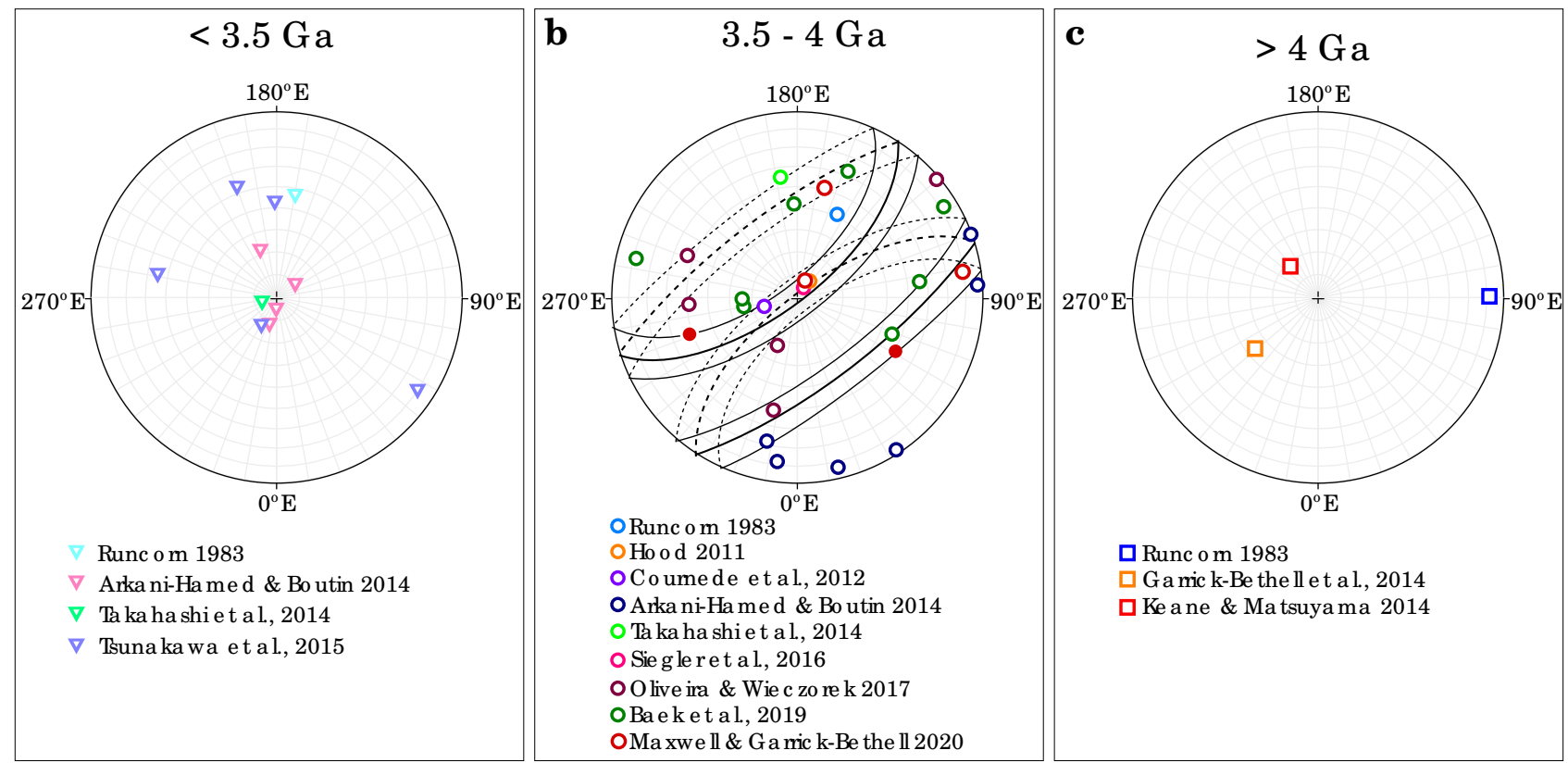

Figure S20 | A summary of north paleopole locations summarized from the literature. (a) Paleopole locations for $<3.5 \mathrm{Ga}$ are generally found to be aligned along the present day spin axis $^{51,55,98,99}$. (b) Paleopole locations $3.5-4 \mathrm{Ga}^{5,6,7,10,51,52,55,98,100}$ which have ages broadly similar to our samples (the permitted North pole locations from our paleoinclination are shown by the two small circles with 95\% confidence bounds). (c) Paleopole locations > $4 \mathrm{Ga}^{46,55,101}$. 

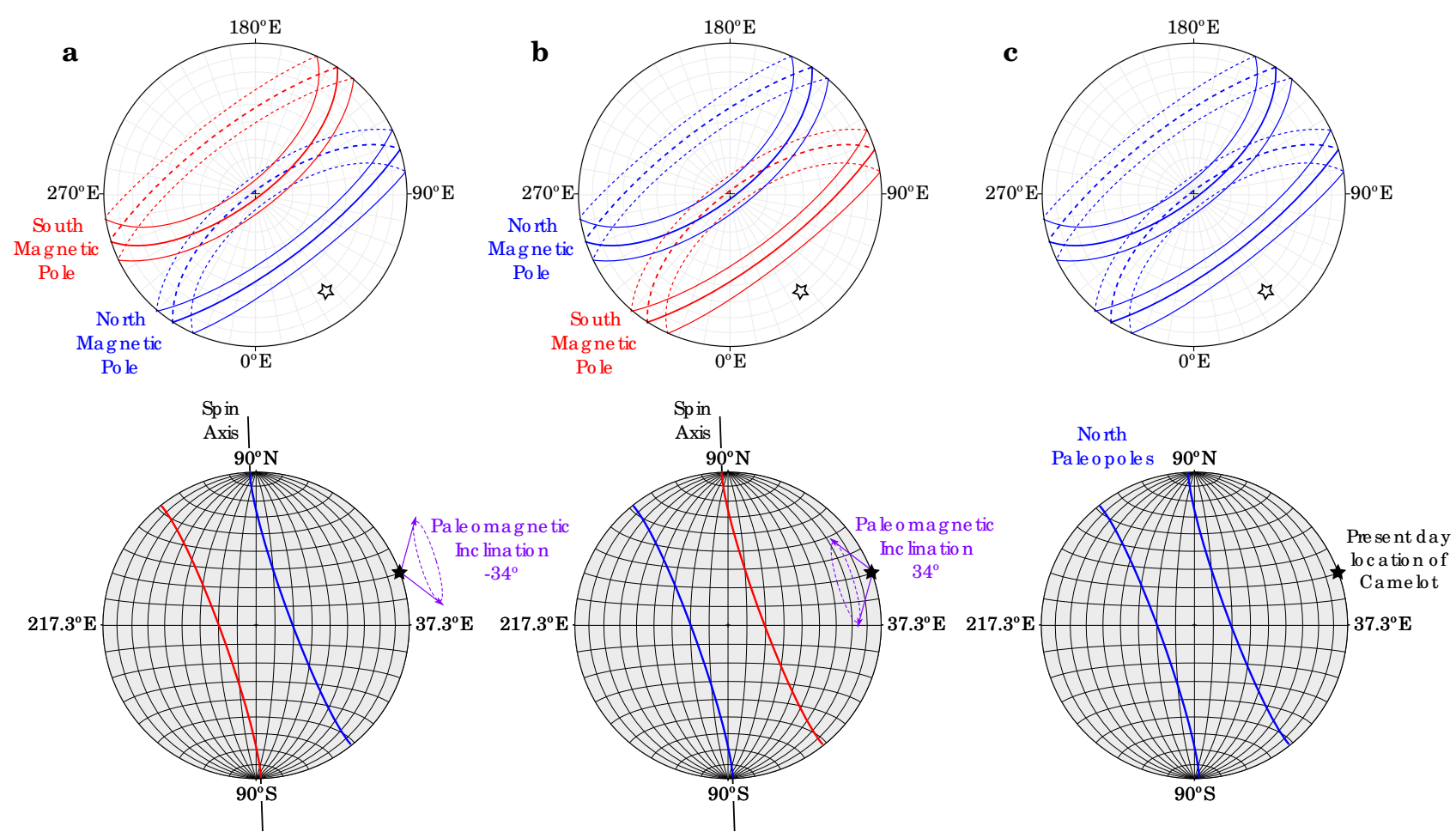

Figure S21 | Schematic diagrams show the plausible true polar wander paths permitted by our measured paleoinclination for 75055 . The blue and red small circles show the permitted locations of the north and south magnetic poles, respectively. We assume a selenocentric axial dipole such that the paleopole location represents the position of the ancient spin axis. (a) If the measured magnetic inclination at Camelot crater is negative, Camelot was in the northern hemisphere $3.7 \mathrm{Ga}$ ago and was located closest the north magnetic pole. (b) If the measured magnetic inclination at Camelot crater is positive, Camelot was in the southern hemisphere $3.7 \mathrm{Ga}$ ago and was located closest to the south magnetic pole. (c) Considering Camelot crater at its present day location, the small circles are shown for the allowed north pole locations for Camelot crater in either the northern or southern hemisphere $3.7 \mathrm{Ga}$ ago. 


\section{S3 Distinguishing between sources of the lunar magnetic field in light of new paleoinclination results}

Given the difficulties associated with generating the observed strong lunar magnetic field with a thermal or thermochemical core dynamo, a diversity of other dynamo as well as non-dynamo mechanisms have been proposed to have magnetized the lunar surface. We consider these mechanisms here for completeness, but acknowledge that they are less likely given our current understanding of the lunar interior.

\section{S3.1 Multipolar magnetic fields}

The high value of $R o_{l}$ for the lunar core resulting from its slow rotation rate suggests the dynamo should be multipolar, at least in the dynamo-generating region (main text Figure $1 \mathrm{~b}$ ) ${ }^{14}$. Alternatively, it has been suggested that rather than originating in the core, the lunar magnetic field may have been generated by a basal magma ocean dynamo (main text Figure 1c,d), which would be closer to the lunar surface which would favour a higher degree of multipolarity at the surface relative to that of a deeper-seated core dynamo. As such, the measured paleoinclination may not constrain the axial dipole field component. The inclination of the magnetic field at Camelot crater depends on the zonal dipolar, quadrupolar and octupolar contributions to the magnetic field ${ }^{8}$ as:

$$
\tan I=\frac{2 \cos \theta+G 2\left(4.5 \cos ^{2} \theta-1.5\right)+G 3\left(10 \cos ^{3} \theta-6 \cos \theta\right)}{\sin \theta+G 2(3 \cos \theta \sin \theta)+G 3\left(7.5 \cos ^{2} \theta \sin \theta-1.5 \sin \theta\right)}
$$

where $I$ is the inclination and $\theta$ is the colatitude which we take to be $69.8^{\circ}$ (the current colatitude of Camelot crater, assuming no true polar wander since remanence acquisition), $G 2$ is the zonal quadrupole to zonal dipole field ratio, and $G 3$ is the zonal octupole to zonal dipole field ratio. Both low $(G 2<0.2$ and $G 3<0.2)$ and high $(0<G 2<1$ and $0.3<G 3<0.75)$ contributions from the quadrupolar and octupolar components give results consistent with our measured inclinations. Therefore, even when considering just the first two nondipolar zonal terms to the multipole field expansion, the lunar dynamo is permitted to be highly multipolar based on our measured paleoinclination, consistent with either a dynamo originating in the core or a basal magma ocean. 


\section{S3.2 External magnetic field sources}

We consider whether the lunar surface could plausibly have been magnetized by Earth's magnetic field. We assume the Earth had an active magnetic field $\sim 3.7 \mathrm{Ga}$ ago, although there is currently no robust record beyond $3.5 \mathrm{Ga}$ ago $102,103,104,105$. Under the assumption that the mean field represented an axial dipole, and given that the Earth and Moon were likely already tidally locked by this time ${ }^{106}$, such a field would have uniformly magnetized the lunar surface, with $I=\lambda$ (main text Figure 1e). In this case, the inclination of the magnetic field at Camelot crater would be much lower than the observed paleoinclinations, confirming the Earth's magnetic field is an unlikely source of lunar magnetization. In addition, given the distance between the Earth and the Moon, the Earth would impart negligible paleointensities $(\sim 1 \mu \mathrm{T}$ assuming the Earth's magnetic field is similar to today, and the Moon was at the Roche limit) on the lunar surface.

We also consider the influence of the IMF and its ability to magnetize the lunar surface. The IMF constantly changes direction, but the time-averaged field is predominantly aligned in the lunar equatorial plane ${ }^{29}$, where $I=90-\lambda$ (main text Figure 1f). Assuming a similar geometry at $3.7 \mathrm{Ga}$, the IMF would therefore have generated a much higher inclination than that observed at Camelot crater, ruling out this source of magnetization. In addition, because the Moon rotates $360^{\circ}$ with respect to the IMF every orbit around the Earth, the mean field at Camelot should average out to a null field.

\section{S3.3 Impact-generated magnetic fields}

It has been suggested that the lunar surface may have been magnetized by impact events ${ }^{107}$. In particular, it was proposed that basin-forming impacts could generate a plasma field which expands around the Moon, focusing the magnetic field at the antipodal point to the impact ${ }^{31}$. Although recent magnetohydrodynamic simulations indicate that such a mechanism likely cannot explain the high paleointensities recorded by lunar samples ${ }^{30}$, we still compare its predictions with our measurements as an experimental test of this hypothesis. Independent of latitude, crustal magnetization generated by impact events would lead to near-vertical paleoinclinations (here we assume they are perfectly vertical) (main text Figure 1g,h). Our recovered paleoinclination at Camelot crater is $\sim 50^{\circ}$ shallower, suggesting this is an unlikely source of magnetization.

We also consider whether the magnetizations of 75035 and 75055 are compatible with having been acquired during the Camelot crater formation event at 500 Ma ago. Because the dynamo had likely 
ceased by this time ${ }^{3}$, the only plausible field source would be the local crustal field. We assume that the local crustal field was the same as that today and was not modified by the cratering event. We test this hypothesis in two ways: first, we compare the measured paleoinclination to that of the crustal field and second, we estimate whether the samples could have been sufficiently heated or shocked by the impact to acquire a stable NRM. The crustal magnetic field at an altitude of $30 \mathrm{~km}$ above the lunar surface has been measured by the Lunar Prospector fluxgate magnetometer ${ }^{108}$. Using both sequential and correlative field models we found that the inclination of the crustal magnetic field at Camelot crater is $\sim 47^{\circ}$ which differs by more than $2 \sigma$ from our measured paleoinclination from 75055 .

We now consider whether the samples would likely have been remagnetized by the impact. We conducted impact simulations using the iSALE-2D shock physics code ${ }^{109,110,111}$ to evaluate the potential for shock metamorphism and heating from the formation of Camelot crater. The impact simulations were run to match the dimensions of Camelot crater, with a final rim-to-rim diameter of $\sim 600 \mathrm{~m}$ and original depth of $\sim 120 \mathrm{~m}^{4}$. To achieve these dimensions, a $20 \mathrm{~m}$ diameter, spherical impactor travelling at $15 \mathrm{~km} \mathrm{~s}^{-1}$ (a typical lunar impact velocity ${ }^{112,113}$ ) perpendicular to the target was used. Both the impactor and target were assumed to have zero porosity and were simulated using an ANEOS equation of state for basalt ${ }^{114}$. Simulations were run with a spatial resolution of $2 \mathrm{~m}$.

Our simulations suggest low peak pressures $(<1 \mathrm{GPa})$ and post-shock temperatures $(<280 \mathrm{~K})$ associated with the Camelot crater-forming impact event at the inferred formation locations of 75035 and 75055. Results are shown for crater formation at the transient cavity stage (Figure S22). Only a small volume of material, proximal to the point of impact experiences significant shock pressures or post-shock temperature increases. However, attenuation would be much greater in a porous target (e.g., one or more thick layers of intra-basalt regolith layers) so an even smaller volume may experience temperatures $>100{ }^{\circ} \mathrm{C}$ greater than normal ${ }^{115}$. Temperatures are therefore unlikely to exceed the Curie temperature given the relatively low porosity of mare basalts ${ }^{116}$. The peak shock pressure is also too low to influence the NRM of the samples. Paleomagnetic signals preserved in samples prior to cratering at this locality are therefore likely to be unperturbed. 

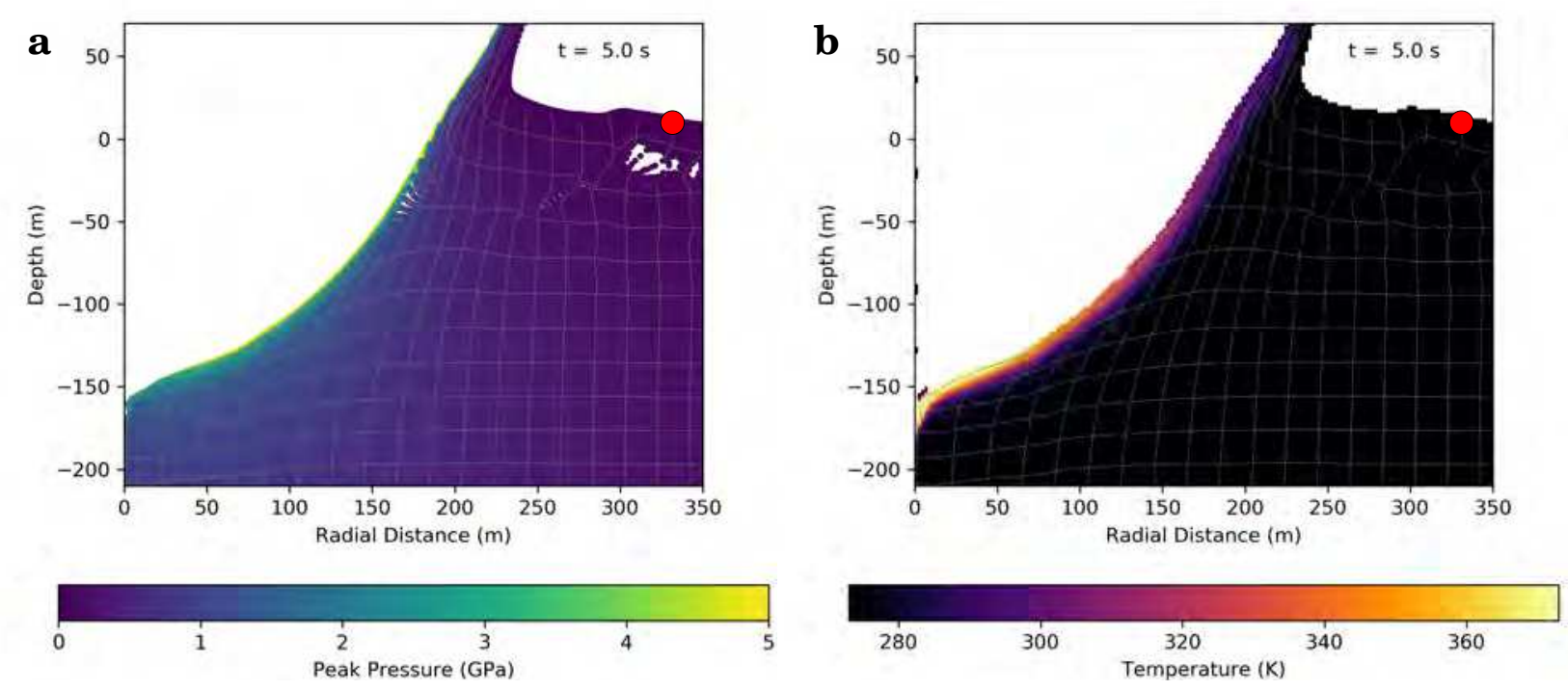

Figure S22 | Impact simulations for a $20 \mathrm{~m}$ diameter, spherical impactor with a velocity of $15 \mathrm{~km} \mathrm{~s}^{-1}$. Simulations show the peak pressure and temperature 5 seconds after the impact event. Both the target and impactor are basaltic in composition. The red dot indicates the approximate position of 75035 and 75055 at Station 5. (a) The bulk of the target material is only very weakly shocked, with peak pressures predominantly $<2 \mathrm{GPa}$. (b) Only the edge of the impact basin experiences significant heating, while the bulk of the target material does not exceed temperatures of $280 \mathrm{~K}$. 


\section{References}

[1] Weiss, B. P. \& Tikoo, S. M. The lunar dynamo. Science 346 (2014).

[2] Tikoo, S. M. et al. A two-billion-year history for the lunar dynamo. Science Advances 3, e1700207 (2017).

[3] Mighani, S. et al. The end of the lunar dynamo. Science Advances 6, 1-8 (2020).

[4] Schmitt, H. H. et al. Revisiting the field geology of Taurus-Littrow. Icarus 298, 2-33 (2017).

[5] Cournède, C., Gattacceca, J. \& Rochette, P. Magnetic study of large Apollo samples: Possible evidence for an ancient centered dipolar field on the Moon. Earth and Planetary Science Letters 331-332, 31-42 (2012).

[6] Hood, L. L. Central magnetic anomalies of Nectarian-aged lunar impact basins: Probable evidence for an early core dynamo. Icarus 211, 1109-1128 (2011).

[7] Siegler, M. A. et al. Lunar true polar wander inferred from polar hydrogen. Nature 531, 480-484 (2016)

[8] Merrill, R. T., McElhinny, M. \& McFadden, P. L. The magnetic field of the Earth, vol. 27 (1996).

[9] Potter, D. K. Novel magnetic techniques for rapidly detecting palaeomagnetically important single-domain iron particles and obtaining directional palaeomagnetic data from "unoriented" lunar rock samples. Canadian Aeronautical Space Journal 57, 12-23 (2011).

[10] Oliveira, J. S. \& Wieczorek, M. A. Testing the axial dipole hypothesis for the Moon by modeling the direction of crustal magnetization. Journal of Geophysical Research 122, 383-399 (2017).

[11] Vervelidou, F. \& Lesur, V. Unveiling Earth's hidden magnetization. Geophysical Research Letters 45, 12,283-12,292 (2018).

[12] Gerhards, C. On the reconstruction of inducing dipole directions and susceptibilities from knowledge of the magnetic field on a sphere. Inverse Problems in Science and Engineering 27, 37-60 (2019).

[13] Gastine, T., Duarte, L. \& Wicht, J. Dipolar versus multipolar dynamos: The influence of the background density stratification. Astronomy and Astrophysics 546, 1-13 (2012).

[14] Olson, P. \& Christensen, U. R. Dipole moment scaling for convection-driven planetary dynamos. Earth and Planetary Science Letters 250, 561-571 (2006).

[15] Stevenson, D. J. Planetary magnetic fields: Achievements and prospects. Space Science Reviews 152, 651-664 (2009).

[16] Weber, R. C., Lin, P.-Y., Garnero, E., Williams, Q. C. \& Lognonné, P. Seismic detection of the lunar core. Science 331, 309-12 (2011).

[17] Cao, H. et al. A dynamo explanation for Mercury's anomalous magnetic field. Geophysical Research Letters 41, 4127-4134 (2014).

[18] Christensen, U. R. A deep dynamo generating Mercury's magnetic field. Nature 444, 1056-1058 (2006) 
[19] Takahashi, F., Shimizu, H. \& Tsunakawa, H. Mercury's anomalous magnetic field caused by a symmetry-breaking self-regulating dynamo. Nature Communications 10, 1-8 (2019).

[20] Manglik, A., Wicht, J. \& Christensen, U. R. A dynamo model with double diffusive convection for Mercury's core. Earth and Planetary Science Letters 289, 619-628 (2010).

[21] Dwyer, C. A., Stevenson, D. J. \& Nimmo, F. A long-lived lunar dynamo driven by continuous mechanical stirring. Nature 479, 212-214 (2011).

[22] Stys, C. \& Dumberry, M. A past lunar dynamo thermally driven by the precession of its inner core. Journal of Geophysical Research 1-20 (2020).

[23] Evans, A. J., Tikoo, S. M. \& Andrews-Hanna, J. C. The case against an early lunar dynamo powered by core convection. Geophysical Research Letters 98-107 (2018).

[24] Cébron, D., Laguerre, R., Noir, J. \& Schaeffer, N. Precessing spherical shells: flows, dissipation, dynamo and the lunar core. Geophysical Journal International 219, S34-S57 (2019).

[25] Le Bars, M., Wieczorek, M. A., Karatekin, Ö., Cébron, D. \& Laneuville, M. An impact-driven dynamo for the early Moon. Nature 479, 215-218 (2011).

[26] Suavet, C. et al. Persistence and origin of the lunar core dynamo. Proceedings of the National Academy of Sciences 110, 8453-8 (2013).

[27] Scheinberg, A. L., Soderlund, K. M. \& Elkins-Tanton, L. T. A basal magma ocean dynamo to explain the early lunar magnetic field. Earth and Planetary Science Letters 492, 144-151 (2018).

[28] Daily, W. \& Dyal, P. Theories for the Origin of Lunar Magnetism. Physics of the Earth and Planetary Interiors 20, 255-270 (1979).

[29] Oran, R., Weiss, B. P. \& Cohen, O. Were chondrites magnetized by the early solar wind? Earth and Planetary Science Letters 492, 222-231 (2018).

[30] Oran, R., Weiss, B. P., Shprits, Y., Miljković, K. \& Tóth, G. Was the moon magnetized by impact plasmas? Science Advances 6, 1-11 (2020).

[31] Hood, L. L. Magnetic field and remanent magnetization effects of basin-forming impacts on the Moon. Geophysical Research Letters 14, 844-847 (1987).

[32] Hide, R. Comments on the moon's magnetism. The Moon 4 (1972).

[33] Srnka, L. J. et al. Magnetic field and shock effects and remanent magnetization in a hypervelocity impact experiment. Earth and Planetary Science Letters 42, 127-137 (1979).

[34] Crawford, D. A. \& Schultz, P. H. The production and evolution of impact-generated magnetic fields. International Journal of Impact Engineering 14, 205-216 (1993).

[35] Crawford, D. A. Simulations of magnetic fields produced by asteroid impact: Possible implications for planetary paleomagnetism. International Journal of Impact Engineering 137, 1-8 (2020).

[36] Dyal, P., Parkin, C. W. \& Daily, W. D. Global lunar crust: Electrical conductivity and thermoelectric origin of remanent magnetism. 8th Lunar Science Conference 767-783 (1977). 
[37] Gold, T. \& Soter, S. Cometary impact and the magnetization of the Moon. Planetary and Space Science 24, 45-54 (1976).

[38] Schultz, P. H. \& Srnka, L. J. Cometary collisions on the Moon and Mercury. Nature 284, 22-26 (1980).

[39] Snape, J. F. et al. The timing of basaltic volcanism at the Apollo landing sites. Geochimica et Cosmochimica Acta 266, 29-53 (2019). URL https://doi.org/10.1016/j.gca.2019.07.042.

[40] Davies, M. E. \& Colvin, T. R. Lunar coordinates in the regions of the Apollo landers. Journal of Geophysical Research 105, 20277-20280 (2000).

[41] Apollo Lunar Geology Investigation Team. Geologic Setting of the Apollo 15 Samples. Science 175, 407-415 (1972).

[42] Donohue, P. H. \& Neal, C. R. Quantitative textural analysis of ilmenite in Apollo 17 hightitanium mare basalts. Geochimica et Cosmochimica Acta 149, 115-130 (2015).

[43] Usselman, T. M., Lofgren, G. E., Williams, R. J. \& Donaldson, C. H. Experimentally reproduced textures and mineral chemistries of high-titanium mare basalts. Lunar and Planetary Science Conference Proceedings 6, 997-1020 (1975).

[44] Ahrens, T. J., O'Keefe, J. D. \& Gibbons, R. V. Shock compression of a recrystallized anorthositic rock from Apollo 15. Proceedings of the Fourth Lunar Science Conference 3, 2575-2590 (1973).

[45] Schaal, R. B. \& Hörz, F. Shock metamorphism of lunar and terrestrial basalts. Proceedings of the Lunar Science Conference 8, 1697-1729 (1977).

[46] Garrick-Bethell, I., Perera, V., Nimmo, F. \& Zuber, M. T. The tidal-rotational shape of the Moon and evidence for polar wander. Nature 512, 181-184 (2014).

[47] Shea, E. K. et al. A long-lived lunar core dynamo. Science 335, 453-456 (2012).

[48] Tikoo, S. M. et al. Decline of the lunar core dynamo. Earth and Planetary Science Letters 404, 89-97 (2014).

[49] Schubert, G. \& Soderlund, K. M. Planetary magnetic fields: Observations and models. Physics of the Earth and Planetary Interiors 187, 92-108 (2011).

[50] Russell, C. T. \& Dougherty, M. K. Magnetic fields of the outer planets. Space Science Reviews 152, 251-269 (2010).

[51] Takahashi, F., Tsunakawa, H., Shimizu, H., Shibuya, H. \& Matsushima, M. Reorientation of the early lunar pole. Nature Geoscience 7, 409-412 (2014).

[52] Maxwell, R. E. \& Garrick-Bethell, I. Evidence for an ancient near-equatorial lunar dipole from higher precision inversions of crustal magnetization. Journal of Geophysical Research 125, 1-20 (2020).

[53] Nayak, M., Hemingway, D. \& Garrick-Bethell, I. Magnetization in the South Pole-Aitken basin: Implications for the lunar dynamo and true polar wander. Icarus 286, 153-192 (2017).

[54] Hood, L. L. \& Artemieva, N. A. Antipodal effects of lunar basin-forming impacts: Initial 3D simulations and comparisons with observations. Icarus 193, 485-502 (2008). 
[55] Runcorn, S. K. Lunar magnetism, polar displacements and primeval satellites in the Earth-Moon system. Nature 304, 589-596 (1983).

[56] Wolfe, E. W. et al. Investigation of the Taurus-Littrow Valley : Apollo 17 Landing Site The Geologic. Geological Survey Professional Paper 1080 (1981).

[57] Meyer, C. Lunar Sample Compendium. Lunar and Planetary Science Conference 1016 (2010).

[58] Cashman, K. V., Thornber, C. R. \& Kauahikaua, J. P. Cooling and crystallization of lava in open channels, and the transition of pahoehoe lava to 'a'a. Bulletin of Volcanology 61, 306-323 (1999).

[59] Self, S., Keszthelyi, L. \& Thordarson, T. The importance of pahoehoe. Annu. Rev. Earth Planet. Sci 26, 81-110 (1998).

[60] Brown, G. M., Peckett, A., Emeleus, C. H., Phillips, R. \& Pinsent, R. H. Petrology and mineralogy of Apollo 17 mare basalts. Proceedings of the Lunar Science Conference 6, 1-13 (1975).

[61] Murase, T. \& Mcbirney, A. R. Viscosity of lunar lavas. Science 167, 1491-1493 (1970).

[62] Head, J. W. Lunar volcanism in space and time. Reviews of Geophysics 14, 265-300 (1976).

[63] Manga, M. Waves of bubbles in basaltic magmas and lavas. Journal of Geophysical Research 101, 17457-17465 (1996).

[64] Wang, H. et al. Lifetime of the solar nebula constrained by meteorite paleomagnetism. Science 627, 623-627 (2017).

[65] Tikoo, S. M. et al. Magnetic fidelity of lunar samples and implications for an ancient core dynamo. Earth and Planetary Science Letters 337-338, 93-103 (2012).

[66] Suavet, C., Weiss, B. P. \& Grove, T. L. Controlled-atmosphere thermal demagnetization and paleointensity analyses of extraterrestrial rocks. Geochemistry, Geophysics, Geosystems 15, 27332743 (2014).

[67] Garrick-Bethell, I., Weiss, B. P., Shuster, D. L. \& Buz, J. Early lunar magnetism. Science 323, 356-9 (2009).

[68] Kirschvink, J. L. The least-squares line and plane and the analysis of palaeomagnetic data. Geophysical Journal of the Royal Astronomical Society 62, 699-718 (1980).

[69] Tauxe, L. \& Staudigel, H. Strength of the geomagnetic field in the cretaceous normal superchron: New data from submarine basaltic glass of the troodos ophiolite. Geochemistry, Geophysics, Geosystems 5, 1-16 (2004).

[70] Tauxe, L. et al. PmagPy: Software package for paleomagnetic data analysis and a bridge to the Magnetics Information Consortium (MagIC) Database. Geochemistry, Geophysics, Geosystems 17, 2450-2463 (2016).

[71] Allmendinger, R. W., Cardozo, N. C. \& Fisher, D. Vectors \& Tensors. In Structural Geology Algorithms, 44-98 (Cambridge University Press, 2013).

[72] Cardozo, N. C. \& Allmendinger, R. W. Spherical projections with OSXStereonet. Computers \& Geosciences 51, 193-205 (2013). 
[73] Paterson, G. A., Heslop, D. \& Pan, Y. The pseudo-Thellier palaeointensity method: New calibration and uncertainty estimates. Geophysical Journal International 207, 1596-1608 (2016).

[74] Stephenson, A. \& Collinson, D. W. Lunar magnetic field palaeointensities determined by an anhysteretic remanent magnetization method. Earth and Planetary Science Letters 23, 220-228 (1974).

[75] Tauxe, L. Essentials of Paleomagnetism (University of California Press, 2010).

[76] Halgedahl, S. L., Day, R. \& Fuller, M. The effect of cooling rate on the intensity of weak-field TRM in single-domain magnetite. Journal of Geophysical Research 85, 3690-3698 (1980).

[77] Bryson, J. F., Weiss, B. P., Harrison, R. J., Herrero-Albillos, J. \& Kronast, F. Paleomagnetic evidence for dynamo activity driven by inward crystallisation of a metallic asteroid. Earth and Planetary Science Letters 472, 152-163 (2017).

[78] Press, W., Teukolsky, S., Vertterling, W. \& Flannery, B. Savitzky-Golay Smoothing Filters. In Numerial Recipes in C, The Art of Scientific Computing, 650-655 (Cambridge University Press, 1992), 2nd edn.

[79] Jelinek, V. Characterization of the magnetic fabric of rocks. Tectonophysics 79, 63-67 (1981).

[80] Girdler, R. W. The measurement and computation of anisotropy of magnetic susceptibility of rocks. Geophysical Journal of the Royal Astronomical Society 5, 34-44 (1961).

[81] Paces, J. B. et al. A strontium and neodymium isotopic study of Apollo 17 high-Ti mare basalts: Resolution of ages, evolution of magmas, and origins of source heterogeneities. Geochimica et Cosmochimica Acta 55, 2025-2043 (1991).

[82] Turner, G. \& Cadogan, P. H. Possible effects of 39Ar recoil in 40Ar-39Ar dating. Proceedings of the Fifth Lunar Conference 2, 1601-1615 (1974).

[83] Turner, G., Cadogan, P. H. \& Yonge, C. J. Argon selenochronology. Proceedings of the Fourth Lunar Science Conference 2, 1889-1914 (1973).

[84] Pearce, G. Magnetism of the Apollo 17 samples. Lunar and Planetary Science Conference 5, 590 (1974).

[85] Sugiura, N., Strangway, D. \& Pearce, G. Heating experiments and paleointensity determinations. Proceedings of the Lunar and Planetary Science Conference 9, 3151-3163 (1978).

[86] Lawrence, K., Johnson, C., Tauxe, L. \& Gee, J. Lunar paleointensity measurements: Implications for lunar magnetic evolution. Physics of the Earth and Planetary Interiors 168, 71-87 (2008).

[87] Archanjo, C. J. \& Launeau, P. Magma flow inferred from preferred orientations of plagioclase of the Rio Cearfi-Mirim dyke swarm (NE Brazil) and its AMS significance. Geological Society Special Publication 238, 285-298 (2004).

[88] Geoffroy, L., Callot, J. P., Aubourg, C. \& Moreira, M. Magnetic and plagioclase linear fabric discrepancy in dykes: A new way to define the flow vector using magnetic foliation. Terra Nova 14, 183-190 (2002).

[89] Schindelin, J. et al. Fiji: an open-source platform for biological-image analysis. Nature Methods 9, 676-82 (2012). 
[90] Schindelin, J., Rueden, C. T., Hiner, M. C. \& Eliceiri, K. W. The ImageJ ecosystem: An open platform for biomedical image analysis. Molecular Reproduction and Development 82, 518-529 (2015).

[91] Muxworthy, A. R. \& Williams, W. Critical single-domain grain sizes in elongated iron particles: Implications for meteoritic and lunar magnetism. Geophysical Journal International 202, 578 $583(2015)$.

[92] Schobel, S. \& de Wall, H. AMS-NRM interferences in the Deccan basalts: Toward an improved understanding of magnetic fabrics in flood basalts. Journal of Geophysical Research 119, 26512678 (2014).

[93] Whitford-Stark, J. L. Factors influencing the morphology of volcanic landforms: An earth-moon comparison. Earth Science Reviews 18, 109-168 (1982).

[94] Biedermann, A. R., Jackson, M., Stillinger, M. D., Bilardello, D. \& Feinberg, J. M. Anisotropy of full and partial anhysteretic remanence across different rock types: 1-Are partial anhysteretic remanence anisotropy tensors additive? Tectonics 39, 1-16 (2020).

[95] Biedermann, A. R., Jackson, M., Bilardello, D. \& Feinberg, J. M. Anisotropy of full and partial anhysteretic remanence across different rock types: 2- Coercivity dependence of remanence anisotropy. Tectonics 39, 1-19 (2020).

[96] Tikoo, S. M., Weiss, B. P., Shuster, D. L. \& Fuller, M. D. How long did the lunar core dynamo persist? Lunar and Planetary Science Conference 45, 1-2 (2014).

[97] Buz, J., Weiss, B. P., Tikoo, S. M., Shuster, D. L. \& Gattacceca, J. Magnetism of a very young lunar glass. Journal of Geophysical Research: Planets (2015).

[98] Arkani-Hamed, J. \& Boutin, D. Analysis of isolated magnetic anomalies and magnetic signatures of impact craters: Evidence for a core dynamo in the early history of the Moon. Icarus 237, $262-277$ (2014).

[99] Tsunakawa, H., Takahashi, F., Shimizu, H., Shibuya, H. \& Matsushima, M. Surface vector mapping of magnetic anomalies over the Moon using Kaguya and Lunar Prospector observations. Journal of Geophysical Research 120, 1160-1185 (2015).

[100] Baek, S. M., Kim, K. H., Garrick-Bethell, I. \& Jin, H. Magnetic anomalies within the Crisium Basin: magnetization directions, source depths, and ages. Journal of Geophysical Research 124, 223-242 (2019).

[101] Keane, J. T. \& Matsuyama, I. Evidence for lunar true polar wander and a past low-eccentricity, synchronous lunar orbit. Geophysical Research Letters 41, 6610-6619 (2014).

[102] Biggin, A. J. et al. Palaeomagnetism of Archaean rocks of the Onverwacht Group, Barberton Greenstone Belt (southern Africa): Evidence for a stable and potentially reversing geomagnetic field at ca. 3.5Ga. Earth and Planetary Science Letters 302, 314-328 (2011).

[103] Usui, Y., Tarduno, J. A., Watkeys, M., Hofmann, A. \& Cottrell, R. D. Evidence for a 3.45billion-year-old magnetic remanence: Hints of an ancient geodynamo from conglomerates of South Africa. Geochemistry, Geophysics, Geosystems 10, 1-16 (2009).

[104] Weiss, B. P. et al. Secondary magnetic inclusions in detrital zircons from the Jack Hills, Western Australia and implications for the origin of the geodynamo microscopy. Geology 46 (2018). 
[105] Tang, F. et al. Secondary magnetite in ancient zircon precludes analysis of a Hadean geodynamo. Proceedings of the National Academy of Sciences 116, 407-412 (2019).

[106] Ward, W. Past orientation of the lunar spin axis. Science 189, 377-380 (1975).

[107] Lin, R. P., Anderson, K. A. \& Hood, L. L. Lunar surface magnetic field concentrations antipodal to young large impact basins. Icarus $\mathbf{7 4}, 529-541$ (1988).

[108] Purucker, M. E. \& Nicholas, J. B. Global spherical harmonic models of the internal magnetic field of the Moon based on sequential and coestimation approaches. Journal of Geophysical Research 115, 1-14 (2010).

[109] Amsden, A. A., Ruppel, H. M. \& Hirt, C. W. SALE : A Simplified ALE Computer Program for Fluid Flow at All Speeds. Los Alamos National Laboratories Report 101 (1980).

[110] Collins, G. S., Melosh, H. J. \& Ivanov, B. A. Modeling damage and deformation in impact simulations. Meteoritics and Planetary Science 231, 217-231 (2004).

[111] Wünnemann, K., Collins, G. S. \& Melosh, H. J. A strain-based porosity model for use in hydrocode simulations of impacts and implications for transient crater growth in porous targets. Icarus 180, 514-527 (2006).

[112] Johnson, B. C. et al. The formation of lunar multi-ring basins. Science 354, 441-444 (2016).

[113] Le Feuvre, M. \& Wieczorek, M. A. Nonuniform cratering of the Moon and a revised crater chronology of the inner Solar System. Icarus 214, 1-20 (2011).

[114] Pierazzo, E., Artemieva, N. A. \& Ivanov, B. Starting Conditions for Hydrothermal Systems Underneath Martian Craters: Hydrocode Modeling. Geological Society of America Special Paper 384, 443-457 (2005).

[115] Wünnemann, K., Collins, G. S. \& Osinski, G. R. Numerical modelling of impact melt production in porous rocks. Earth and Planetary Science Letters 269, 530-539 (2008).

[116] Kiefer, W. S., MacKe, R. J., Britt, D. T., Irving, A. J. \& Consolmagno, G. J. The density and porosity of lunar rocks. Geophysical Research Letters 39, 1-5 (2012). 
Figures
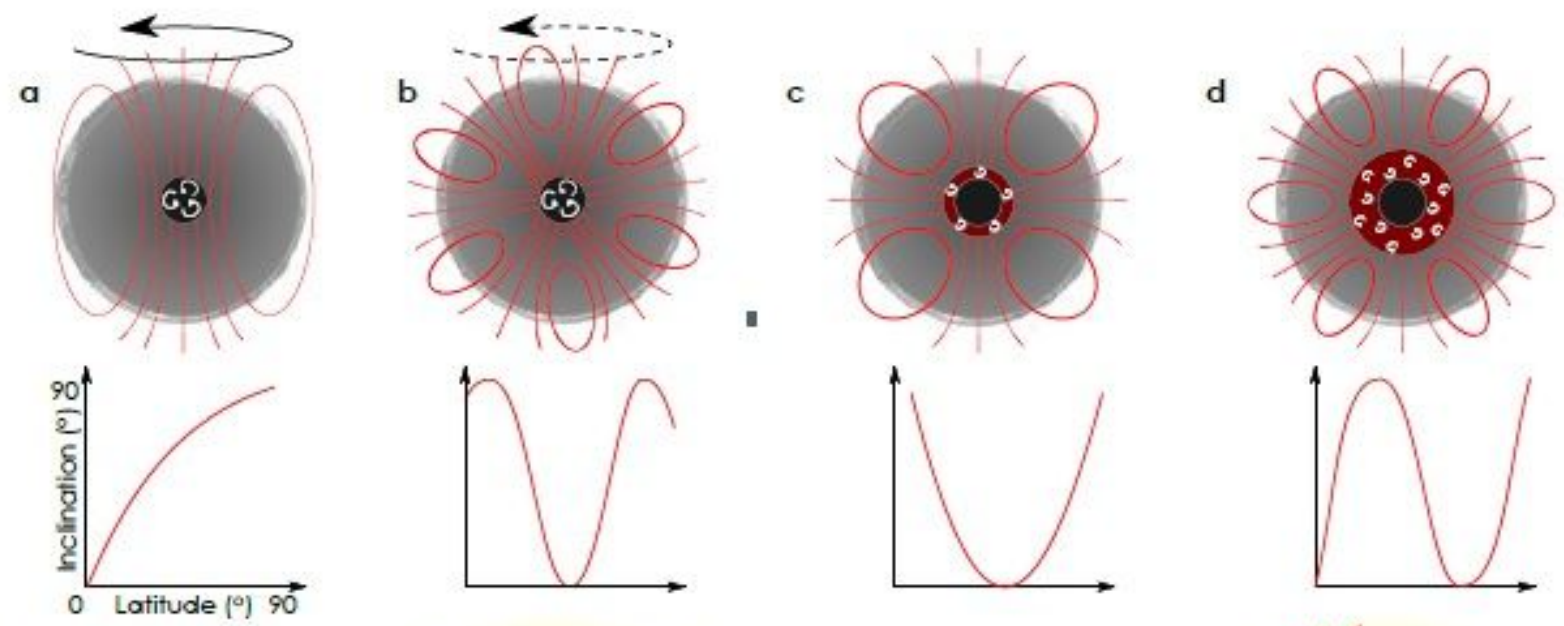

e
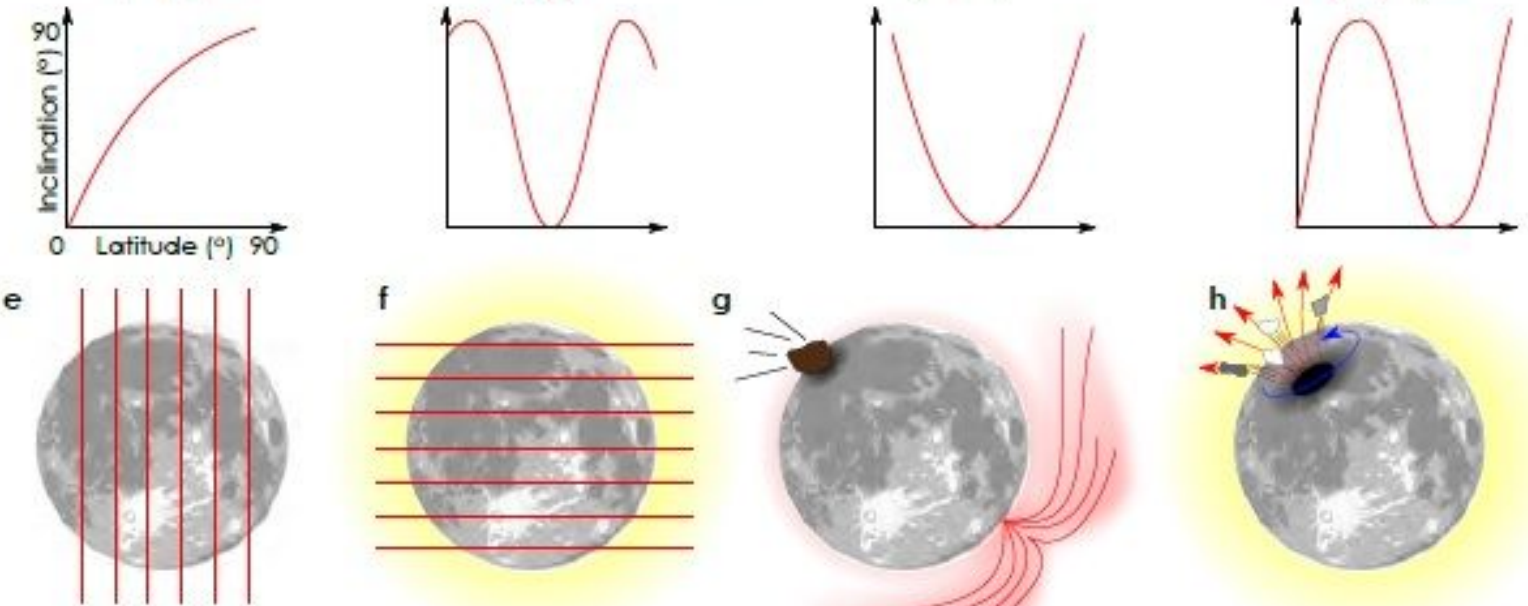

g
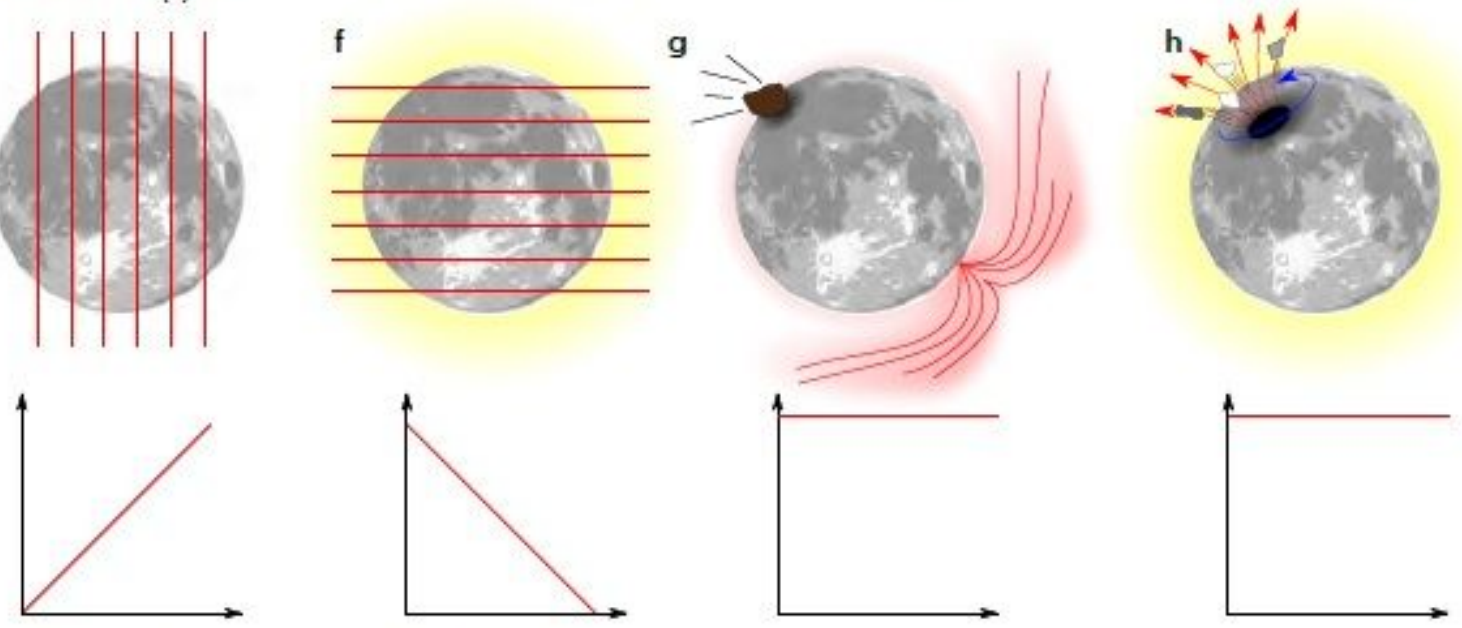

i
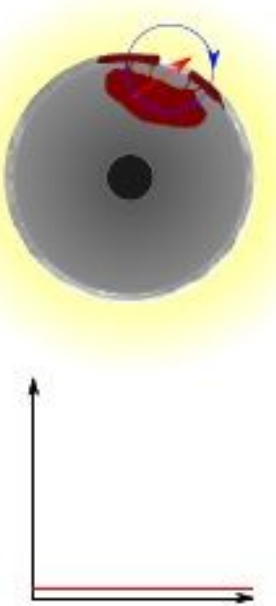

j
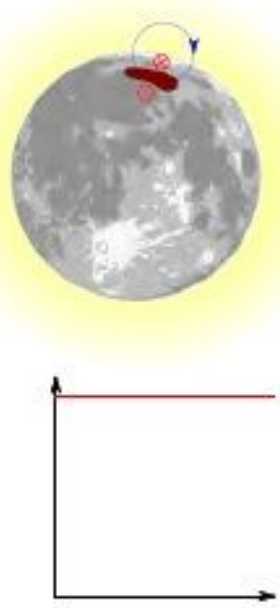

k
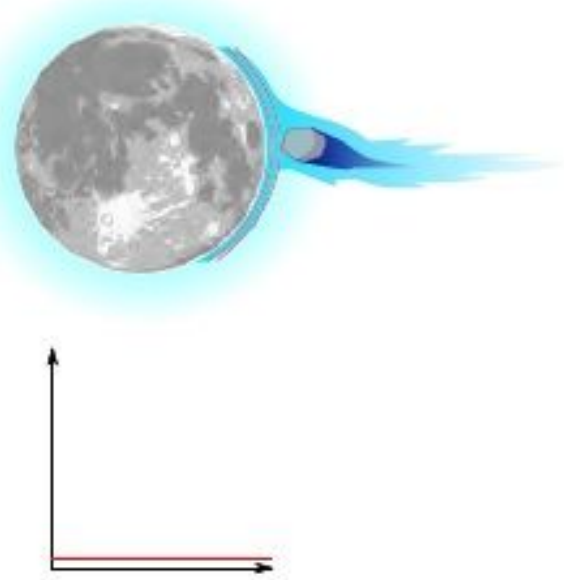

\section{Figure 1}

Predictions of various hypothesized lunar magnetic field sources for the paleoinclination of the lunar field. The top and bottom of each panel show a schematic of the approximate field direction with respect to the Moon and the inclination as a function of latitude, respectively. The black region represents the 
lunar core and dark red regions represent molten silicate. In all cases, red lines depict the magnetic field, blue lines depict any currents generated, and the yellow region around the Moon represents the solar wind when this is specifically required to generate the proposed magnetic field. a, A selenocentric axial dipolar magnetic field generated by a core dynamo with a rapid planetary rotation rate $(\mathrm{Rol}<0.12)$. b, A multipolar, non-axisymmetric magnetic field generated by a core dynamo with a slow planetary rotation rate (Rol > 0.12). c, An axially-aligned quadrupolar dynamo generated by a deep-sourced magma ocean dynamo27. d, An axially-aligned octupolar dynamo generated by a shallow-sourced magma ocean dynamo27. e, The Earth's mean axial dipole field at the location of the Moon. $f$, The IMF. $g$, Antipodal field amplified by basin forming impacts 54 . h, Fields generated by impact plasmas 28 . The predicted inclination is for rocks directly below the crater. i, Magnetic fields generated by the thermoelectric effect in lava basins that are electrically connected to subsurface magma and the solar wind28,36. j, Magnetic fields generated by a unipolar dynamo in lava basins 28 . The predicted inclination is for a rock at the surface close to the lava pool. $\mathrm{k}$, Compression of the IMF around the Moon by a cometary coma28.
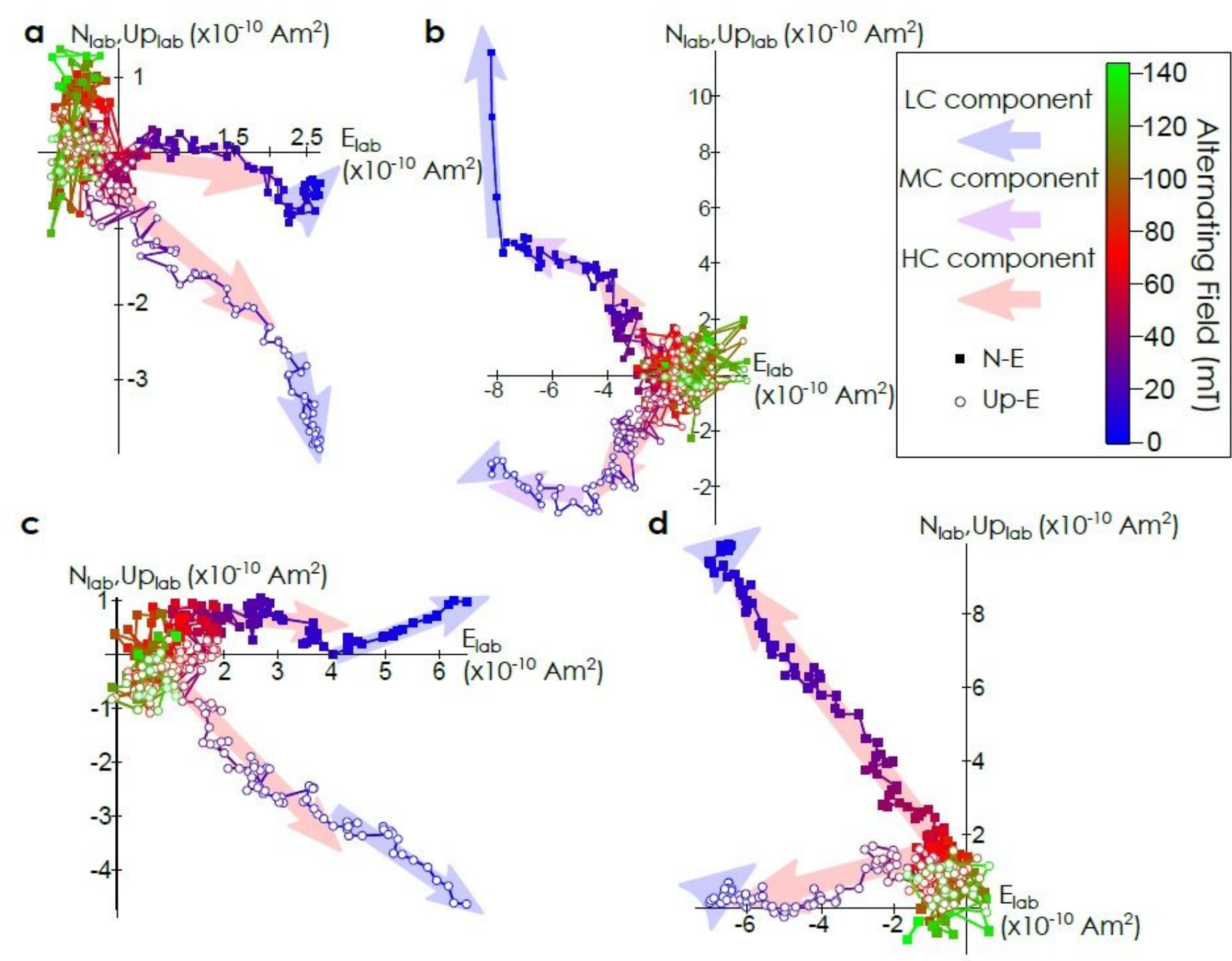

\section{Figure 2}


Demagnetization of NRM in 75035 and 75055. Orthographic projections in laboratory coordinates show the NRM vector during AF demagnetization projected along the North-East (closed squares) and Up-East (open symbols) directions. Stable components are shown by blue (LC), purple (medium coercivity, MC) and red $(\mathrm{HC})$ arrows, respectively. Data shown here are not corrected for remanence anisotropy. The peak AF is shown by the colour bar. a, Specimen 75035-242Ae. b, Specimen 75055-127Aa. c, Specimen 75035242Bg. d, Specimen 75055- 127Ae.
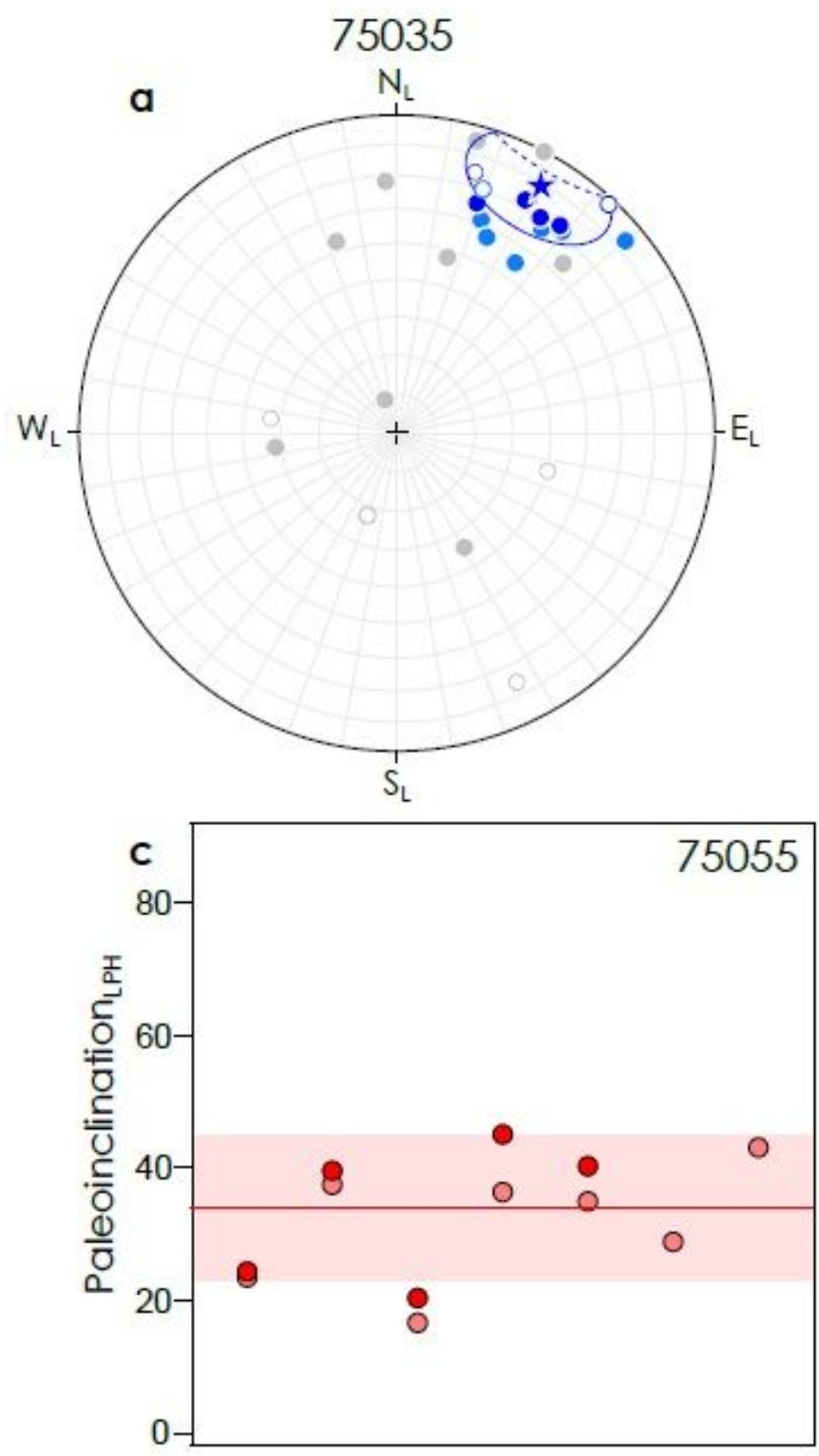

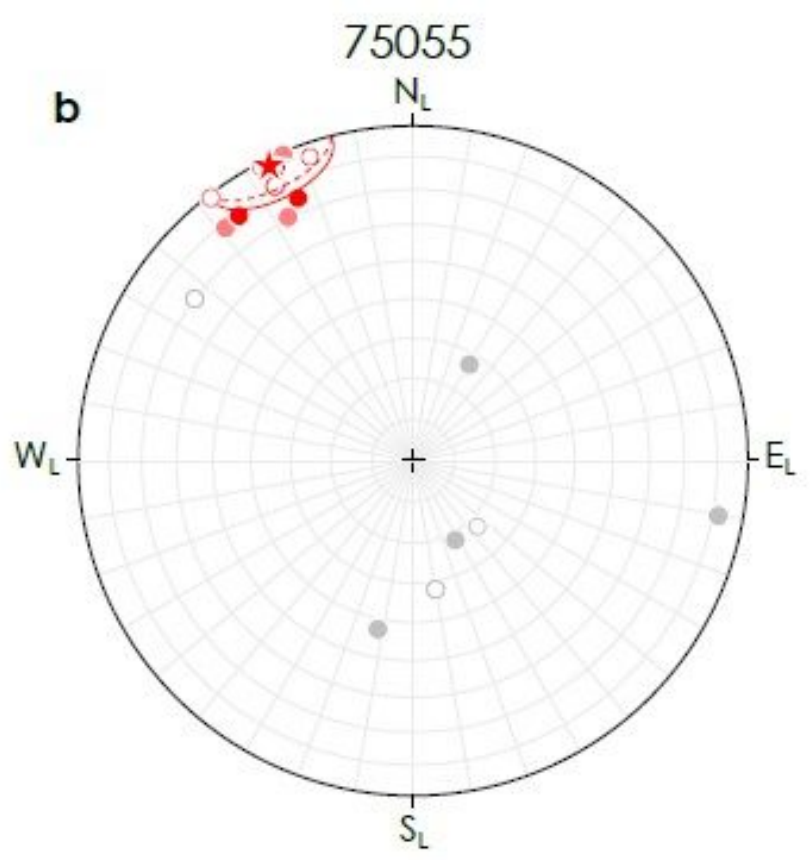

- Lower hemisphere

Upper hemisphere

LC component

-) HC component

-2 HC component, anisotropy-corrected

$\star \star$ Mean HC component

\section{Figure 3}

NRM components in 75035 and 75055 and associated paleoinclinations. The LC (grey) and HC (pale blue for 75035, pale red for 75055) components are shown in present-day lunar coordinates for individual specimens. Remanence-anisotropy-corrected HC components are shown in bright blue and red for 75035 and 75055 , respectively. The mean (stars) and 95\% confidence interval (ellipses) were calculated for the anisotropy-corrected $\mathrm{HC}$ components. a, Equal area stereonet projections of mutually oriented specimen 
components for 75035 in lunar coordinates. b, Equal area stereonet projections of mutually oriented specimen components for 75055 in lunar coordinates. c, Paleoinclinations in lunar paleohorizontal coordinates for the HC components in 75055. The mean (horizontal red line) and one standard deviation (red shaded region) are shown for anisotropy-corrected components.
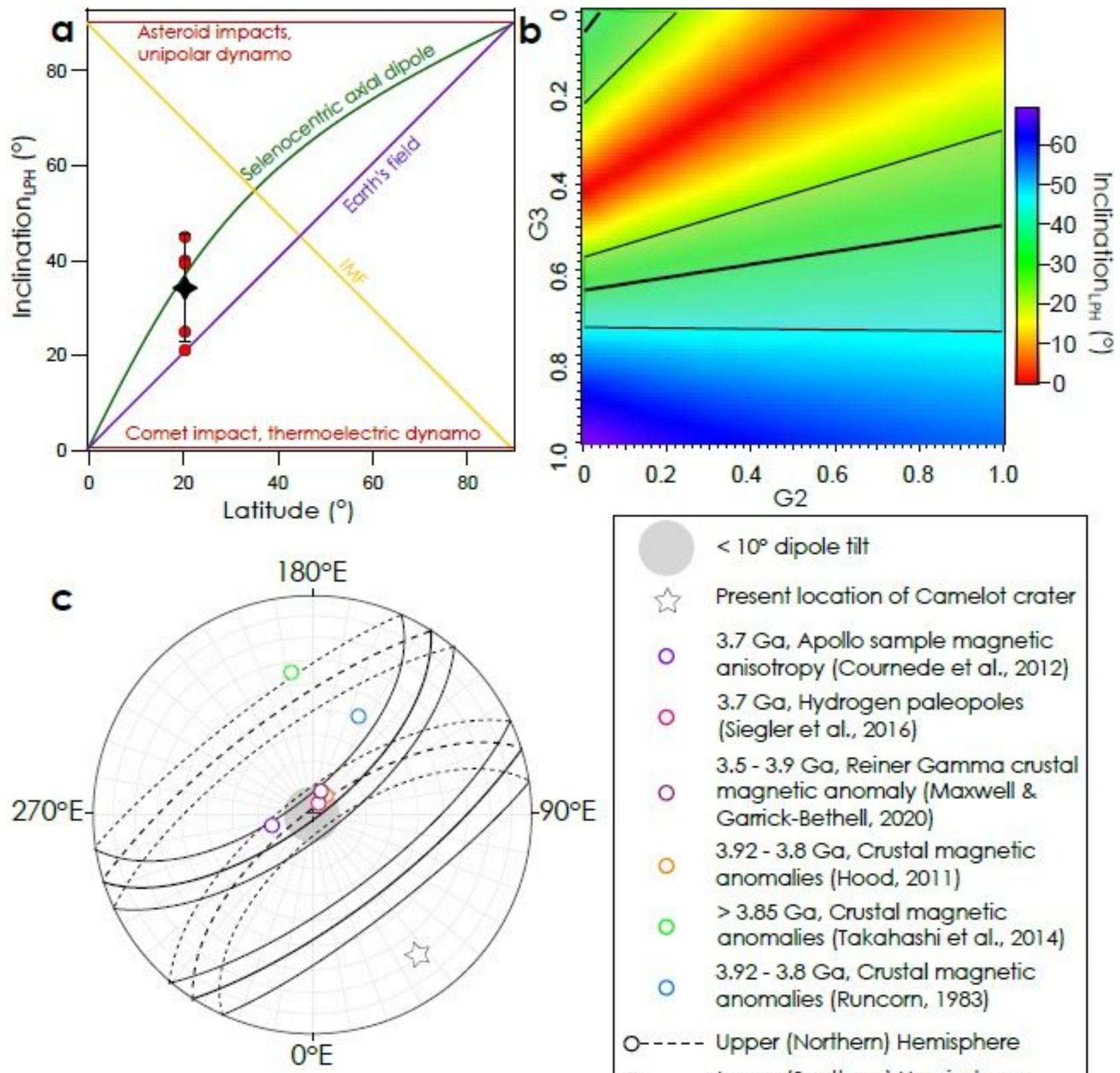

\begin{tabular}{|c|c|}
\hline & $<10^{\circ}$ dipole tilt \\
\hline$\hat{\imath}$ & Present location of Camelot crater \\
\hline 0 & $\begin{array}{l}3.7 \mathrm{Ga} \text {, Apollo sample magnetic } \\
\text { anisotropy (Cournede et al., 2012) }\end{array}$ \\
\hline 0 & $\begin{array}{l}3.7 \mathrm{Ga} \text {, Hydrogen paleopoles } \\
\text { (Siegler et al., 2016) }\end{array}$ \\
\hline 0 & $\begin{array}{l}3.5 \text { - } 3.9 \text { Ga, Reiner Gamma crustal } \\
\text { magnetic anomaly (Maxwell \& } \\
\text { Garick-Bethell, 2020) }\end{array}$ \\
\hline 0 & $\begin{array}{l}3.92-3.8 \text { Ga, Crustal magnetic } \\
\text { anomalies (Hood, 2011) }\end{array}$ \\
\hline 0 & $\begin{array}{l}>3.85 \mathrm{Ga} \text {, Crustal magnetic } \\
\text { anomalies (Takahashi et al., 2014) }\end{array}$ \\
\hline O & $\begin{array}{l}3.92-3.8 \text { Ga, Crustal magnetic } \\
\text { anomalies (Runcorn, 1983) }\end{array}$ \\
\hline 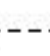 & Upper (Northern) Hemisphere \\
\hline & Lower (Southern) Hemisphere \\
\hline
\end{tabular}

\section{Figure 4}

Predictions for the paleoinclination of the lunar field, and implications for multipolarity and true polar wander. a, Paleoinclination results are shown at the present latitude of Camelot crater by the black symbol with $95 \%$ confidence error bars. Red dots are the measured paleoinclinations from each specimen of 75055. Predicted inclination versus latitude curves (see Figure 1) are shown for impact fields, a unipolar dynamo and a thermoelectric dynamo (red lines), Earth's magnetic field (purple line), the IMF 
(yellow line), and a selenocentric axial dipole (green line). b, The predicted magnetic inclination at Camelot crater for a magnetic field with zonal dipolar, quadrupolar and octupolar components, where G2 and G3 are the quadrupolar to dipolar and octupolar to dipolar field ratios, respectively. The thick black line is the mean paleoinclination and the regions bounded by thin black lines represent the $95 \%$ confidence interval. Both low and high degrees of multipolarity are permitted by our measured paleoinclination. c, Equal area stereographic projection showing the possible paleopole locations $3.7 \mathrm{Ga}$ ago in present-day lunar geographic coordinates. Open symbols/dashed lines and closed symbols/solid lines are in the northern and southern hemisphere, respectively. The star is the current location of Camelot crater where the samples were collected. The thick black lines are the permitted locations of the north pole calculated from our mean recovered paleoinclination. Thin black lines are the $95 \%$ confidence interval on our measurement. The grey shaded region represents $<10$ dipole tilt. North 3.7 Ga paleopole locations from independent studies are shown in purple5, pink7, red52, orange6, green51 and blue55. 